\title{
Families of Motives and the Mumford-Tate Conjecture
}

\author{
Ben Moonen
}

\begin{abstract}
We give an overview of some results and techniques related to the Mumford-Tate conjecture for motives over finitely generated fields of characteristic 0. In particular, we explain how working in families can lead to non-trivial results.
\end{abstract}

Mathematics Subject Classification (2010). Primary 14C30, 14F20; Secondary $14 \mathrm{D}$.

Keywords. Hodge theory, Galois representations, Motives.

\section{Introduction}

The goal of this article is to give an account of some results and techniques in the study of algebraic cycles, with special focus on the Mumford-Tate conjecture. This conjecture was stated in Mumford's paper [60] from 1966, in which he reported on joint work with Tate, introducing, in particular, what we now call the (special) Mumford-Tate group of an abelian variety. Not long before, Serre [75] had begun the study of Galois representations on the Tate modules $T_{\ell}$ of abelian varieties over a number fields, and had introduced the Lie algebra $\mathfrak{g}_{\ell} \subset \mathfrak{g l}\left(T_{\ell} \otimes \mathbb{Q}_{\ell}\right)$ of the image of such a representation. Mumford's paper ends with the conjecture that this Lie algebra, or more precisely, its intersection with $\mathfrak{s l}\left(T_{\ell} \otimes \mathbb{Q}_{\ell}\right)$, should be equal to the Lie algebra of the special Mumford-Tate group tensored with $\mathbb{Q}_{\ell}$. In a more tentative form ("on peut même espérer que...") the same problem is stated in Serre's Résumé des cours de 1965-1966 [76].

The MTC (Mumford-Tate Conjecture) is nowadays formulated in much greater generality and has no special relation to abelian varieties, other than that the strongest known results are about abelian motives. The context for it is that we consider a complete nonsingular variety $X$ (or more generally a motive) over a finitely generated field $K$ of characteristic 0 . If we choose a complex embedding $\sigma: K \rightarrow \mathbb{C}$, we may consider the singular ("Betti') cohomology $\mathrm{H}_{\sigma}=\mathrm{H}_{\mathrm{B}}\left(X_{\sigma}, \mathbb{Q}\right)$ of $X_{\sigma}$, which carries a Hodge structure. On the other hand, for $\ell$ a prime number, we have an 
action of $\operatorname{Gal}(\bar{K} / K)$ on the $\ell$-adic cohomology $\mathrm{H}_{\ell}=\mathrm{H}_{\text {ét }}\left(X_{\bar{K}}, \mathbb{Q}_{\ell}\right)$. The MTC then takes the form of a conjectural equality

$$
\operatorname{MT}\left(X_{\sigma}\right) \otimes \mathbb{Q}_{\ell} \stackrel{?}{=} G_{\ell}^{0}(X)
$$

of algebraic groups. Here $\mathrm{MT}\left(X_{\sigma}\right) \subset \mathrm{GL}\left(\mathrm{H}_{\sigma}\right)$ is the Mumford-Tate group, which is a reductive group over $\mathbb{Q}$ that in a suitable sense controls the Hodge structure $\mathrm{H}_{\sigma}$, and $G_{\ell}^{0}(X) \subset \mathrm{GL}\left(\mathrm{H}_{\ell}\right)$ is (the identity component of) the Zariski closure of the image of the Galois representation $\rho_{\ell}: \operatorname{Gal}(\bar{K} / K) \rightarrow \mathrm{GL}\left(\mathrm{H}_{\ell}\right)$, which is an algebraic group over $\mathbb{Q}_{\ell}$.

The MTC derives its plausibility less from overwhelming evidence than from its natural place in a web of conjectures, and in particular from its role as a bridge between the Hodge Conjecture (HC) and the Tate Conjecture (TC). If the $\mathrm{HC}$ and TC are both true (for all complete nonsingular varieties), so is the MTC. The main point here is that a reductive algebraic group is completely characterized by its tensor invariants. For the Mumford-Tate group these tensor invariants are the Hodge classes; for the $\ell$-adic algebraic Galois group $G_{\ell}^{0}$ these are the Tate classes. (The groups $G_{\ell}^{0}$ are not known to be reductive in general; that they are is part of the Tate conjecture. See however Remark 2.2.6(iv).) According to the HC and TC, the Hodge (resp. Tate) classes should be precisely the cohomology classes of algebraic cycles; assuming both conjectures, it follows that $\mathrm{MT}\left(X_{\sigma}\right) \otimes \mathbb{Q}_{\ell}$ and $G_{\ell}^{0}(X)$ are equal because they have the same tensor invariants.

This reasoning does not give a direct comparison between $\operatorname{MT}\left(X_{\sigma}\right)$ and $G_{\ell}^{0}(X)$. Rather, it tells us that there should be a third group to which both are equal: on both sides the invariants are conjecturally the cohomology classes of algebraic cycles. The natural habitat for this third object is the theory of motives. It is not clear, however, why there should be a reductive algebraic group whose tensor invariants are precisely the cohomology classes of algebraic cycles. In the language of tensor categories this means we should like to have a category of (pure) motives that is a semisimple Tannakian category. If one tries to build such a category, one is confronted with a lack of construction techniques for algebraic cycles, and in particular with the fact that Grothendieck's Standard Conjecture B ("of Lefschetz type") is not known. In the past decades, some very satisfactory workarounds have been introduced, such as Deligne's category of motives for absolute Hodge cycles [34], [37] (extended to mixed motives by Jannsen [47]) and André's category of motives for motivated cycles [5] (which is known [8] to be equivalent to the "pure" part of Nori's category of motives; see [44]). In this article we will use André's theory, though we would get essentially the same results by working with motives for absolute Hodge classes. To a motive $\mathbf{M}$ (e.g., a complete nonsingular variety) we can then associate a motivic Galois group $G_{\mathrm{mot}}(\mathbf{M})$ whose tensor invariants are the motivated cycles. Even if we do not know if all motivated cycles are algebraic cycle classes, they form a perfectly good substitute in the context of the MTC. The natural, "motivic", form of the MTC is then that $\operatorname{MT}\left(X_{\sigma}\right) \otimes \mathbb{Q}_{\ell}$ and $G_{\ell}^{0}(X)$ should both be equal to the motivic Galois group. 
Sections 2 and 3 of this article are of an expository nature. In Section 2 we introduce Mumford-Tate groups and explain their role from several perspectives. Furthermore, we introduce their $\ell$-adic counterparts $G_{\ell}^{0}$, and we discuss the Mumford-Tate conjecture. In Section 3 we introduce pure motives in the sense of André, as well as their motivic Galois groups, and we discuss how this leads to a refined, "motivic", version of the Mumford-Tate conjecture.

At the end of each section we present an overview of some known results. The author felt it was not an easy task to summarize decades of research in a couple of pages, and can only hope he has managed to give a reasonably accurate picture of the main results, without too many serious omissions. In any case we have included many references to the literature, where the reader may find further details. What remains somewhat underexposed in this article is the role of the theory of compatible systems of $\ell$-adic representations. For this, and many other aspects that are excluded here, Serre's beautiful papers [79] and [84] form a good starting point.

Of course we want to get further than conjectures, and this is where one of the main themes of this article comes in. Namely, in Section 4 we discuss how working with families of varieties provides us with additional tools that in some cases lead to very nontrivial results. It will come as no surprise that monodromy is among these tools. What perhaps is surprising is that, even though this notion has been omnipresent in geometry since the 19th century, in recent years people have found still better ways to exploit monodromy; as we will see, it lies at the heart of some recent developments. In the study of families of varieties, we will in particular discuss how the Mumford-Tate groups, $\ell$-adic algebraic Galois groups and motivic Galois groups of the fibres vary. Though the variation is of an erratic nature, there is now a reasonably good understanding of the "jump loci", and it is nice to see, in Theorem 4.3.1 and Corollary 4.3.9 for instance, how monodromy plays a unifying role.

Though much of what we discuss in this section is based on work of other people, and in particular on the theory developed by André in [5], we hope that our presentation will make these results more accessible, and will in particular clarify the mutual relationship between motives and their Hodge and $\ell$-adic realizations. As an illustration of the power of these techniques, we end this section with a quick proof of the Tate conjecture for divisor classes on algebraic surfaces in some particular families.

The geometer may complain about our strong focus on abstract notions such as Tannakian categories and properties of algebraic groups. Indeed, many results on which we report are based on a good understanding of formal structures as much as on geometry. What geometric intuition is there behind the fact that the Hodge conjecture and the Tate conjecture are "trivially" true ${ }^{1}$ for simple abelian varieties of prime dimension, whereas already for simple abelian fourfolds it is a deep open

\footnotetext{
${ }^{1}$ in the sense that all Hodge classes, resp. Tate classes, are polynomials in divisor classes-what goes into the proof is of course by no means trivial!
} 
problem? To the author's mind, the fact that conjectures about algebraic cycles have a direct relation to representation theory of reductive groups is one of the wonders of the subject.

Notation and conventions. (a) Many of the categories we consider involve a base field or base variety (often called $K$ or $S$ ), and a coefficient ring or field Q. As a general rule, the base field or variety is given in parenthesis and the coefficient field as a subscript. Example: $\operatorname{Mot}(K)_{\mathrm{Q}}$ is the category of motives over $K$ with coefficients in $Q$.

(b) For us, an algebraic group $G$ over a field $K$ is a special case of a group scheme over $K$. It is therefore understood that by a homomorphism $G_{1} \rightarrow G_{2}$ between such algebraic groups we mean a homomorphism over $K$, and by a representation of $G$ we mean a (finite dimensional) representation on a $K$-vector space. The category of such representations is denoted by $\operatorname{Rep}(G)$.

\section{The Mumford-Tate conjecture}

\subsection{Mumford-Tate groups}

We start by reviewing some abstract aspects of Hodge theory. Later in this section we will discuss how this is relevant to Algebraic Geometry.

Pure Hodge structures. Let $\mathrm{HS}_{\mathbb{Q}}$ be the category of pure $\mathbb{Q}$-Hodge structures. (In what follows we shall mostly work with $\mathbb{Q}$-coefficients.) By definition, an object of $\mathrm{HS}_{\mathbb{Q}}$ is a finite dimensional graded vector space $H=\oplus_{n \in \mathbb{Z}} H^{(n)}$ such that each $H^{(n)}$ is given a Hodge structure of weight $n$. This category $\mathrm{HS}_{\mathbb{Q}}$ is a neutral Tannakian category; in particular we have direct sums, tensor products and duals; on the underlying $\mathbb{Q}$-vector spaces they are given by the usual constructions. In addition we have Tate twists: $\mathbb{Q}(n)$ is the 1 -dimensional $\mathbb{Q}$-vector space $(2 \pi i)^{n} \cdot \mathbb{Q}$ with Hodge structure purely of type $(-n,-n)$, and if $H$ is a Hodge structure then we write $H(n)$ for $H \otimes \mathbb{Q}(n)$. Note that $H \mapsto H(n)$ decreases the weight by $2 n$.

If $H$ is a pure $\mathbb{Q}$-Hodge structure of weight $n$, a polarization of $H$ is a morphism of Hodge structures $\phi: H \otimes H \rightarrow \mathbb{Q}(-n)$ that satisfies a certain positivity property. We refer to [31], Définition 2.1.15 or [66], Section 2.1.2 for the precise definition. What matters for us is that the subcategory $\mathrm{HS}_{\mathbb{Q}}^{\text {pol }} \subset \mathrm{HS}_{\mathbb{Q}}$ of polarizable $\mathbb{Q}$-Hodge structures (those which admit a polarization) is semisimple, and that the Hodge structures that are of interest for us all lie in this subcategory. The subcategory $\mathrm{HS}_{\mathbb{Q}}^{\text {pol }}$ is closed under direct sums, tensor products and duality, and every subquotient of a polarizable Hodge structure is itself again polarizable.

If $H$ is a pure polarizable $\mathbb{Q}$-Hodge structure, its endomorphism algebra $D=$ $\operatorname{End}_{\mathrm{HS}_{\mathbb{Q}}}(H)$ is a finite dimensional semisimple $\mathbb{Q}$-algebra. The choice of a polarization $\phi$ gives rise to an involution $d \mapsto d^{*}$ on $D$, and it can be shown that this is a positive involution. The pair $(D, *)$ is therefore of the type classified by Albert; we refer to [62], Chapter 21, for further details on this classification. Let us only record here that the centre of $D$ is either a totally real field or a CM field. 
The Deligne torus. Let $\mathbb{S}$ be the algebraic torus over $\mathbb{R}$ obtained as $\mathbb{S}=\operatorname{Res}_{\mathbb{C} / \mathbb{R}}\left(\mathbb{G}_{\mathrm{m}}\right)$, where Res denotes restriction of scalars ("Weil restriction"). We have $\mathbb{S}(\mathbb{R})=\mathbb{C}^{\times}$ and $\mathbb{S}(\mathbb{C})=\mathbb{C}^{\times} \times \mathbb{C}^{\times}$. The character group of $\mathbb{S}$ is given by $X^{*}(\mathbb{S})=\mathbb{Z} \cdot z \oplus \mathbb{Z} \cdot \bar{z}$, with complex conjugation acting by $z \leftrightarrow \bar{z}$. Define $\mathrm{Nm}=z \bar{z}: \mathbb{S} \rightarrow \mathbb{G}_{\mathrm{m}, \mathbb{R}} ;$ on $\mathbb{R}$ valued points it is given by the usual norm map $\mathbb{C}^{\times} \rightarrow \mathbb{R}^{\times}$. Let $w: \mathbb{G}_{\mathrm{m}, \mathbb{R}} \rightarrow \mathbb{S}$ be the cocharacter given on $\mathbb{R}$-valued points by the inclusion $\mathbb{R}^{\times} \hookrightarrow \mathbb{C}^{\times}$; so $z \circ w$ and $\bar{z} \circ w$ are both the identity on $\mathbb{G}_{\mathrm{m}, \mathbb{R}}$.

To give a representation $\mathbb{S} \rightarrow \mathrm{GL}(V)$, for $V$ a real vector space, is the same as giving a decomposition $V_{\mathbb{C}}=\oplus_{p, q} V_{\mathbb{C}}^{p, q}$ with the property that $\overline{V_{\mathbb{C}}^{p, q}}=V_{\mathbb{C}}^{q, p}$. In this correspondence, $V_{\mathbb{C}}^{p, q}$ is the subspace of elements $v \in V_{\mathbb{C}}$ on which $\left(z_{1}, z_{2}\right) \in$ $\mathbb{C}^{\times} \times \mathbb{C}^{\times}=\mathbb{S}(\mathbb{C})$ acts as multiplication by $z_{1}^{-p} z_{2}^{-q}$. (The minus signs in the exponents are just a convention, which we will not try to justify here.) Therefore, a $\mathbb{Q}$-Hodge structure $H$ of weight $n$ may be described as a finite dimensional $\mathbb{Q}$-vector space, together with a representation $h: \mathbb{S} \rightarrow \mathrm{GL}(H)_{\mathbb{R}}$ such that $h \circ w: \mathbb{G}_{\mathrm{m}} \rightarrow \mathrm{GL}(H)_{\mathbb{R}}$ is given by $z \mapsto z^{-n} \cdot \mathrm{id}_{H}$. To describe an arbitrary Hodge structure (a sum of pieces that are pure of some weight $n$ ), we consider a $\mathbb{Q}$-vector space $H$ with a representation $h$ as before, such that $h \circ w$ is defined over $\mathbb{Q}$; in that case the cocharacter $h \circ w: \mathbb{G}_{\mathrm{m}, \mathbb{Q}} \rightarrow$ $\mathrm{GL}(H)$ gives rise to a decomposition $H=\oplus H^{(n)}$ such that $z \in \mathbb{Q}^{\times}$acts on $H^{(n)}$ as multiplication by $z^{-n}$, and on each $H^{(n)}$ we have a Hodge structure of weight $n$.

Example 2.1.1. The Hodge structure $\mathbb{Q}(n)$ is given by $\mathrm{Nm}^{n}: \mathbb{S} \rightarrow \mathbb{G}_{\mathrm{m}}$. If $H$ and $H^{\prime}$ are $\mathbb{Q}$-Hodge structures given by homomorphisms $h: \mathbb{S} \rightarrow \operatorname{GL}(H)_{\mathbb{R}}$ and $h^{\prime}: \mathbb{S} \rightarrow$ $\mathrm{GL}\left(H^{\prime}\right)_{\mathbb{R}}$, the tensor product $H \otimes H^{\prime}$ is given by the homomorphism $h \otimes h^{\prime}$. It follows that $H(n)$ is given by $\mathrm{Nm}^{n} \cdot h$.

Definition 2.1.2. Let $H$ be a $\mathbb{Q}$-Hodge structure, and let $h: \mathbb{S} \rightarrow \operatorname{GL}(H)_{\mathbb{R}}$ be the homomorphism that gives the Hodge structure. Then the Mumford-Tate group of $H$, notation $\operatorname{MT}(H)$, is the smallest algebraic subgroup $M \subset \mathrm{GL}(H)$ over $\mathbb{Q}$ such that $h$ factors through the subgroup $M_{\mathbb{R}} \subset \mathrm{GL}(H)_{\mathbb{R}}$.

The most important property of the Mumford-Tate group is the following.

Key Property 2.1.3. Let $H$ be a $\mathbb{Q}$-Hodge structure. For $r, s \in \mathbb{N}$, define $T^{r, s}=$ $T^{r, s}(H)=H^{\otimes r} \otimes\left(H^{\vee}\right)^{\otimes s}$. Let $T$ be any space of the form $T^{r_{1}, s_{1}} \oplus \cdots \oplus T^{r_{k}, s_{k}}$, and let $V \subset T$ be a $\mathbb{Q}$-subspace. Then $V$ is stable under the action of $\operatorname{MT}(H)$ on $T$ if and only if $V$ is a sub-Hodge structure of $T$.

We give some further basic properties. We focus on the polarizable case.

Properties 2.1.4. Let $H$ be a polarizable $\mathbb{Q}$-Hodge structure.

(i) The Mumford-Tate group $\mathrm{MT}(H)$ is a connected reductive subgroup of $\mathrm{GL}(H)$. (If $H$ is not polarizable then $\operatorname{MT}(H)$ is not reductive, in general.)

(ii) Suppose $H$ is pure of weight $n$. If $n=0$ then $\operatorname{MT}(H)$ is contained in $\operatorname{SL}(H)$. If $n \neq 0$, the homotheties $\mathbb{G}_{\mathrm{m}} \cdot \mathrm{id}_{H}$ are contained in $\operatorname{MT}(H)$.

(iii) If $H_{1}$ and $H_{2}$ are $\mathbb{Q}$-Hodge structures then $\mathrm{MT}\left(H_{1} \oplus H_{2}\right)$ is an algebraic subgroup of $\operatorname{MT}\left(H_{1}\right) \times \operatorname{MT}\left(H_{2}\right)$ and the two projections $\operatorname{MT}\left(H_{1} \oplus H_{2}\right) \rightarrow \operatorname{MT}\left(H_{i}\right)$ are surjective. 
(iv) For $H_{1}, \ldots, H_{r}$ in $\mathrm{HS}_{\mathbb{Q}}$ and positive integers $m_{1}, \ldots, m_{r}$ we have

$$
\operatorname{MT}\left(H_{1}^{m_{1}} \oplus \cdots \oplus H_{r}^{m_{r}}\right) \cong \operatorname{MT}\left(H_{1} \oplus \cdots \oplus H_{r}\right) .
$$

As a special case of this, for $H$ in $\mathrm{HS}_{\mathbb{Q}}$ and $r \geq 1$, the Mumford-Tate group of $H^{\oplus r}$ is isomorphic to $\mathrm{MT}(H)$, acting diagonally on $H^{\oplus r}$.

Remark 2.1.5. Let $\mu_{\mathbb{S}}: \mathbb{G}_{\mathrm{m}, \mathbb{C}} \rightarrow \mathbb{S}_{\mathbb{C}}$ be the cocharacter such that $z \circ \mu_{\mathbb{S}}$ is the identity on $\mathbb{G}_{\mathrm{m}, \mathbb{C}}$ and $\bar{z} \circ \mu_{\mathbb{S}}$ is the trivial endomorphism of $\mathbb{G}_{\mathrm{m}, \mathbb{C}}$. On $\mathbb{C}$-valued points $\mu_{\mathbb{S}}$ is the homomorphism $\mathbb{C}^{\times} \rightarrow\left(\mathbb{C}^{\times} \times \mathbb{C}^{\times}\right)$given by $a \mapsto(a, 1)$.

Let $H$ be a Hodge structure given by a homomorphism $h: \mathbb{S} \rightarrow \operatorname{GL}(H)_{\mathbb{R}}$. Write $\mu=h_{\mathbb{C}} \circ \mu_{\mathbb{S}}: \mathbb{G}_{\mathrm{m}, \mathbb{C}} \rightarrow \mathrm{GL}(H)_{\mathbb{C}}$. It is not hard to show that $\mathrm{MT}(H)$ is the smallest algebraic subgroup of $\mathrm{GL}(H)$ such that the cocharacter $\mu$ factors through $\operatorname{MT}(H)_{\mathbb{C}}$. Note that $z \in \mathbb{C}^{\times}$acts on $H^{p, q}$ (via $\mu$ ) as multiplication by $z^{-p}$. In Section 2.4 we will further discuss how this leads to restrictions on the possibilities for the Mumford-Tate group.

It is important to also understand the Mumford-Tate group from a Tannakian perspective. If we denote by $\langle H\rangle \subset \mathrm{HS}_{\mathbb{Q}}$ the Tannakian subcategory generated by $H$, the forgetful functor $\omega:\langle H\rangle \rightarrow \operatorname{Vec}_{\mathbb{Q}}$ is a fibre functor that has $\operatorname{MT}(H)$ as its automorphism group. This means that $\langle H\rangle$ is equivalent to the category $\operatorname{Rep}(\mathrm{MT}(H))$ of (finite dimensional, algebraic) representations of $\mathrm{MT}(H)$. In view of property 2.1.4(i), this connects Hodge theory to the representation theory of reductive groups.

Definition 2.1.6. Let $H$ be a $\mathbb{Q}$-Hodge structure. An element $\xi \in H$ is called a Hodge class if $\xi$ is purely of type $(0,0)$ in the Hodge decomposition $H_{\mathbb{C}}=\oplus H_{\mathbb{C}}^{p, q}$.

Put differently, the Hodge classes are those rational classes (i.e., classes in the underlying $\mathbb{Q}$-vector space $H)$ that are purely of type $(0,0)$ in the Hodge decomposition of the complexification of $H$. Writing $\mathbf{1}=\mathbb{Q}(0)$, which is the identity object with respect to the tensor product in $\mathrm{HS}_{\mathbb{Q}}$, the space of Hodge classes in $H$ can be identified with $\operatorname{Hom}_{\mathrm{HS}}(\mathbf{1}, H)$.

Lemma 2.1.7. Let $H$ be a $\mathbb{Q}$-Hodge structure that is pure of weight $n$, and let $T=$ $T^{r_{1}, s_{1}} \oplus \cdots \oplus T^{r_{k}, s_{k}}$ be a tensor construction as in 2.1.3. Then an element $\xi \in T$ is a Hodge class in $T$ if and only if $\xi$ is invariant under the action of $\mathrm{MT}(H)$ on $T$.

Proof. Let $L \subset T \oplus \mathbb{Q}(0)=T \oplus T^{0,0}$ be the line spanned by $(\xi, 1)$. Then $L$ is a sub-Hodge structure of $T \oplus \mathbb{Q}(0)$ if and only if $\xi$ is a Hodge class. On the other hand, since $\operatorname{MT}(H)$ acts as the identity on $\mathbb{Q}(0)$, we see that $L \subset T \oplus \mathbb{Q}(0)$ is a sub-representation of $\mathrm{MT}(H)$ if and only if $\xi$ is invariant under $\mathrm{MT}(H)$. Now use 2.1.3.

Remark 2.1.8. If $H$ is a vector space over a field $k$ of characteristic 0 and $G \subset \mathrm{GL}(H)$ is a reductive subgroup, then $G$ is completely determined by its tensor invariants; see [35], Proposition 3.1.(c). Hence the Mumford-Tate group of a polarizable $\mathbb{Q}$ Hodge structure $H$ may also, somewhat indirectly, be defined as the unique reductive subgroup of $\mathrm{GL}(H)$ whose tensor invariants are the Hodge classes. 
Example 2.1.9. Given $\mathbb{Q}$-Hodge structures $H_{1}$ and $H_{2}$, say of weights $n_{1}$ and $n_{2}$, consider the Hodge structure $\underline{\operatorname{Hom}}\left(H_{1}, H_{2}\right)=H_{1}^{\vee} \otimes H_{2}$, which has weight $n_{2}-n_{1}$. The Hodge classes in $\underline{\operatorname{Hom}}\left(H_{1}, H_{2}\right)$ are precisely the morphisms of $\mathbb{Q}$-Hodge structures $H_{1} \rightarrow H_{2}$; in other words

$$
\begin{aligned}
\operatorname{Hom}_{\mathrm{HS}_{\mathbb{Q}}}\left(H_{1}, H_{2}\right) & =\left\{\text { Hodge classes in } \underline{\operatorname{Hom}}\left(H_{1}, H_{2}\right)\right\} \\
& =\underline{\operatorname{Hom}}\left(H_{1}, H_{2}\right)^{\operatorname{MT}\left(H_{1} \oplus H_{2}\right)} .
\end{aligned}
$$

(Note that these spaces can be non-zero only if $n_{1}=n_{2}$.) As a special case of this, for $H$ in $\mathrm{HS}_{\mathbb{Q}}$ we have $\operatorname{End}_{\mathrm{HS}_{\mathbb{Q}}}(H)=\underline{\operatorname{End}}(H)^{\mathrm{MT}(H)}$.

Hodge structures on the cohomology of varieties. If $Y$ is a compact Kähler manifold, its singular cohomology $\mathrm{H}^{n}(Y, \mathbb{Q})$ carries a natural Hodge structure of weight $n$. We refer to [66] or [105] for a detailed discussion of how this is obtained. If $f: Y_{1} \rightarrow Y_{2}$ is a morphism of compact Kähler manifolds, the induced map $f^{*}: \mathrm{H}^{n}\left(Y_{2}, \mathbb{Q}\right) \rightarrow$ $\mathrm{H}^{n}\left(Y_{1}, \mathbb{Q}\right)$ is a morphism of Hodge structures. The Künneth isomorphism $\mathrm{H}^{\bullet}\left(Y_{1} \times\right.$ $\left.Y_{2}, \mathbb{Q}\right) \stackrel{\sim}{\longrightarrow} \mathrm{H}^{\bullet}\left(Y_{1}, \mathbb{Q}\right) \otimes \mathrm{H}^{\bullet}\left(Y_{2}, \mathbb{Q}\right)$ is an isomorphism of Hodge structures. Note that the Hodge structure $\mathrm{H}^{n}(Y, \mathbb{Q})$ is not, in general, polarizable.

If $X$ is a projective nonsingular variety over $\mathbb{C}$, the associated complex analytic variety $X^{\text {an }}$ is compact Kähler and we have a natural Hodge structure of weight $n$ on the singular cohomology group $\mathrm{H}^{n}=\mathrm{H}^{n}\left(X^{\mathrm{an}}, \mathbb{Q}\right)$. In this case we do get that $\mathrm{H}^{n}$ is polarizable. We can extend this to complete nonsingular varieties $X$, even though $X^{\text {an }}$ need not be Kähler. Indeed, by Chow's lemma and resolution of singularities we can find a surjective morphism $f: Y \rightarrow X$ with $Y$ projective nonsingular. The induced map $f^{*}: \mathrm{H}^{n}(X, \mathbb{Q}) \rightarrow \mathrm{H}^{n}(Y, \mathbb{Q})$ is injective, realizing $\mathrm{H}^{n}(X, \mathbb{Q})$ as a subHodge structure (automatically polarizable) of $\mathrm{H}^{n}(Y, \mathbb{Q})$, and the Hodge structure on $\mathrm{H}^{n}(X, \mathbb{Q})$ thus obtained is independent of choices.

Many examples that we are going to discuss use the fact that the functor that sends a complex abelian variety $X$ to the $\mathbb{Q}$-Hodge structure $\mathrm{H}_{1}(X, \mathbb{Q}$ ) (which is the dual of $\left.\mathrm{H}^{1}(X, \mathbb{Q})\right)$ is an equivalence of categories

$$
\left(\begin{array}{c}
\text { complex abelian varieties } \\
\text { up to isogeny }
\end{array}\right) \rightarrow\left(\begin{array}{c}
\text { polarizable } \mathbb{Q} \text {-Hodge structures } \\
\text { of type }(-1,0)+(0,-1)
\end{array}\right) .
$$

(There is also a version with $\mathbb{Z}$-coefficients which gives an equivalence of categories between abelian varieties and integral polarizable Hodge structures of type $(-1,0)+$ $(0,-1)$.) We define the Mumford-Tate group of an abelian variety $X$ by $\operatorname{MT}(X)=$ $\operatorname{MT}(\mathrm{H})$, where $\mathrm{H}=\mathrm{H}_{1}(X, \mathbb{Q})$. Writing $\operatorname{End}^{0}(X)=\operatorname{End}(X) \otimes \mathbb{Q}$, Example 2.1.9 gives

$$
\operatorname{End}^{0}(X) \stackrel{\sim}{\longrightarrow} \operatorname{End}_{\mathrm{HS}_{\mathbb{Q}}}(\mathrm{H})=\underline{\operatorname{End}}(\mathrm{H})^{\mathrm{MT}(X)} .
$$

Example 2.1.10. A polarizable $\mathbb{Q}$-Hodge structure $H$ is said to be of $C M$ type if its Mumford-Tate group $\operatorname{MT}(H)$ is a torus. It can be shown that this is equivalent to the condition that the endomorphism algebra $\operatorname{End}_{\mathrm{HS}_{\mathbb{Q}}}(H)$ contains a commutative semisimple $\mathbb{Q}$-algebra $F$ such that $H$ is free of rank 1 as an $F$-module.

If $H$ is simple then it is of CM type if and only if its endomorphism algebra $E=\operatorname{End}_{\mathrm{HS}_{\mathbb{Q}}}(H)$ is a field of $\operatorname{degree} \operatorname{dim}(H)$ over $\mathbb{Q}$. It can be shown that either 
$H \cong \mathbb{Q}(n)$ for some $n$, in which case $E=\mathbb{Q}$, or else $E$ is a CM field. In general, we can decompose a polarizable $\mathbb{Q}$-Hodge structure $H$ as $H=H_{1}^{m_{1}} \oplus \cdots \oplus H_{r}^{m_{r}}$ with $H_{1}, \ldots, H_{r}$ simple and pairwise non-isomorphic. Then $H$ is of CM type if and only if each $H_{i}$ is of CM type.

Suppose $H$ is simple of CM type, of weight $n$. Let $\Sigma$ be the set of complex embeddings of its endomorphism algebra $E$. For $\sigma \in \Sigma$, let $H_{\mathbb{C}}(\sigma) \subset H_{\mathbb{C}}$ be the subspace on which $E$ acts through the embedding $\sigma$. Writing $H_{\mathbb{C}}^{p, q}(\sigma)=H_{\mathbb{C}}(\sigma) \cap H_{\mathbb{C}}^{p, q}$ we then have a decomposition

$$
H_{\mathbb{C}}=\bigoplus_{\sigma \in \Sigma} \bigoplus_{p+q=n} H_{\mathbb{C}}^{p, q}(\sigma) \quad \text { with } \quad \overline{H_{\mathbb{C}}^{p, q}(\sigma)}=H_{\mathbb{C}}^{q, p}(\bar{\sigma}) .
$$

Each $H_{\mathbb{C}}(\sigma)$ is 1-dimensional, so for $\sigma \in \Sigma$ there is a unique integer $p=p(\sigma)$ with $H_{\mathbb{C}}^{p, q}(\sigma) \neq 0$. This gives us a function $p: \Sigma \rightarrow \mathbb{Z}$ with $p(\bar{\sigma})=n-p(\sigma)$. The Hodge structure $H$ is completely determined by the pair $(E, p)$.

A classical example where this arises is when we have a $g$-dimensional complex abelian variety $X$ of CM type, which is equivalent to saying that the Hodge structure $\mathrm{H}=\mathrm{H}_{1}(X, \mathbb{Q})$ is of CM type. For simplicity, assume $X$ is simple, so that $E=$ $\operatorname{End}^{0}(X)=\operatorname{End}_{\mathrm{HS}}(\mathrm{H})$ is a CM field of degree $2 g$. In this case the function $p: \Sigma \rightarrow \mathbb{Z}$ only takes the values -1 and 0 . Instead of giving the function $p$ we may give the subset $\Phi \subset \Sigma$ of embeddings $\sigma$ for which $p(\sigma)=-1$; this set has the property that $\Sigma=\Phi \amalg \bar{\Phi}$. The pair $(E, \Phi)$ is classically called the CM type of $X$. For later use let us make explicit how we can recover $\mathrm{H}$, and therefore also $X$ up to isogeny, from $(E, \Phi)$ : As underlying $\mathbb{Q}$-vector space we take $\mathrm{H}=E$; then $\mathrm{H}_{\mathbb{C}}=\oplus_{\sigma \in \Sigma} \mathbb{C}^{(\sigma)}$ (in which the superscript $(\sigma)$ is included only for bookkeeping purposes), and we declare the summand $\mathbb{C}^{(\sigma)}$ to be of Hodge type $(-1,0)$ (resp. $\left.(0,-1)\right)$ if $\sigma \in \Phi(\operatorname{resp} . \sigma \notin \Phi)$.

It is easy to see that the Tannakian subcategory of $\mathrm{HS}_{\mathbb{Q}}^{\text {pol }}$ consisting of $\mathrm{CM}$ Hodge structures is generated by the Hodge structures $\mathrm{H}_{1}(X, \mathbb{Q})$ associated with CM types $(E, \Phi)$. See for instance [2], Section 2 .

Cycle classes. If $X$ is a proper smooth algebraic variety over $\mathbb{C}$ then to an algebraic cycle $Z$ of codimension $n$ we can attach a cohomology class $\operatorname{cl}(Z) \in \mathrm{H}^{2 n}(X, \mathbb{Q}(n))$, which is a Hodge class. The cohomology class that we obtain only depends on the class of $Z$ modulo rational equivalence, so we obtain a map

$$
c l: \mathrm{CH}^{n}(X) \otimes \mathbb{Q} \rightarrow\left\{\text { Hodge classes in } \mathrm{H}^{2 n}(X, \mathbb{Q}(n))\right\} .
$$

The Hodge conjecture expresses that all Hodge classes in the cohomology should arise from algebraic cycles in this way:

Conjecture 2.1.11 (The Hodge Conjecture). Let $X$ be a complete nonsingular complex algebraic variety. Then for every $n \geq 0$ the cycle class map (2.2) is surjective.

For $n=1$ the Hodge conjecture is true; this is Lefschetz's theorem on divisor classes. For $n=0$ and $n=d=\operatorname{dim}(X)$ the Hodge conjecture is "trivially" true, as $\mathrm{H}^{0}(X, \mathbb{Q}) \cong \mathbb{Q}(0)$ and $\mathrm{H}^{2 d}(X, \mathbb{Q}(d))$ are both 1-dimensional, spanned by the class of $X$ (assumed to be irreducible), and the class of a point on $X$, respectively. Apart from these cases, the Hodge conjecture is widely open. For further reading we recommend [50], [87], [103] and [106]. 
Remark 2.1.12 (The special Mumford-Tate group). In older literature, Hodge classes in a $\mathbb{Q}$-Hodge structure $H$ of even weight $2 p$ are often defined as the rational classes $\xi \in H$ that are purely of type $(p, p)$ in the Hodge decomposition. From a theoretical perspective it is more natural to replace $H$ by $H(p)$, in which case such " $(p, p)$ classes" become Hodge classes in the sense of Definition 2.1.6. For practical purposes it makes no big difference which version we use. Note, however, that $(p, p)$-classes with $p \neq 0$ are not detected as invariants under $\operatorname{MT}(H)$, as $\operatorname{MT}(H)$ contains the homotheties $z \cdot \operatorname{id}_{H}$, and these act on $H$ as multiplication by $z^{-2 p}$.

To remedy this, one may consider the special Mumford-Tate group $\operatorname{SMT}(H) \subset$ $\mathrm{SL}(H)$ (also sometimes called the Hodge group). To define it, consider the circle group $\mathbb{S}^{1}=\operatorname{Ker}\left(\mathrm{Nm}: \mathbb{S} \rightarrow \mathbb{G}_{\mathrm{m}, \mathbb{R}}\right)$. Then $\operatorname{SMT}(H)$ is defined as the smallest algebraic subgroup $M \subset \mathrm{GL}(H)$ such that $\left.h\right|_{\mathbb{S}^{1}}$ factors through $M_{\mathbb{R}}$. With notation as in 2.1.3, an element $t \in T^{r, s}$ is a rational $(q, q)$-class, with $q=p(r-s)$, if and only if $t$ is invariant under the action of $\operatorname{SMT}(H)$. This breaks down if we consider classes $t$ in an arbitrary tensor construction $T=T^{r_{1}, s_{1}} \oplus \cdots \oplus T^{r_{k}, s_{k}}$. The problem is that the special Mumford-Tate group does not see the weight of a Hodge structure. For instance, if $\xi \in H$ is a $(q, q)$-class with $q \neq 0$ then $(\xi, 1) \in H \oplus \mathbb{Q}(0)$ is invariant under the action of $\operatorname{SMT}(H)$ but is not a $(p, p)$-class.

If $H$ has weight 0 we have $\operatorname{SMT}(H)=\mathrm{MT}(H)$. If $H$ is pure of weight $n \neq 0$, we have $\operatorname{MT}(H)=\left(\mathbb{G}_{\mathrm{m}} \cdot \operatorname{id}_{H}\right) \cdot \operatorname{SMT}(H)$.

If $X$ is complete nonsingular, the rational $(p, p)$-classes in $\mathrm{H}^{\bullet}(X, \mathbb{Q})$ form a subring $\mathscr{B}(X)$, called the Hodge ring of $X$. If this Hodge ring is generated by divisor classes, it follows from Lefschetz theorem on $(1,1)$-classes that the Hodge conjecture for $X$ is true.

Example 2.1.13 (How to use the Mumford-Tate group). The Mumford-Tate group is particularly effective as a tool to study Hodge classes on abelian varieties. For instance, it leads to the striking fact that we know the Hodge conjecture to be true for all simple abelian varieties of prime dimension; see [70]. (By contrast, already for abelian fourfolds the Hodge conjecture is still open.)

For a given dimension $g=\operatorname{dim}(X)$ there is a finite list of possible types for $\operatorname{End}^{0}(X)$ (see [62], Section 21). This leads to the following strategy. As before, $X$ is an abelian variety and $\mathrm{H}=\mathrm{H}_{1}(X, \mathbb{Q})$.

(a) Given $g=\operatorname{dim}(X)$ and $\operatorname{End}^{0}(X)$, try to decide what $\operatorname{MT}(X) \subset \mathrm{GL}(\mathrm{H})$ is, based on the properties listed in 2.1.4 together with (2.1).

(b) If successful, determine the Hodge ring $\mathscr{B}\left(X^{k}\right)$ (for any $k \geq 0$ ) as the ring of invariants in $\mathrm{H}^{\bullet}\left(X^{k}, \mathbb{Q}\right) \cong \wedge^{\bullet}\left(\mathrm{H}^{\vee}, \oplus k\right)$ under the action of the special MumfordTate group (see Remark 2.1.12). If this Hodge ring is generated by divisor classes, the Hodge conjecture for $X^{k}$ is true.

To illustrate this method, let us carry it out in the simplest case, namely when $X$ is an elliptic curve with $\operatorname{End}^{0}(X)=\mathbb{Q}$. As before, let $\mathrm{H}=\mathrm{H}^{1}(X, \mathbb{Q})$. We know that $\mathrm{MT}(X)$ is a connected reductive subgroup of $\mathrm{GL}(\mathrm{H}) \cong \mathrm{GL}_{2, \mathbb{Q}}$ containing the homotheties. The only such subgroup $M$ for which $\operatorname{End}(\mathrm{H})^{M}=\mathbb{Q} \cdot \operatorname{id}_{\mathrm{H}}$ is $\mathrm{GL}(\mathrm{H})$ itself; hence $\operatorname{MT}(X)=\mathrm{GL}(\mathrm{H})$ and therefore $\operatorname{SMT}(X)=\mathrm{SL}(\mathrm{H})$. Now we determine 
the Hodge ring of $X^{k}$ as the ring of $\mathrm{SL}(\mathrm{H})$-invariants in $\wedge^{\bullet}\left(\mathrm{H}^{\vee} \oplus k\right)$. It is a classical result from invariant theory that if $V$ is the standard representation of $\mathrm{SL}_{2}$, the ring of $\mathrm{SL}_{2}$-invariants in $\wedge^{\bullet}\left(V^{\oplus k}\right)$ is generated by its elements in degree 2 . This means precisely that the Hodge ring $\mathscr{B}\left(X^{k}\right)$ is generated by divisor classes, and we conclude that the Hodge conjecture is true for all powers of $X$.

The example just given is only the tip of the iceberg. There is a rich literature on the topic, and wonderful results have been obtained based on such methods. See for instance [58], [59], [63], [64], [70], [88], [89], [90]. The method has its limitations, though. Already for abelian fourfolds, there are cases where the Hodge ring is not generated by divisor classes (see for instance [58], [107]), and apart from a couple of exceptions (see [72], [74], [99]) the Hodge conjecture is not known to be true in these cases. Also, in general it is not possible to determine $\operatorname{MT}(X)$ based only on information about $\operatorname{End}^{0}(X)$. We will further discuss what is known in Section 2.4.

\subsection{Galois representations}

We now turn to the arithmetic cousin of Hodge theory. In what follows, $K$ is a field, $K \subset K^{\mathrm{s}}$ is a separable closure, and we write $\Gamma_{K}=\operatorname{Gal}\left(K^{\mathrm{s}} / K\right)$. Further, $\ell$ denotes a prime number different from $\operatorname{char}(K)$.

Algebraic Galois groups. We write $\operatorname{Rep}\left(\Gamma_{K}\right)_{\mathbb{Q}_{\ell}}$ for the category of continuous representations of $\Gamma_{K}$ on finite dimensional $\mathbb{Q}_{\ell}$-vector spaces. An object in this category is given by a homomorphism

$$
\rho: \Gamma_{K} \rightarrow \mathrm{GL}(H)
$$

(with $H$ a $\mathbb{Q}_{\ell}$-vector space of finite dimension) which is continuous with respect to the $\ell$-adic topology on the target and the Krull topology on $\Gamma_{K}$. There is an obvious tensor product, and this makes $\operatorname{Rep}\left(\Gamma_{K}\right)_{\mathbb{Q}_{\ell}}$ a neutral Tannakian category. The forgetful functor $\operatorname{Rep}\left(\Gamma_{K}\right)_{\mathbb{Q}_{\ell}} \rightarrow \operatorname{Vec}_{\mathbb{Q}_{\ell}}$ is a fibre functor. With $\rho$ as above, the image $\operatorname{Im}(\rho)$ is an $\ell$-adic Lie subgroup of $\operatorname{GL}(H)$. We define

$$
G_{\ell}(H) \subset \mathrm{GL}(H)
$$

to be the Zariski closure of $\operatorname{Im}(\rho)$. By definition, this is a linear algebraic group over $\mathbb{Q}_{\ell}$, and it is the smallest algebraic subgroup $G \subset \mathrm{GL}(H)$ such that the representation $\rho$ factors through $G\left(\mathbb{Q}_{\ell}\right)$. This $G_{\ell}(H)$ is the algebraic group that in the Tannakian formalism corresponds to the Tannakian subcategory $\langle H\rangle \subset \operatorname{Rep}\left(\Gamma_{K}\right)_{\mathbb{Q}_{\ell}}$, with the forgetful functor as fibre functor; hence we have an equivalence of tensor categories between $\langle H\rangle$ and $\operatorname{Rep}\left(G_{\ell}(H)\right)$. In particular, we have the following analogue of property 2.1.3:

Key Property 2.2.1. For $r, s \in \mathbb{N}$, let $T^{r, s}=H^{\otimes r} \otimes\left(H^{\vee}\right)^{\otimes s}$. Let $T$ be any space of the form $T^{r_{1}, s_{1}} \oplus \cdots \oplus T^{r_{k}, s_{k}}$, and let $V \subset T$ be a $\mathbb{Q}_{\ell}$-subspace. Then $V$ is stable under the action of $G_{\ell}(H)$ on $T$ if and only if $V$ is stable under the action of $\Gamma_{K}$ (and is therefore a sub-object of $T$ in $\left.\operatorname{Rep}\left(\Gamma_{K}\right)_{\mathbb{Q}_{\ell}}\right)$.

As in Hodge theory, there are Tate twists. For this, let $\chi_{\ell}: \Gamma_{K} \rightarrow \mathbb{Z}_{\ell}^{\times}$be the $\ell$-adic cyclotomic character. For $H$ as above, its Tate twist $H(n)$ is given by the 
representation $\chi_{\ell}^{n} \cdot \rho$. Moreover, the Galois representations in which we are interested are pure and have a weight. We will not give details on this; see Deligne's seminal paper [36] or [48], Section 2.

Remarks 2.2.2. (i) Let $K \subset L$ be a finitely generated field extension and $L \subset L^{\mathrm{s}}$ a separable closure that contains $K^{\mathrm{s}}$. Then $K^{\prime}=L \cap K^{\mathrm{s}}$ is a finite extension of $K$ and we have $\Gamma_{L} \rightarrow \Gamma_{K^{\prime}} \subset \Gamma_{K}$. If we have a Galois representation $\rho$ as in (2.3), we may restrict it to $\Gamma_{L}$. The associated group $G_{\ell}(H)$ will in general become smaller, but the identity component $G_{\ell}^{0}(H)$ does not change.

(ii) If we denote by $\varpi_{0}=\varpi_{0}\left(G_{\ell}(H)\right)$ the (finite étale) group scheme of connected components of $G_{\ell}(H)$, the kernel of the composition

$$
\Gamma_{K} \rightarrow G_{\ell}(H)\left(\mathbb{Q}_{\ell}\right) \rightarrow \varpi_{0}\left(\mathbb{Q}_{\ell}\right)
$$

corresponds to a finite Galois extension $K \subset K^{\text {conn }}$. It has the property that, for $K \subset L$ as in (i), the algebraic group associated with $\left.\rho\right|_{\Gamma_{L}}$ is connected if and only if $K^{\text {conn }} \subset L$. In what follows we will sometimes assume that $K=K^{\text {conn }}$; this means that the group $G_{\ell}(H)$ is connected, and does not change if we replace $K$ by a finitely generated field extension. If the context requires it, we use the notation $K^{\operatorname{conn}}(\rho)$.

Galois representations on $\ell$-adic cohomology. We are primarily interested in Galois representations coming from $\ell$-adic cohomology of algebraic varieties. Keeping the above notation, let $X$ be a proper smooth scheme over $K$. The $\ell$-adic cohomology $\mathrm{H}^{m}\left(X_{K^{\mathrm{s}}}, \mathbb{Q}_{\ell}(n)\right)$ is defined as the cohomology in degree $m$ of a sheaf $\mathbb{Q}_{\ell}(n)$ on the pro-étale site $\left(X_{K^{\mathrm{s}}}\right)_{\text {pro-ét }}$ of $X_{K^{\mathrm{s}}}$. See [12]. (Before the pro-étale topology was introduced, one would work on the étale site of $X_{K^{\mathrm{s}}}$ and define $\mathrm{H}^{m}\left(X_{K^{\mathrm{s}}}, \mathbb{Z}_{\ell}(n)\right)$ as the limit over all $\mathrm{H}^{m}\left(X_{K^{\mathrm{s}} \text {,ét }},\left(\mathbb{Z} / \ell^{i} \mathbb{Z}\right)(n)\right)$; then set

$$
\mathrm{H}^{m}\left(X_{K^{\mathrm{s}}}, \mathbb{Q}_{\ell}(n)\right)=\mathrm{H}^{m}\left(X_{K^{\mathrm{s}}}, \mathbb{Z}_{\ell}(n)\right) \otimes \mathbb{Q}_{\ell} .
$$

This gives the same cohomology groups.)

Fixing $m$ and $n$, let us abbreviate $\mathrm{H}=\mathrm{H}^{m}\left(X_{K^{\mathrm{s}}}, \mathbb{Q}_{\ell}(n)\right)$. The Galois group $\Gamma_{K}=\operatorname{Gal}\left(K^{\mathrm{s}} / K\right)$ acts on $X_{K^{\mathrm{s}}}$, and by functoriality we obtain an action on the $\ell$-adic cohomology, i.e., a representation

$$
\rho_{\ell}=\rho_{\ell, \mathrm{H}^{m}}(X)(n): \Gamma_{K} \rightarrow \mathrm{GL}(\mathrm{H}) .
$$

This representation is continuous with respect to the $\ell$-adic topology on the target and the Krull topology on $\Gamma_{K}$, making it an object of $\operatorname{Rep}\left(\Gamma_{K}\right)_{\mathbb{Q}_{\ell}}$. If $X / K$ is geometrically irreducible of dimension $d$ then $\mathrm{H}^{\bullet}\left(X_{K^{\mathrm{s}}}, \mathbb{Q}_{\ell}\right)=\oplus_{n=0}^{2 d} \mathrm{H}^{n}\left(X_{K^{\mathrm{s}}}, \mathbb{Q}_{\ell}\right)$ is a graded-commutative algebra in $\operatorname{Rep}\left(\Gamma_{K}\right)_{\mathbb{Q}_{\ell}}$. The Galois representations of the form $\rho_{\ell, \mathrm{H}^{m}}(X)(n)$ constitute only a small part of the category $\operatorname{Rep}\left(\Gamma_{K}\right)_{\mathbb{Q}_{\ell}} ;$ we refer to [97] for an in-depth discussion of the properties they enjoy.

Let $X$ be a complete nonsingular variety over a separably closed field $\Omega$. Fix integers $m$ and $n$, and write $\mathrm{H}=\mathrm{H}^{m}\left(X, \mathbb{Q}_{\ell}(n)\right)$. We associate with $\mathrm{H}$ a connected algebraic group $G_{\ell}^{0}(\mathrm{H}) \subset \mathrm{GL}(\mathrm{H})$, as follows. Choose any subfield $K \subset \Omega$ that is of finite type over the prime field, and a model $X_{K}$ of $X$ over $K$. (This means: a $K$ scheme $X_{K}$ together with an isomorphism $X_{K} \otimes_{K} \Omega \cong X$.) Let $K^{\text {s }}$ be the separable 
closure of $K$ in $\Omega$. Writing $X_{K^{\mathrm{s}}}=X_{K} \otimes_{K} K^{\mathrm{s}}$, we have a canonical isomorphism $\mathrm{H}^{m}\left(X_{K^{\mathrm{s}}}, \mathbb{Q}_{\ell}(n)\right) \stackrel{\sim}{\longrightarrow} \mathrm{H}=\mathrm{H}^{m}\left(X, \mathbb{Q}_{\ell}(n)\right)$. Taking this as an identification, we get a representation $\rho_{\ell}$ as in (2.4), and, as before, we define $G_{\ell}^{0}(\mathrm{H}) \subset \mathrm{GL}(\mathrm{H})$ to be the identity component of the Zariski closure of its image. By the Remark 2.2.2(i), the group thus obtained is independent of how we choose the model $X_{K} / K$.

Definition 2.2.3. With notation as above, an element $\xi \in \mathrm{H}$ is called a Tate class if $x$ is invariant under $G_{\ell}^{0}(\mathrm{H})$.

Remarks 2.2.4. (i) Taking a model $X_{K} / K$ as above, $\xi$ is a Tate class if and only if $\xi$ is invariant under the action of $\Gamma_{K^{\prime}}$ for some finite extension $K \subset K^{\prime}$.

(ii) The Galois representation on $\mathrm{H}^{m}\left(X_{K^{\mathrm{s}}}, \mathbb{Q}_{\ell}(n)\right)$ is pure of weight $m-2 n$. This implies that there can be non-zero Tate classes in $\mathrm{H}$ only if $m=2 n$.

Cycle classes. Still with $X$ a complete nonsingular variety over $\Omega$, to every algebraic cycle $Z$ of codimension $n$ we can associate a cohomology $\operatorname{class} \operatorname{cl}(Z) \in$ $\mathrm{H}^{2 n}\left(X, \mathbb{Q}_{\ell}(n)\right)$. The cohomology class that we obtain is a Tate class that only depends on $Z$ modulo rational equivalence; so we obtain a map

$$
c l_{\ell}: \mathrm{CH}^{n}(X) \otimes \mathbb{Q}_{\ell} \rightarrow\left\{\text { Tate classes in } \mathrm{H}^{2 n}\left(X, \mathbb{Q}_{\ell}(n)\right)\right\} .
$$

The Tate conjecture expresses that all Tate classes in the cohomology should arise from algebraic cycles in this way.

Conjecture 2.2.5 (The Tate Conjecture). Let $X$ be a complete nonsingular algebraic variety over a separably closed field $\Omega$. Then for every $n \geq 0$ the group $G_{\ell}^{0}(\mathrm{H})$ associated with $\mathrm{H}=\mathrm{H}^{2 n}\left(X, \mathbb{Q}_{\ell}(n)\right)$ is reductive and the cycle class map (2.5) is surjective.

If a specific value for $n$ is chosen, we refer to this conjecture as the Tate conjecture for cycles in codimension $n$ on $X$.

Remarks 2.2.6. (i) If $\tilde{\Omega}$ is a separably closed field containing $\Omega$, the Tate conjecture for $X$ is equivalent to the Tate conjecture for $X_{\tilde{\Omega}}$.

(ii) We have formulated the conjecture for (complete, nonsingular) varieties over a separably closed field. This should not obscure the fact that the Tate conjecture is really a statement about varieties over fields that are finitely generated over their prime field. The TC in this form is equivalent to the assertion that for every smooth projective $X$ over a finitely generated field $K$, every class $\xi \in \mathrm{H}^{2 n}\left(X_{K^{\mathrm{s}}}, \mathbb{Q}_{\ell}(n)\right)$ that is invariant under the action of $\Gamma_{K}$ is in the image of the $\ell$-adic cycle class map $\mathrm{CH}^{n}(X) \otimes \mathbb{Q}_{\ell} \rightarrow \mathrm{H}^{2 n}\left(X, \mathbb{Q}_{\ell}(n)\right)$.

(iii) The group $G_{\ell}^{0}(H)$ is reductive if and only if the representation $\rho_{\ell}$ is completely reducible (=semisimple).

(iv) It is proven in [57] that if for all complete nonsingular varieties over $\overline{\mathbb{Q}}$ and all $n \geq 0$ the cycle class map (2.5) is surjective, then for every complete nonsingular $X$ over a finitely generated field $K$ of characteristic 0 the Galois representation $\rho_{\ell}$ of (2.4) is completely reducible (for all $m$ and $n$ ). 
For $n=0$ and $n=d=\operatorname{dim}(X)$ the Tate conjecture is "trivially" true: choosing a model $X / K$ as before, $\mathrm{H}^{0}\left(X_{K^{\mathrm{s}}}, \mathbb{Q}\right)$ and $\mathrm{H}^{2 d}\left(X_{K^{\mathrm{s}}}, \mathbb{Q}(d)\right)$ are both isomorphic to $\mathbb{Q}_{\ell}(0)$ as Galois representations (assuming $X$ to be irreducible), and are spanned by the class of $X$, and the class of a point on $X$, respectively. Already for $n=1$ the Tate conjecture is not known in general. We refer to [96] for a much more detailed discussion of the Tate conjecture.

For abelian varieties, we have the following deep result, due to Tate [95] (over finite fields), Zarhin [110], [111] (over finitely generated fields of characteristic $p>2$ ), Mori (the case $p=2$ ), and Faltings [38] (finitely generated fields of characteristic 0). In the statement of the theorem we consider, for $X / K$ an abelian variety, the $\ell$ adic Tate module $T_{\ell}(X)$, which is the first integral $\ell$-adic homology group. We have $\mathrm{H}^{1}\left(X_{K^{\mathrm{s}}}, \mathbb{Q}_{\ell}\right) \cong \operatorname{Hom}\left(T_{\ell}(X), \mathbb{Q}_{\ell}\right)$ as Galois-modules, and since $\mathrm{H}^{\bullet}\left(X_{K^{\mathrm{s}}}, \mathbb{Q}_{\ell}\right)$ is the exterior algebra on $\mathrm{H}^{1}\left(X_{K^{\mathrm{s}}}, \mathbb{Q}_{\ell}\right)$ the entire cohomology ring of $X$ is determined by its Tate module.

Theorem 2.2.7. Let $X$ and $Y$ be abelian varieties over a finitely generated field $K$, and let $\ell$ be a prime number different from $\operatorname{char}(K)$. Then $T_{\ell}(X) \otimes \mathbb{Q}_{\ell}$ and $T_{\ell}(Y) \otimes \mathbb{Q}_{\ell}$ are semisimple as representations of $\Gamma_{K}=\operatorname{Gal}\left(K^{\mathrm{s}} / K\right)$ and the natural homomorphism

$$
\operatorname{Hom}(X, Y) \otimes \mathbb{Z}_{\ell} \longrightarrow \operatorname{Hom}_{\Gamma_{K}}\left(T_{\ell}(X), T_{\ell}(Y)\right)
$$

is an isomorphism.

Corollary 2.2.8. Let $X$ be an abelian variety over a separately closed field $\Omega$. Let $\ell$ be a prime number different from $\operatorname{char}(\Omega)$. Then the $\ell$-adic Tate conjecture for divisor classes on $X$ is true.

To deduce this from Theorem 2.2.7 is a little exercise in the theory of abelian varieties. Consider an abelian variety $X$ over a finitely generated field $K$. If $X^{t}$ is the dual abelian variety, we have a perfect Galois-equivariant pairing $E: T_{\ell}(X) \times$ $T_{\ell}\left(X^{t}\right) \rightarrow \mathbb{Z}_{\ell}(1)$, which gives an identification $T_{\ell}\left(X^{t}\right) \stackrel{\sim}{\sim} T_{\ell}(X)^{\vee}(1)$ by $y \mapsto E(-, y)$. By doing the same with $X^{t}$ instead of $X$ we get $T_{\ell}(X) \stackrel{\sim}{\longrightarrow} T_{\ell}\left(X^{t}\right)^{\vee}(1)$ (which is the negative of the identification given by $x \mapsto E(x,-))$. With these identifications one checks that $\mathrm{H}^{2}\left(X_{K^{\mathrm{s}}}, \mathbb{Z}_{\ell}(1)\right)$ is naturally isomorphic to the space $\operatorname{Hom}^{\mathrm{sym}}\left(T_{\ell}(X), T_{\ell}\left(X^{t}\right)\right)$ of homomorphisms $f: T_{\ell}(X) \rightarrow T_{\ell}\left(X^{t}\right)$ with $f^{\vee}(1)=f$. The isomorphism of Theorem 2.2.7 restricts to an isomorphism

$$
\operatorname{Hom}^{\mathrm{sym}}\left(X, X^{t}\right) \otimes \mathbb{Z}_{\ell} \stackrel{\sim}{\longrightarrow} \operatorname{Hom}_{\Gamma_{K}}^{\mathrm{sym}}\left(T_{\ell}(X), T_{\ell}\left(X^{t}\right)\right)
$$

where on the left we consider the homomorphisms $F$ with $F^{t}=F$. On the other hand, it is known that the map $\operatorname{NS}(X) \rightarrow \operatorname{Hom}^{\mathrm{sym}}\left(X, X^{t}\right)$ that sends the class of a line bundle $L$ to the "Mumford homomorphism" $\varphi_{L}: X \rightarrow X^{t}$ given by $x \mapsto$ $\left[t_{x}^{*}(L) \otimes L^{-1}\right]$ is an isomorphism. As the composition

$$
\mathrm{NS}(X) \rightarrow \operatorname{Hom}^{\mathrm{sym}}\left(X, X^{t}\right) \rightarrow \operatorname{Hom}_{\Gamma_{K}}^{\mathrm{sym}}\left(T_{\ell}(X), T_{\ell}\left(X^{t}\right)\right) \rightarrow \mathrm{H}^{2}\left(X_{K^{\mathrm{s}}}, \mathbb{Q}_{\ell}(1)\right)^{\Gamma_{K}}
$$

is the map that associates to $L$ its first Chern class, this gives the corollary. 
Remark 2.2.9. Over fields of characteristic $p>0$ there is also a $p$-adic version of the Tate conjecture. Over finite fields this is due to Tate. See de Jong's paper [29], Section 2 for the general case.

Remark 2.2.10. As already remarked, the Galois representations that are of interest have many good properties. One aspect of this is that, for $X / K$ smooth proper, the collection of Galois representations $\rho_{\ell, \mathrm{H}^{m}}(X)(n)$ forms a strictly compatible system of $\ell$-adic representations in the sense defined by Serre in [77], Chapter I. This has given rise to numerous important results, especially about the independence of $\ell$ of properties of the associated $\ell$-adic algebraic Galois groups $G_{\ell}$. For the key results, most of which are due to Serre or to Larsen and Pink, we refer to [26], [81], [82], [51], [52]. Let us already note that for motives in the sense of André (or for motives for absolute Hodge classes) it is not known in general if the associated $\ell$-adic Galois representations form a strictly compatible system; see Remark 3.2.1.

\subsection{The Mumford-Tate conjecture for complex varieties}

Let $X$ be a complete nonsingular complex algebraic variety. Fixing integers $m$ and $n$, let $\mathrm{H}=\mathrm{H}^{m}(X, \mathbb{Q}(n))$, which is a polarizable pure $\mathbb{Q}$-Hodge structure. Let $\mathrm{MT}(\mathrm{H}) \subset$ $\mathrm{GL}(\mathrm{H})$ denote the Mumford-Tate group. On the other hand, for $\ell$ a prime number, let $\mathrm{H}_{\ell}=\mathrm{H}^{m}\left(X, \mathbb{Q}_{\ell}(n)\right)$ be the $\ell$-adic cohomology, to which we have associated a group $G_{\ell}^{0}\left(\mathrm{H}_{\ell}\right) \subset \mathrm{GL}\left(\mathrm{H}_{\ell}\right)$. There is a comparison theorem between singular and étale cohomology that gives us a canonical isomorphism of $\mathbb{Q}_{\ell}$-vector spaces $i: \mathrm{H} \otimes \mathbb{Q}_{\ell} \sim$ $\mathrm{H}_{\ell}$. Let $\gamma: \mathrm{GL}(\mathrm{H}) \otimes \mathbb{Q}_{\ell} \stackrel{\mathrm{GL}}{\sim}\left(\mathrm{H}_{\ell}\right)$ denote the induced isomorphism of algebraic groups over $\mathbb{Q}_{\ell}$.

Conjecture 2.3.1 (The Mumford-Tate Conjecture). The isomorphism $\gamma$ restricts to an isomorphism

$$
\mathrm{MT}(\mathrm{H}) \otimes \mathbb{Q}_{\ell} \stackrel{?}{\sim} G_{\ell}^{0}\left(\mathrm{H}_{\ell}\right)
$$

We refer to the conjectural equality of $\mathrm{MT}(\mathrm{H}) \otimes \mathbb{Q}_{\ell}$ and $G_{\ell}^{0}\left(\mathrm{H}_{\ell}\right)$ as the MumfordTate conjecture for $\mathrm{H}^{m}(X)(n)$. See Conjecture 3.2.4 below for a more general version.

In what follows we use the abbreviations HC, TC and MTC for the Hodge, Tate and Mumford-Tate conjectures.

Proposition 2.3.2. Let $X$ be a complete nonsingular complex algebraic variety, and let $n \geq 0$ be an integer. Assume the Mumford-Tate conjecture for $\mathrm{H}^{2 n}(X)(n)$ is true. Then

$H C$ in codimension $n$ for $X \Longleftrightarrow T C$ in codimension $n$ for $X$.

Proof. Keep the notation introduced above, with $m=2 n$. If the MTC is true for $\mathrm{H}^{2 n}(X)(n)$ then the isomorphism $i: \mathrm{H} \otimes \mathbb{Q}_{\ell} \stackrel{\sim}{\longrightarrow} \mathrm{H}_{\ell}$ restricts to an isomorphism

$\{$ Hodge classes in $\mathrm{H}\} \otimes \mathbb{Q}_{\ell} \sim\left\{\right.$ Tate classes in $\left.\mathrm{H}_{\ell}\right\}$.

The proposition follows because, via $i$, the $\ell$-adic cycle class map (2.5) is identified with the $\mathbb{Q}_{\ell}$-linear extension of the cycle class map (2.2). 
As an example of how this can be useful, recall that the Hodge conjecture is known in codimension 1 (Lefschetz's theorem on divisor classes), whereas the Tate conjecture is not, in general, known for divisor classes. Hence the MTC for $\mathrm{H}^{2}(X)(1)$ implies the TC in codimension 1 for $X$.

Remark 2.3.3. There is a partial converse to the proposition. We keep the above notation, with $m=2 n$. If the $\mathrm{HC}$ and TC on $X$ are true in codimension $n$, it follows that $\mathrm{H}^{\mathrm{MT}(\mathrm{H})} \otimes \mathbb{Q}_{\ell} \sim\left(\mathrm{H}_{\ell}\right)^{G_{\ell}^{0}\left(\mathrm{H}_{\ell}\right)}$. In general this does not suffice to conclude that the MTC is true for $\mathrm{H}^{2 n}(X)(n)$. The reason is that the groups $\mathrm{MT}(\mathrm{H})$ and $G_{\ell}^{0}\left(\mathrm{H}_{\ell}\right)$ control all tensor spaces built from $\mathrm{H}$ and $\mathrm{H}_{\ell}$ respectively. These tensor spaces occur in the cohomology of powers of $X$. One can show that if for all $k \geq 1$ the HC and TC are true for $X^{k}$ in codimension $k n$ then the MTC is true for $\mathrm{H}^{2 n}(X)(n)$.

\subsection{Some known results (1)}

This is the first of three sections (see 3.3 and 4.4 for the other) in which we briefly discuss what is known about the Mumford-Tate conjecture and give some pointers to the literature. These sections have no pretense at completeness, and we apologize in advance to authors whose contributions we fail to give the attention they deserve.

Representation-theoretic constraints. The method that was outlined in Example 2.1.13 lies at the basis of many interesting results about abelian varieties. Let $(X, \lambda)$ be a $g$-dimensional polarized complex abelian variety, and write $\mathrm{H}=\mathrm{H}_{1}(X, \mathbb{Q})$. One should like to determine $\operatorname{MT}(X)$ based on information about the endomorphism algebra $\operatorname{End}^{0}(X)$. The polarization $\lambda$ (in the sense of the theory of abelian varieties) gives rise to a polarization $\phi: H \times H \rightarrow \mathbb{Q}(1)$ (in the sense of Hodge theory), which, viewed as an element of $\left(\mathrm{H}^{\vee}\right)^{\otimes 2}(1)$, is a Hodge class. This gives that $\operatorname{MT}(X)$ is a connected reductive subgroup of the group of symplectic similitudes $\operatorname{CSp}(\mathrm{H}, \phi)$, and as discussed, $\operatorname{End}^{0}(X)$ is the algebra of $\mathrm{MT}(X)$-invariants in $\operatorname{End}(\mathrm{H})$, i.e., the algebra of endomorphisms of $\mathrm{H}$ that commute with $\operatorname{MT}(X)$. This can be refined in the following way.

In general, if $H$ is a polarizable $\mathbb{Q}$-Hodge structure, write $M=\operatorname{MT}(X)$ and choose a maximal torus $T \subset M$. We have a root datum with underlying lattice $X^{*}(T)$, and the tautological representation $M_{\mathbb{C}} \rightarrow \mathrm{GL}(H \otimes \mathbb{C})$ is given by a multiset $\mathcal{P}$ of weights in $X^{*}(T) \otimes \mathbb{R}$. As in Remark 2.1.5, we have a cocharacter $\mu: \mathbb{G}_{\mathrm{m}} \rightarrow M_{\mathbb{C}}$. Some conjugate $\nu$ of it factors through $T_{\mathbb{C}}$ and hence gives rise to a linear map $\nu^{*}: X^{*}(T) \otimes \mathbb{R} \rightarrow \mathbb{R}$. We then know that the image of $\mathcal{P}$ under $\nu^{*}$ is precisely the set of integers $-p$ counted with multiplicity the Hodge number $h^{p, q}$. Moreover, $\nu$ is defined over $\overline{\mathbb{Q}}$ and $M_{\overline{\mathbb{Q}}}$ is generated by $\nu$ together with its $\operatorname{Gal}(\overline{\mathbb{Q}} / \mathbb{Q})$-conjugates. In this way, knowing the Hodge numbers gives restrictions on the representation.

For a $g$-dimensional abelian variety this means that we have a number of projections $X^{*}(T) \otimes \mathbb{R} \rightarrow \mathbb{R}$, corresponding to $\nu$ and its Galois-conjugates, under each of which $\mathcal{P}$ has as image the multiset $\left\{0^{g}, 1^{g}\right\}$. This leads to the following result.

Theorem 2.4.1. Let $X$ be a complex abelian variety, $\mathrm{H}=\mathrm{H}_{1}(X, \mathbb{Q})$ and $M=\operatorname{MT}(X)$. Write $M_{\mathbb{C}}=Z \cdot M_{1} \cdot M_{2} \cdots M_{r}$, where $Z$ is the center and $M_{1}, \ldots, M_{r}$ are the simple factors of $M_{\mathbb{C}}$. Let $W$ be an irreducible representation of $M_{\mathbb{C}}$ that occurs in $\mathrm{H}_{\mathbb{C}}$. 
(i) All simple factors $M_{i}$ are of Lie type $A, B, C$ or $D$.

(ii) Write $W=\chi \otimes W_{1} \otimes \cdots \otimes W_{r}$, where $\chi$ is a character of $Z$ and $W_{i}$ is an irreducible representation of $M_{i}$. If the representation $W_{i}$ is non-trivial, its highest weight (after choosing a basis for the root system) is a minuscule weight.

For the notion of a minuscule weight, see Bourbaki [16], Chap. VIII, $\S 7$, no. 3. The theorem can be found in [33], Section 1.2 or, with more details, in [80], Section 3.

For Hodge structures of arbitrary level, a detailed analysis of the representationtheoretic constraints that are obtained from the Hodge numbers can be found in the work of Zarhin [112].

When we try to obtain analogous results about the groups $G_{\ell}$ associated with the Galois representation on $\mathrm{H}_{1}\left(X, \mathbb{Q}_{\ell}\right)=T_{\ell}(X) \otimes \mathbb{Q}_{\ell}$, it is not clear a priori how to proceed. While the Mumford-Tate group can be described as the smallest algebraic group receiving the cocharacter $\mu$, this has no direct analogue in the $\ell$-adic setting. However, Pink [67] has proven that such representation-theoretic constraints also hold in the $\ell$-adic setting, by making very clever use of cocharacters associated to local Galois representations via Hodge-Tate theory. Specifically, [67], Theorem 3.18, is precisely what is needed to obtain analogues of the results of Deligne, Serre and Zarhin in the $\ell$-adic setting. For abelian varieties, this is discussed in detail in [67], Sections 4 and 5 . In particular, for abelian varieties $X$ with $\operatorname{End}(X)=\mathbb{Z}$ satisfying some numerical conditions on $\operatorname{dim}(X)$, Pink proves that the HC, TC and MTC are true for all powers of $X$; this improves earlier results of Serre [82], [83] and Tankeev [94]. For some other types of endomorphism algebras, analogous results have been obtained for instance in [9] and [10]. See [55], Section 2, for an application of Pink's results in the context of motives of K3 type.

Some results. Let us now briefly mention some other results about the HC, TC and MTC for abelian varieties. Throughout, $X$ denotes a complex abelian variety and $\mathrm{H}$ (resp. $\mathrm{H}_{\ell}$ ) denotes the first homology group (singular, resp. $\ell$-adic) of $X$. In some cases we only state a precise result in the Hodge-theoretic setting, leaving it to the reader to formulate the $\ell$-adic analogue.

2.4.2. Taking the isomorphism $\gamma: \mathrm{GL}(\mathrm{H}) \otimes \mathbb{Q}_{\ell} \stackrel{\mathrm{GL}}{\sim}\left(\mathrm{H}_{\ell}\right)$ of Section 2.3 as an identification, we have

$$
G_{\ell}^{0}(X) \subset \operatorname{MT}(X) \otimes \mathbb{Q}_{\ell}
$$

This is an immediate consequence of a much deeper result, Theorem 3.3.1; we will return to this in Section 3.3.

2.4.3. With SMT the special Mumford-Tate group, as in Remark 2.1.12, we have $\operatorname{MT}(X)=\mathbb{G}_{\mathrm{m}} \cdot \operatorname{SMT}(X)$ and $\operatorname{SMT}(X) \subset \operatorname{Sp}(\mathrm{H}, \phi)$. It follows from relation $(2.1)$ that the center $Z$ of $\operatorname{SMT}(X)$ is contained in $\operatorname{End}^{0}(X) \cap \operatorname{Sp}(\mathrm{H}, \phi)$. Using this one finds that $\operatorname{SMT}(X)$ is semisimple if $\operatorname{End}^{0}(X)$ has no factors of Type IV. Similarly, the identity component of $G_{\ell}(X) \cap \mathrm{SL}\left(\mathrm{H}_{\ell}\right)$ is semisimple if there are no factors of Type IV. 
2.4.4. The abelian variety $X$ is of CM type if and only if $\operatorname{MT}(X)$ is a torus, if and only if $G_{\ell}^{0}(X)$ is a torus. The MTC is true for abelian varieties of CM type. This result is proven by Pohlmann in [68]; as is nicely explained in [109], it is in fact a consequence of the results about complex multiplication due to Shimura-Taniyama and Weil. In 3.3.2 we will discuss a much more general result.

2.4.5. It was proven by Larsen and Pink that the Mumford-Tate conjecture for abelian varieties is independent of $\ell$ : if it is true for one $\ell$, it is true for all. See [52], Theorem 4.3. We will return to this in Section 3.3.

2.4.6. Let $\tilde{L}(X)$ denote the algebraic subgroup of $\operatorname{Sp}(H, \phi)$ consisting of elements that commute with the action of $\operatorname{End}^{0}(X)$. Let $L(X)$ be the identity component of $\tilde{L}(X)$. On the other hand, we can define an invariant $\operatorname{rdim}(X)$, called the reduced dimension of $X$. If $X$ is simple and $k$ is the Schur index of the central simple algebra $\operatorname{End}^{0}(X)$, the reduced dimension is given by

$$
\operatorname{rdim}(X)= \begin{cases}\operatorname{dim}(X) & \text { if } \operatorname{End}^{0}(X) \text { is of Albert Type I or III; } \\ \operatorname{dim}(X) / k & \text { if } \operatorname{End}^{0}(X) \text { is of Albert Type II or IV }\end{cases}
$$

For a general $X$, let $X \sim X_{1}^{n_{1}} \times \cdots \times X_{r}^{n_{r}}$ be the decomposition (up to isogeny) of $X$ into simple factors with $X_{i} \not X_{j}$ for $i \neq j$; then one defines $\operatorname{rdim}(X)=\operatorname{rdim}\left(X_{1}\right)+$ $\cdots+\operatorname{rdim}\left(X_{r}\right)$. Combining results of Murty [64] and Hazama [41], we find that the following properties are equivalent: (a) the Hodge ring of $X^{n}$ is generated by divisor classes for all $n \geq 1$, (b) $X$ has no simple factors of Type III and $\operatorname{MT}(X)=L(X)$, and (c) the special Mumford-Tate group $\operatorname{SMT}(X)$ has rank equal to $\operatorname{rdim}(X)$. The $\ell$-adic analogue of this is also true. If (a)-(c) are true for $X_{1}$ and $X_{2}$, they are also true for the product $X_{1} \times X_{2}$.

2.4.7. Let $X$ be a simple abelian variety of dimension 1 or of prime dimension $p$. Then the HC, TC and MTC are true for all powers of $X$. For elliptic curves this is due to Serre; the essential ingredient is [76], Théorème 1. (Once one has this result, one can argue as in Example 2.1.13.) The result about simple abelian varieties of prime dimension is due to Tankeev; see [70], [90], [91]. Note that in this case there are only four possibilities for the endomorphism algebra $\operatorname{End}^{0}(X)$ : it is $\mathbb{Q}$, a totally real field of degree $p$, an imaginary quadratic field, or a CM field of degree $2 p$. Especially in the first two cases we see Theorem 2.4.1 coming into action. For instance, if $\operatorname{End}^{0}(X)=\mathbb{Q}$, the first homology group $\mathrm{H}$ is an absolutely simple symplectic representation of the special Mumford-Tate group, of dimension $2 p$. We find that $\operatorname{SMT}(X)$ is absolutely simple of Lie type A, B, C or D, and that $\mathrm{H}$ is defined by a minuscule highest weight. Inspecting the table of minuscule weights and their dimensions (see [80], Annexe, for instance), one then concludes that $\operatorname{SMT}(X)$ is necessarily the full symplectic group $\operatorname{Sp}(\mathrm{H}, \phi)$. In the $\ell$-adic setting, the same arguments apply. One concludes by using the results of Hazama and Murty mentioned in 2.4.6.

For simple abelian varieties, the case of dimension 4 is the next one to consider. (We will discuss the non-simple case in Section 3.3.) For $X$ simple of dimension 4 the MTC is known, except when $\operatorname{End}(X)=\mathbb{Z}$. In that case, a construction of 
Mumford (see [61], § 4) implies that knowing the endomorphism algebra does not suffice to determine the Mumford-Tate group $\mathrm{MT}(X)$ or the $\ell$-adic algebraic Galois groups $G_{\ell}^{0}(X)$. This leaves open the possibility that $G_{\ell}^{0}(X)$ is strictly contained in $\operatorname{MT}(X) \otimes \mathbb{Q}_{\ell}$, and it is not known how to exclude this. Still with $X$ simple of dimension 4, the $\mathrm{HC}$ and $\mathrm{TC}$ are known for $X$ for some types of endomorphism algebras, but not in general. See [58].

2.4.8. A complex abelian variety $X$ gives rise to a Shimura datum $(G, \mathscr{X})$ with $G=\operatorname{MT}(X)$. The associated adjoint Shimura datum is a product of simple adjoint Shimura data $\left(H_{j}, \mathscr{Y}_{j}\right)$. Vasiu [102] has proven a very general result that if certain Lie types do not occur among the $\left(H_{j}, \mathscr{Y}_{j}\right)$, the Mumford-Tate conjecture for $X$ is true.

\section{Motives and the Motivic Mumford-Tate conjecture}

\subsection{Motives and motivic Galois groups}

It is time to bring motives into the discussion. This will give us a better understanding of why we expect the MTC to be true, and will also lead to a stronger variant of the MTC.

Motivated cycles. In the past two decades the theory of motives has seen spectacular developments. As an excellent starting point for further reading we recommend the book [6]. In this article we shall only consider pure motives as defined by André in [5]. His definition is modelled after the classical construction of Grothendieck. In Grothendieck's approach one is confronted with the problem that we do not know how to construct enough algebraic cycles. André's key insight is that one obtains a theory with almost all expected properties by formally adjoining the Lefschetz operator $*_{L}$ for every smooth projective variety $X$ equipped with an ample class $L$.

We briefly review André's construction, referring to the original paper [5] for many more details and for generalizations. Let $K$ be a field, and denote by $\operatorname{SmPr}(K)$ the category of smooth projective $K$-schemes. We fix a Weil cohomology theory $\mathrm{H}$ on smooth projective $K$-schemes, with coefficient field Q of characteristic 0 . We further assume that the hard Lefschetz theorem holds for this theory; by this we mean that for every irreducible $X$ in $\operatorname{SmPr}(K)$ of dimension $d$ and every ample class $L \in \mathrm{H}^{2}(X)$ the maps $L^{i}: \mathrm{H}^{d-i}(X) \rightarrow \mathrm{H}^{d+i}(X)$ are isomorphisms. This holds, for instance, if for $\mathrm{H}$ we take $\ell$-adic cohomology for some prime number $\ell \neq \operatorname{char}(K)$.

A motivated cycle on $X$ is defined to be a class $\xi \in \mathrm{H}(X)$ that can be obtained via the following procedure:

- Let $Y$ be another variety in $\operatorname{SmPr}(K)$ and choose ample classes $L_{X}$ on $X$ and $L_{Y}$ on $Y$.

- On $X \times Y$, consider the ample class $L=\operatorname{pr}_{X}^{*}\left(L_{X}\right)+\operatorname{pr}_{Y}^{*}\left(L_{Y}\right)$ and let $*_{L}$ denote the associated Lefschetz involution on $\mathrm{H}(X \times Y)$. By definition, if we write a 
cohomology class $x \in \mathrm{H}^{j}(X \times Y)$ as $x=\sum L^{k}\left(x_{j-2 k}\right)$ with $x_{j-2 k} \in \mathrm{H}_{\operatorname{prim}}^{j-2 k}(X \times$ $Y)$, then

$$
*_{L}(x)=\sum L^{\operatorname{dim}(X \times Y)-j+k}\left(x_{j-2 k}\right),
$$

which may formally be written as $L^{\operatorname{dim}(X \times Y)-j}(x)$.

- Let $\alpha$ and $\beta$ be algebraic cycle classes on $X \times Y$ with $\mathbb{Q}$-coefficients, and take

$$
\xi=\operatorname{pr}_{X, *}\left(\alpha \cup *_{L}(\beta)\right) \text {. }
$$

The set $A_{\text {mot }}(X)$ of motivated cycles is a graded $\mathbb{Q}$-subalgebra of $\mathrm{H}^{\text {even }}(X)$ that contains all classes of algebraic cycles.

Remarks 3.1.1. (i) The algebra of motivated cycles is constructed as a $\mathbb{Q}$-subalgebra of $\mathrm{H}(X)$ for some "reference cohomology theory" H. However, the algebras $A_{\operatorname{mot}}(X)$ that are obtained do not depend on the chosen cohomology theory; see [5], Section 2.3. (Note that the coefficient field $Q$ of $H$ may be bigger than $\mathbb{Q}$.)

(ii) In the above definition we have ignored Tate twists. If we include them we find that $A_{\text {mot }}^{r}(X)$ is a subspace of $\mathrm{H}^{2 r}(X)(r)$.

In the rest of the discussion, we assume char $(K)=0$.

André's category of motives. With algebraic cycle classes replaced by motivated cycles, the construction of the category $\operatorname{Mot}(K)$ is the classical one. For $X=\coprod_{\nu} X_{\nu}$ and $Y$ in $\operatorname{Sm} \operatorname{Pr}(K)$ with $X_{\nu}$ irreducible of dimension $d_{\nu}$, one defines the graded vector space $\operatorname{Corr}_{\text {mot }}^{\bullet}(X, Y)$ of motivated correspondences by the rule that $\operatorname{Corr}_{\text {mot }}^{r}(X, Y)=$ $\oplus_{\nu} A_{\text {mot }}^{d_{\nu}+r}\left(X_{\nu} \times Y\right)$. One shows that the usual rule for the composition of correspondences gives a graded map $\operatorname{Corr}_{\text {mot }}^{\bullet}(X, Y) \otimes \operatorname{Corr}_{\text {mot }}^{\bullet}(Y, Z) \rightarrow \operatorname{Corr}_{\text {mot }}^{\bullet}(X, Z)$.

A motive over $K$ is defined to be a triple $(X, e, n)$ with $X$ a smooth projective $K$ scheme, $e$ an idempotent in $\operatorname{Corr}_{\text {mot }}^{0}(X, X)$ and $n$ an integer. One thinks of $(X, e, n)$ as the motive that is cut out from $X$ by the projector $e$, and then Tate twisted by $n$. A morphism from $(X, e, n)$ to $\left(Y, e^{\prime}, n^{\prime}\right)$ is defined to be an element of the subspace

$$
e^{\prime} \circ \operatorname{Corr}_{\text {mot }}^{n^{\prime}-n}(X, Y) \circ e \subset \operatorname{Corr}_{\text {mot }}^{n^{\prime}-n}(X, Y) .
$$

In this way we obtain the category $\operatorname{Mot}(K)$ of motives (in the sense of André) over $K$.

In what follows we usually denote motives by a single bold letter, and if $\mathbf{M}=$ $(X, e, n)$ then we let $\mathbf{M}(m)=(X, e, n+m)$. If $\mathbf{M}=(X, e, n)$ and $\mathbf{M}^{\prime}=\left(Y, e^{\prime}, n^{\prime}\right)$ are motives, we define their tensor product to be $\mathbf{M} \otimes \mathbf{M}^{\prime}=\left(X \times_{K} Y, e \times e^{\prime}, n+n^{\prime}\right)$. We call $\mathbf{1}=(\operatorname{Spec}(K),[\Delta], 0)$ the unit motive.

We have a contravariant functor $\mathbf{H}: \operatorname{SmPr}(K)^{\text {op }} \rightarrow \operatorname{Mot}(K)$, sending $X$ to the motive $\mathbf{H}(X)=\left(X,\left[\Delta_{X}\right], 0\right)$ and sending a morphism $f: X \rightarrow Y$ to the class of the transpose graph $\left[{ }^{t} \Gamma_{f}\right] \in \operatorname{Corr}_{\text {mot }}^{0}(Y, X)$.

An important point is that $\operatorname{Corr}_{\text {mot }}^{0}(X, X)$ contains all Künneth components of the diagonal; see [5], Prop. 2.2. As a result we have a decomposition $\mathbf{H}(X)=$ $\oplus_{i \geq 0} \mathbf{H}^{i}(X)$ that in cohomological realizations gives the usual grading. Also, the Künneth projectors are used in giving the tensor product the correct commutativity constraint; see [5], 4.3. 
Let us summarize some basic properties of the category of motives thus obtained.

Properties 3.1.2. (i) The category $\operatorname{Mot}(K)$ is a $\mathbb{Q}$-linear graded semisimple Tannakian category.

(ii) If $\mathbf{M}$ is a motive over $K$, its endomorphism algebra $\operatorname{End}(\mathbf{M})$ is a finite-dimensional semisimple $\mathbb{Q}$-algebra that admits a positive involution $f \mapsto f^{\dagger}$. In particular, if $\mathbf{M}$ is a simple motive then $\operatorname{End}(\mathbf{M})$ is a division algebra with a positive involution and is therefore of the type classified by Albert; see for instance [62], Section 21.

(iii) Each classical cohomology theory $\mathrm{H}$ (de Rham, $\ell$-adic, Betti) gives rise to a realization functor $\mathrm{H}_{\text {mot }}$ : $\operatorname{Mot}(K) \rightarrow \operatorname{Vec}_{\mathrm{Q}}$ (with $\mathrm{Q}$ the coefficient field of $\mathrm{H}$ ) such that $\mathrm{H}=\mathrm{H}_{\text {mot }} \circ \mathbf{H}$. This functor $\mathrm{H}_{\text {mot }}$ is a Q-linear fibre functor of $\operatorname{Mot}(K)$.

To refer to the grading on $\operatorname{Mot}(K)$ one says weight rather than degree. For instance, for $X$ in $\operatorname{SmPr}(K)$ the motive $\mathbf{H}^{i}(X)(n)$ is pure of weight $i-2 n$.

Definition 3.1.3. The category of Artin motives is the full Tannakian subcategory $\operatorname{Mot}^{\operatorname{Art}}(K) \subset \operatorname{Mot}(K)$ generated by all motives $\operatorname{Spec}(L)$, for $K \subset L$ a finite (separable) field extension. The category of Abelian motives is the full Tannakian subcategory $\operatorname{Mot}^{\mathrm{Ab}}(K) \subset \operatorname{Mot}(K)$ generated by all Artin motives together with all motives of abelian varieties over $K$.

Motivic Galois groups. As we will discuss now, point 3.1.2(iii) leads to the introduction of motivic Galois groups. These will be important for us because of their (conjectural) relation with Mumford-Tate groups and the $\ell$-adic algebraic Galois groups $G_{\ell}^{0}$ that we have discussed in the previous sections. As before, let $\mathrm{H}$ be one of the classical cohomology theories, and let $\mathrm{Q}$ be the coefficient field. (More generally we could work with any Weil cohomology that gives the same notion of homological equivalence and in which the hard Lefschetz theorem holds.) In what follows we will simply write $\mathrm{H}$ for the realization functor $\mathrm{H}_{\text {mot }}$ on motives; this should not lead to confusion.

Let $\operatorname{Mot}(K)_{\mathrm{Q}}=\operatorname{Mot}(K) \otimes \mathrm{Q}$ be the $\mathrm{Q}$-linear extension of $\operatorname{Mot}(K)$, i.e., the category of $\mathrm{Q}$-modules in $\operatorname{Mot}(K)$. The objects of $\operatorname{Mot}(K)_{\mathrm{Q}}$ can be described as triples $\mathbf{M}=(X, e, n)$ where now $e$ is a projector in $\operatorname{Corr}_{\text {mot }}^{0}(X, X) \otimes \mathbf{Q}=A_{\operatorname{mot}}^{\operatorname{dim}(X)}\left(X \times_{K}\right.$ $X) \otimes \mathrm{Q}$. We have $\mathrm{H}(\mathbf{M})=e \cdot \mathrm{H}(X)(n)$, the image of the endomorphism induced by $e$ on $\mathrm{H}(X)(n)$.

By 3.1.2(iii), we have a fibre functor $\mathrm{H}: \operatorname{Mot}(K)_{\mathrm{Q}} \rightarrow \mathrm{Vec}_{\mathrm{Q}}$. We define the associated motivic Galois group by

$$
\mathscr{G}_{\mathrm{mot}, K, \mathrm{H}}=\operatorname{Aut}^{\otimes}(\mathrm{H}) \text {. }
$$

This is a pro-reductive group over Q. By Tannaka duality we have an equivalence of tensor categories between $\operatorname{Mot}(K)_{\mathrm{Q}}$ and the category $\operatorname{Rep}\left(\mathscr{G}_{\mathrm{mot}, K, \mathrm{H}}\right)$ of Q-linear finite dimensional representations of $\mathscr{G}_{\mathrm{mot}, K, \mathrm{H}}$. 
If $\mathbf{M} \in \operatorname{Mot}(K)_{\mathbf{Q}}$ is a motive with coefficients in $\mathrm{Q}$ and $\langle\mathbf{M}\rangle \subset \operatorname{Mot}(K)_{\mathbf{Q}}$ is the Tannakian subcategory generated by $\mathbf{M}$, we denote by

$$
G_{\text {mot }, \mathrm{H}}(\mathbf{M}) \subset \mathrm{GL}(\mathrm{H}(\mathbf{M}))
$$

the associated motivic Galois group. It can be defined as the automorphism group of $\mathrm{H}$ restricted to $\langle\mathbf{M}\rangle$. Alternatively, the motive $\mathbf{M}$ can be viewed as a representation $\mathscr{G}_{\mathrm{mot}, K, \mathrm{H}} \rightarrow \mathrm{GL}(\mathrm{H}(\mathbf{M}))$, and $G_{\text {mot }, \mathrm{H}}(\mathbf{M})$ is the algebraic group obtained as the image of this homomorphism. The group $G_{\text {mot, }}(\mathbf{M})$ is a (possibly non-connected) reductive group over $\mathrm{Q}$, and $\langle\mathbf{M}\rangle$ is equivalent to $\operatorname{Rep}\left(G_{\mathrm{mot}, \mathrm{H}}(\mathbf{M})\right)$ as a tensor category.

The fact that $\operatorname{Mot}(K)_{\mathrm{Q}}$ is neutral Tannakian means that we can think of motives in two very different ways: as triples $(X, e, n)$, or as algebraic representations of the motivic Galois group. The latter point of view, though much less geometric, turns out to be very useful.

Definition 3.1.4. Let $\mathrm{H}$ be a cohomology theory as above, and let $\mathbf{M}=(X, e, n)$ be an object of $\operatorname{Mot}(K)_{Q}$.

(i) An element $\xi \in \mathrm{H}(\mathbf{M})=e \cdot \mathrm{H}(X)(n)$ is a motivated cycle (with coefficients in $\mathrm{Q})$ if $\xi=e \cdot \psi$ for some $\psi \in A_{\text {mot }}^{n}(X) \otimes \mathrm{Q} \subset \mathrm{H}^{2 n}(X)(n)$.

(ii) A Q-linear subspace $\mathrm{H}^{\prime} \subset \mathrm{H}(\mathbf{M})$ is said to be motivated if $\mathrm{H}^{\prime}=\mathrm{H}(\mathbf{N})$ for some submotive $\mathbf{N} \subset \mathbf{M}$ in $\operatorname{Mot}(K)_{\mathbf{Q}}$.

The motivated cycles in $\mathrm{H}(\mathbf{M})$ are precisely the invariants in $\mathrm{H}(\mathbf{M})$ under the action of $G_{\mathrm{mot}, \mathrm{H}}(\mathbf{M})$. Note that for $\mathbf{M}=\mathbf{H}^{2 n}(X)(n)$ we recover the space $A_{\text {mot }}^{n}(X) \otimes$ $\mathrm{Q} \subset \mathrm{H}^{2 n}(X)(n)$. To say that $\mathrm{H}^{\prime} \subset \mathrm{H}(\mathbf{M})$ is motivated means precisely that $\mathrm{H}^{\prime}$ is stable under the action of $G_{\mathrm{mot}, \mathrm{H}}(\mathbf{M})$.

Just as the Mumford-Tate group of a polarizable $\mathbb{Q}$-Hodge structure is characterized by the fact that its tensor invariants are the Hodge classes (see Remark 2.1.8), the motivic Galois group $G_{\text {mot }}(\mathbf{M})$ is characterized by the fact that its tensor invariants are the motivated cycles.

Remark 3.1.5. One of Grothendieck's standard conjectures, called Conjecture B, states that, for $L$ an ample class on a smooth variety $X$, the Lefschetz involution $*_{L}$ is given by an algebraic cycle; if this is true for all $(X, L)$, the motivated cycles are precisely the cohomology classes of algebraic cycles. Let us note that in characteristic 0, Conjecture B is known to imply all other standard conjectures; see [6], Corollaire 5.4.2.2.

Remark 3.1.6. Suppose $\omega_{1}:\langle\mathbf{M}\rangle \rightarrow \mathrm{Vec}_{\mathrm{Q}_{1}}$ and $\omega_{2}:\langle\mathbf{M}\rangle \rightarrow \mathrm{Vec}_{\mathrm{Q}_{2}}$ are fibre functors, with $\mathrm{Q}_{1}$ and $\mathrm{Q}_{2}$ fields of characteristic 0 . The associated motivic Galois groups $G_{\text {mot }, i}(\mathbf{M})=\operatorname{Aut}^{\otimes}\left(\omega_{i}\right)$ are then inner forms of each other. We shall mostly work in a situation where $\omega_{1}$ and $\omega_{2}$ are given by cohomology theories $\mathrm{H}_{i}(i=1,2)$ with values in $Q_{i}$-vector spaces, and where, over some common field extension $Q_{1} \subset$ $\Omega \supset \mathrm{Q}_{2}$, we have a comparison isomorphism $\mathrm{H}_{1} \otimes \Omega \stackrel{\sim}{\longrightarrow} \mathrm{H}_{2} \otimes \Omega$. The induced isomorphism $\mathrm{GL}\left(\mathrm{H}_{1}(\mathbf{M})\right) \otimes \Omega \sim \mathrm{GL}\left(\mathrm{H}_{2}(\mathbf{M})\right) \otimes \Omega$ then restricts to an isomorphism $G_{\text {mot }, \mathrm{H}_{1}}(\mathbf{M}) \otimes \Omega \stackrel{\sim}{\longrightarrow} G_{\text {mot }, \mathrm{H}_{2}}(\mathbf{M}) \otimes \Omega$. 
Behaviour under field extension. Let $\mathrm{H}$ be a cohomology theory on $\operatorname{Sm} \operatorname{Pr}_{\bar{K}}$ with coefficient field Q. To simplify notation, write $\mathscr{G}_{\text {mot }, K}$ for $\mathscr{G}_{\text {mot, }, \mathrm{H}}$. We then have a short exact sequence

$$
1 \longrightarrow \mathscr{G}_{\mathrm{mot}, \bar{K}} \stackrel{a}{\rightarrow} \mathscr{G}_{\mathrm{mot}, K} \stackrel{b}{\rightarrow} \Gamma_{K} \longrightarrow 1,
$$

where, as before, $\Gamma_{K}=\operatorname{Gal}(\bar{K} / K)$. See for instance [37], Section 6. On the corresponding tensor categories, the maps $a$ and $b$ correspond to the base extension functor $\operatorname{Mot}(K)_{\mathrm{Q}} \rightarrow \operatorname{Mot}(\bar{K})_{\mathrm{Q}}$, respectively to the inclusion $\operatorname{Mot}^{\operatorname{Art}}(K)_{\mathrm{Q}} \hookrightarrow \operatorname{Mot}(K)_{\mathrm{Q}}$ of the subcategory of Artin motives.

On the other hand, for $\mathbf{M}$ in $\operatorname{Mot}(K)$ we have a surjective homomorphism $\mathscr{G}_{\text {mot }, K} \rightarrow G_{\text {mot }}(\mathbf{M})$. The image of the kernel of this homomorphism in $\Gamma_{K}$ is an open subgroup that corresponds to a finite Galois extension $K \subset K^{\diamond}(\mathbf{M})$, and we obtain a diagram with exact rows

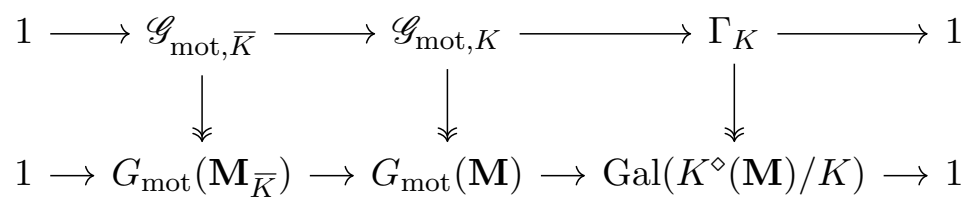

The homomorphism $G_{\text {mot }}\left(\mathbf{M}_{\bar{K}}\right) \rightarrow G_{\text {mot }}(\mathbf{M})$ is an isomorphism on identity components.

The extension $K \subset K^{\diamond}(\mathbf{M})$ may be characterized by its property that, for $K \subset$ $L \subset \bar{K}$, the (injective) homomorphism $G_{\mathrm{mot}}\left(\mathbf{M}_{\bar{K}}\right) \rightarrow G_{\mathrm{mot}}\left(\mathbf{M}_{L}\right)$ is an isomorphism if and only if $K^{\diamond}(\mathbf{M}) \subset L$.

The extension $K \subset K^{\diamond}(\mathbf{M})$ is independent of the chosen cohomology theory. In fact, to test whether $G_{\text {mot }}\left(\mathbf{M}_{\bar{K}}\right) \rightarrow G_{\text {mot }}\left(\mathbf{M}_{L}\right)$ is an isomorphism we may extend scalars (in the coefficient field, not the base field) to an overfield $\mathrm{Q} \subset \Omega$ and the claim follows using Remark 3.1.6. Conjecturally, $G_{\text {mot }}\left(\mathbf{M}_{\bar{K}}\right)$ is connected, and hence is the identity component of $G_{\text {mot }}(\mathbf{M})$. (Assuming $K$ to be finitely generated over $\mathbb{Q}$, this follows from Conjecture 3.2.2 below.) This is not known to be true, however. (Caution: the proof that is given in [37], Proposition 6.22 is incorrect.) See [49] for some partial results in this direction. If indeed $G_{\text {mot }}\left(\mathbf{M}_{\bar{K}}\right)$ were connected, it would be natural to write $K^{\text {conn }}(\mathbf{M})$ instead of $K^{\diamond}(\mathbf{M})$, in analogy with the notation $K^{\text {conn }}(\rho)$ that was introduced in Remark 2.2.2(ii).

Remark 3.1.7. Let $\bar{K} \subset \bar{L}$ be an extension of algebraically closed fields of characteristic 0 , and for $\mathrm{H}$ let us take étale cohomology with $\mathbb{Q}_{\ell}$-coefficients. If $\mathbf{M}$ is a motive over $\bar{K}$, we have a canonical isomorphism $G_{\text {mot }}\left(\mathbf{M}_{\bar{L}}\right) \stackrel{\sim}{\longrightarrow} G_{\operatorname{mot}}(\mathbf{M})$. This follows from the Scolie in [5], Section 2.5, together with the fact that for any $X$ in $\operatorname{SmPr}(\bar{K})$ we have $\mathrm{H}_{\text {ét }}\left(X, \mathbb{Q}_{\ell}\right) \stackrel{\sim}{\longrightarrow} \mathrm{H}_{\text {ét }}\left(X_{\bar{L}}, \mathbb{Q}_{\ell}\right)$.

\subsection{The motivated Mumford-Tate conjecture}

The title of this section is slightly misleading, in that we will not discuss one single conjecture but rather a package of several conjectures that naturally fit together. Throughout the discussion, $K$ denotes a finitely generated field extension of $\mathbb{Q}$. We fix an algebraic closure $K \subset \bar{K}$ and write $\Gamma_{K}=\operatorname{Gal}(\bar{K} / K)$. 
Betti and $\ell$-adic realization functors. For every complex embedding $\sigma: K \rightarrow \mathbb{C}$ we have an exact faithful tensor functor $\mathrm{H}_{\sigma}: \operatorname{Mot}(K) \rightarrow \mathrm{HS}_{\mathbb{Q}}^{\text {pol }}$, sending a motive $\mathbf{M}=(X, e, n)$ to the polarizable Hodge structure $e \cdot \mathrm{H}\left(X_{\sigma}, \mathbb{Q}\right)(n)$. Composing this with the forgetful functor we obtain a fibre functor $\operatorname{Mot}(K) \rightarrow \operatorname{Vec}_{\mathbb{Q}}$. We again denote the latter by $\mathrm{H}_{\sigma}$; the context will make it clear whether by $\mathrm{H}_{\sigma}(\mathbf{M})$ we mean the $\mathbb{Q}$-Hodge structure or the underlying $\mathbb{Q}$-vector space.

Associated with $\mathrm{H}_{\sigma}$ we have a motivic Galois group $\mathscr{G}_{\text {mot, } \mathrm{H}_{\sigma}}$ for which we use the simpler notation $\mathscr{G}_{\text {mot, } \sigma}$. For $\mathbf{M}$ in $\operatorname{Mot}(K)$ we denote by

$$
G_{\text {mot }, \sigma}(\mathbf{M}) \subset \mathrm{GL}\left(\mathrm{H}_{\sigma}(\mathbf{M})\right)
$$

the motivic Galois group of the Tannakian subcategory $\langle\mathbf{M}\rangle \subset \operatorname{Mot}(K)$.

Let $\mathbf{M}_{\sigma}$ denote the motive over $\mathbb{C}$ obtained from $\mathbf{M}$ by extension of scalars via $\sigma$. On motives over $\mathbb{C}$ singular cohomology with $\mathbb{Q}$-coefficients gives a fibre functor, and we write $G_{\text {mot,B }}\left(\mathbf{M}_{\sigma}\right)$ (with B for "Betti") for the associated motivic Galois group of $\mathbf{M}_{\sigma}$. If $\tilde{\sigma}: \bar{K} \rightarrow \mathbb{C}$ is an embedding with $\left.\tilde{\sigma}\right|_{K}=\sigma$, we have $G_{\operatorname{mot}, \tilde{\sigma}}\left(\mathbf{M}_{\bar{K}}\right)=$ $G_{\text {mot,B }}\left(\mathbf{M}_{\sigma}\right)$ as subgroups of $\mathrm{GL}\left(\mathrm{H}_{\sigma}(\mathbf{M})\right)$; so $G_{\text {mot,B }}\left(\mathbf{M}_{\sigma}\right)$ is a union of connected components of $G_{\operatorname{mot}, \sigma}(\mathbf{M})$ and conjecturally it is only the identity component.

We write $\operatorname{MT}\left(\mathbf{M}_{\sigma}\right)$ for the Mumford-Tate group of $\mathrm{H}_{\sigma}(\mathbf{M})$. Using the fact that for every $\mathbf{N}$ in $\operatorname{Mot}(\mathbb{C})$ the motivated cycles in the Betti cohomology $\mathrm{H}_{\mathrm{B}}(\mathbf{N})$ are Hodge classes, it follows that we have inclusions

$$
\operatorname{MT}\left(\mathbf{M}_{\sigma}\right) \subset G_{\text {mot,B }}\left(\mathbf{M}_{\sigma}\right) \subset \mathrm{GL}\left(\mathrm{H}_{\sigma}(\mathbf{M})\right) .
$$

Similarly, for every prime number $\ell$ we have an exact faithful tensor functor $\mathrm{H}_{\ell}: \operatorname{Mot}(K) \rightarrow \operatorname{Rep}\left(\Gamma_{K}\right)_{\mathbb{Q}_{\ell}}$, sending a motive $\mathbf{M}=(X, e, n)$ to the Galois representation $\rho_{\ell, \mathbf{M}}$ on $e \cdot \mathrm{H}\left(X_{\bar{K}}, \mathbb{Q}_{\ell}(n)\right)$. Composing this with the forgetful functor we obtain a fibre functor $\operatorname{Mot}(K) \rightarrow \operatorname{Vec}_{\mathbb{Q}}$ that we again denote by $\mathrm{H}_{\ell}$.

We write $\mathscr{G}_{\text {mot, } K, \ell}$ for the associated motivic Galois group of $\operatorname{Mot}(K)_{\mathbb{Q}_{\ell}}$, and for $\mathbf{M}$ in $\operatorname{Mot}(K)$ we denote by

$$
G_{\text {mot }, \ell}(\mathbf{M}) \subset \mathrm{GL}\left(\mathrm{H}_{\ell}(\mathbf{M})\right)
$$

the motivic Galois group of the Tannakian subcategory $\langle\mathbf{M}\rangle_{\mathbb{Q}_{\ell}} \subset \operatorname{Mot}(K)_{\mathbb{Q}_{\ell}}$. Further, $G_{\ell}(\mathbf{M})$ denotes the Zariski closure of the image of $\rho_{\ell, \mathbf{M}}$. From the fact that for every $\mathbf{N}$ in $\operatorname{Mot}(K)$ the motivated cycles in $\mathrm{H}_{\ell}(\mathbf{N})$ are Tate classes, it follows that we have inclusions

$$
G_{\ell}^{0}(\mathbf{M}) \subset G_{\text {mot }, \ell}\left(\mathbf{M}_{\bar{K}}\right) \subset \mathrm{GL}\left(\mathrm{H}_{\ell}(\mathbf{M})\right) .
$$

(Here we use that the inclusion $G_{\text {mot }, \ell}\left(\mathbf{M}_{\bar{K}}\right) \hookrightarrow G_{\text {mot }, \ell}(\mathbf{M})$ is an isomorphism on identity components, and that $G_{\operatorname{mot}, \ell}\left(\mathbf{M}_{\bar{K}}\right)$ is reductive.)

Remark 3.2.1. As already mentioned in Remark 2.2.10, the collection of $\ell$-adic representations $\left\{\rho_{\ell, \mathbf{M}}\right\}_{\ell}$ is not, in general, known to be a strictly compatible system of Galois representations. (If $\mathbf{M}=\mathbf{H}^{n}(X)$ for some smooth projective $X$, this is known.) This means we cannot apply the general results mentioned in 2.2.10. For instance, to the author's knowledge it is not known whether the field extension $K \subset K^{\text {conn }}\left(\rho_{\ell, \mathbf{M}}\right)$ (see Remark 2.2.2(ii)) is independent of $\ell$. Note, however, that if 
$\mathbf{M}=(X, e, n)$ then each $K^{\operatorname{conn}}\left(\rho_{\ell, \mathbf{M}}\right)$ is a subfield of $K^{\operatorname{conn}}\left(\rho_{\ell, \mathbf{H}(X)}\right)$, and the latter is a finite Galois extension of $K$ that by by a result of Serre (see [81] or [51], Proposition 6.14) is independent of $\ell$.

On the other hand, if by $\mathscr{G}_{K, \ell}$ we denote the projective limit of all $G_{\ell}(\mathbf{M})$ for $\mathbf{M}$ in $\operatorname{Mot}(K)$ then we have a diagram with exact rows

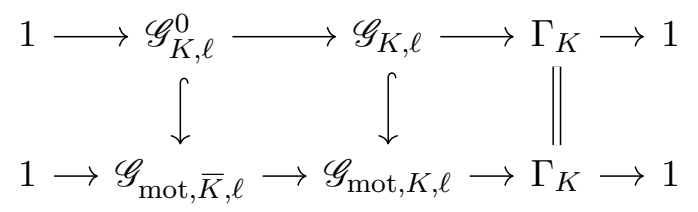

(The top row is in fact split.) This implies that the extension $K \subset K^{\diamond}(\mathbf{M})$ introduced at the end of Section 3.1, which is independent of $\ell$, is contained in $K^{\operatorname{conn}}\left(\rho_{\ell, \mathbf{M}}\right)$ for all $\ell$. Hence if $K=K^{\text {conn }}\left(\rho_{\ell, \mathbf{M}}\right)$ for some $\ell$ then $G_{\text {mot }}\left(\mathbf{M}_{\bar{K}}\right)=G_{\text {mot }}(\mathbf{M})$.

The Betti and $\ell$-adic realization functors are related via comparison isomorphisms. Let $\tilde{\sigma}: \bar{K} \rightarrow \mathbb{C}$ be a complex embedding, $\sigma$ its restriction to $K$, and let $\ell$ be a prime number. For $X / K$ smooth projective we have comparison isomorphisms $\mathrm{H}_{\mathrm{B}}\left(X_{\sigma}, \mathbb{Q}\right) \otimes \mathbb{Q}_{\ell} \sim \mathrm{H}_{\text {ét }}\left(X_{\sigma}, \mathbb{Q}_{\ell}\right) \stackrel{\sim}{\longrightarrow} \mathrm{H}_{\text {ét }}\left(X_{\bar{K}}, \mathbb{Q}_{\ell}\right)$. This gives rise to an isomorphism of fibre functors on $\operatorname{Mot}(K)_{\mathbb{Q}_{\ell}}$,

$$
I_{\tilde{\sigma}, \ell}: \mathrm{H}_{\sigma} \otimes \mathbb{Q}_{\ell} \stackrel{\sim}{\longrightarrow} \mathrm{H}_{\ell} .
$$

For $\mathbf{M}$ a motive over $K$ the induced isomorphism $\mathrm{GL}\left(\mathrm{H}_{\sigma}(\mathbf{M})\right) \otimes \mathbb{Q}_{\ell} \sim \mathrm{GL}_{(}\left(\mathrm{H}_{\ell}(\mathbf{M})\right)$ restricts to an isomorphism

$$
\gamma_{\tilde{\sigma}, \ell}: G_{\operatorname{mot}, \sigma}(\mathbf{M}) \otimes \mathbb{Q}_{\ell} \stackrel{\sim}{\longrightarrow} G_{\operatorname{mot}, \ell}(\mathbf{M}) .
$$

Conjecture 3.2.2 (Hodge classes are motivated). Let $\mathbf{M}$ be a motive over $K$. Let $\sigma$ be a complex embedding of $K$. Then the first inclusion in (3.1) is an equality, i.e.,

$$
\operatorname{MT}\left(\mathbf{M}_{\sigma}\right) \stackrel{?}{=} G_{\operatorname{mot}, \mathrm{B}}\left(\mathbf{M}_{\sigma}\right) \text {. }
$$

This conjecture may of course be stated directly for a motive over $\mathbb{C}$, without any choice of a model over a finitely generated field. Our choice to start with an $\mathbf{M}$ over $K$ is motivated (no pun intended) by the desire to show the similarities with other conjectures, such as the next.

Conjecture 3.2.3 (Tate classes are motivated). Let $\mathbf{M}$ be a motive over $K$. Let $\ell$ be a prime number. Then the first inclusion in (3.2) is an equality, i.e.,

$$
G_{\ell}^{0}(\mathbf{M}) \stackrel{?}{=} G_{\operatorname{mot}, \ell}\left(\mathbf{M}_{\bar{K}}\right) \text {. }
$$

Conjecture $\mathrm{HM}_{\sigma}$ is equivalent to the assertion that for every motive $\mathbf{N}$ in $\left\langle\mathbf{M}_{\sigma}\right\rangle \subset \operatorname{Mot}(\mathbb{C})$, all Hodge classes in its Betti realization $\mathrm{H}_{\mathrm{B}}(\mathbf{N})$ are motivated cycles. Hence, if $\mathbf{M}=(X, e, n)$ and if the Hodge conjecture is true for all powers of $X_{\sigma}$, Conjecture $\mathrm{HM}_{\sigma}$ is true for M. Similarly, Conjecture $\mathrm{TM}_{\ell}$ is equivalent to the assertion that the Galois representation $\rho_{\ell, \mathbf{M}}$ is semisimple and that every Tate class in $\mathrm{H}_{\ell}(\mathbf{N})$, for $\mathbf{N}$ in $\left\langle\mathbf{M}_{L}\right\rangle \subset \operatorname{Mot}(L)$ with $K \subset L \subset \bar{K}$, is a motivated cycle 
over $\bar{K}$. Hence, if $\mathbf{M}=(X, e, n)$ and if the Tate conjecture is true for all powers of $X$, Conjecture $\mathrm{TM}_{\ell}$ is true for $\mathbf{M}$.

Conjectures $\mathrm{HM}_{\sigma}$ and $\mathrm{TM}_{\ell}$ each imply that $G_{\text {mot, } \ell}\left(\mathbf{M}_{\bar{K}}\right)$ is connected. It can be shown that $\mathrm{TM}_{\ell}$ also implies that $G_{\ell}(\mathbf{M})$ is equal to $G_{\operatorname{mot}, \ell}(\mathbf{M})$. (Use the Scolie in [5], Section 2.5.)

Conjecture 3.2.4 (Mumford-Tate conjecture). Let $\mathbf{M}$ be a motive over $K$. Let $\tilde{\sigma}$ be a complex embedding of $\bar{K}$, and let $\ell$ be a prime number. Then the isomorphism $\mathrm{GL}\left(\mathrm{H}_{\sigma}(\mathbf{M})\right) \otimes \mathbb{Q}_{\ell} \stackrel{\sim}{\sim} \mathrm{GL}\left(\mathrm{H}_{\ell}(\mathbf{M})\right)$ that is induced by the comparison isomorphism $I_{\tilde{\sigma}, \ell}$ restricts to an isomorphism

$$
\operatorname{MT}\left(\mathbf{M}_{\sigma}\right) \otimes \mathbb{Q}_{\ell} \stackrel{?}{\stackrel{?}{\longrightarrow}} G_{\ell}^{0}(\mathbf{M})
$$

Remarks 3.2.5. (i) If two of the above three conjectures are true then so is the third. (Recall that $G_{\mathrm{mot}, \mathrm{B}}\left(\mathbf{M}_{\sigma}\right)=G_{\mathrm{mot}, \tilde{\sigma}}\left(\mathbf{M}_{\bar{K}}\right)$ and that under the comparison isomorphism $I_{\tilde{\sigma}, \ell}$ we have $G_{\text {mot }, \tilde{\sigma}}\left(\mathbf{M}_{\bar{K}}\right) \otimes \mathbb{Q}_{\ell}=G_{\text {mot }, \ell}\left(\mathbf{M}_{\bar{K}}\right)$ as subgroups of $\operatorname{GL}\left(\mathrm{H}_{\ell}(\mathbf{M})\right)$.) By the motivic Mumford-Tate conjecture (for given $\tilde{\sigma}$ and $\ell$ ) we mean the conjunction of the three conjectures.

(ii) Of course, Conjectures 3.2.2 and 3.2.3 are implied by the Hodge Conjecture and the Tate Conjecture, respectively. One may split up the HC or TC into two parts: (a) Hodge classes (resp. Tate classes) are motivated, (b) Motivated cycles are algebraic. One can say some interesting things about (b); notably there is a result of Voisin [104] that if motivated cycles on (smooth projective) varieties over $\overline{\mathbb{Q}}$ are algebraic, the same is true on smooth projective varieties over $\mathbb{C}$. Voisin in fact works with absolute Hodge classes rather than motivated cycles, but the argument works in either setting. She also proves that if the HC is true for varieties over $\overline{\mathbb{Q}}$, the Hodge conjecture on complex varieties can be reduced to showing that certain Hodge loci are defined over $\overline{\mathbb{Q}}$. See also [103], Section 3 .

\subsection{Some known results (2)}

"Hodge classes are motivated" for abelian motives. One of the highlights in our present knowledge about the motivated Mumford-Tate conjecture, is the following result of Deligne and André.

Theorem 3.3.1. Let $\mathbf{M}$ be an abelian motive over $\mathbb{C}$. Then Conjecture 3.2 .2 is true for $\mathbf{M}$, i.e., $\mathrm{MT}(\mathbf{M})=G_{\mathrm{mot}, \mathrm{B}}(\mathbf{M})$.

This result was proven by Deligne [35], working in the category of motives for absolute Hodge cycles. André, who already simplified part of Deligne's proof in [2], proved the result for his category of motives in [5], Section 6. Let us also note that Deligne's result was extended to 1-motives by Brylinski (see [17], Théorème 2.2.5), and that it was strengthened to include a $p$-adic comparison property by Blasius, Ogus and Wintenberger (see [13] or [108] and the references contained therein). The latter strengthening also follows from André's version of the result.

Let $\mathbf{M}$ be an abelian motive over a finitely generated field $K$ of characteristic 0 . By Theorem 3.3.1, Conjecture $\mathrm{HM}_{\sigma}$ is then true for $\mathbf{M}$, for all $\sigma$; this implies that 
the Mumford-Tate Conjecture $\mathrm{MTC}_{\tilde{\sigma}, \ell}$ is equivalent to Conjecture $\mathrm{TM}_{\ell}$ and does not depend on $\tilde{\sigma}$. By Theorem 2.2.7 (the characteristic 0 case, proven by Faltings) we also know that $G_{\ell}^{0}(\mathbf{M})$ is reductive, as it is a quotient of the $G_{\ell}^{0}$ of an abelian variety. Moreover, the Deligne-André theorem has as obvious consequence that we get one of the inclusions predicted by the Mumford-Tate conjecture: with notation as in Conjecture 3.2.4 we have

$$
\operatorname{MT}\left(\mathbf{M}_{\sigma}\right) \otimes \mathbb{Q}_{\ell} \supset G_{\ell}^{0}(\mathbf{M})
$$

This result was also obtained by Piatetksi-Shapiro and Borovoi; see [15].

Another consequence is that for abelian motives the Mumford-Tate conjecture is true on connected centres. With notation as in Section 3.2, the precise statement is the following.

Theorem 3.3.2. Let $\mathbf{M}$ be an abelian motive over the finitely generated field $K$ of characteristic 0 . Choose a complex embedding $\tilde{\sigma}$ of $\bar{K}$ and a prime number $\ell$. Let $Z_{\sigma}$ be the centre of the Mumford-Tate group $\mathrm{MT}\left(\mathbf{M}_{\sigma}\right)$, and let $Z_{\ell}$ be the centre of the $\ell$-adic algebraic Galois group $G_{\ell}^{0}(\mathbf{M})$. Then under the isomorphism $\mathrm{GL}\left(\mathrm{H}_{\sigma}\right) \otimes \mathbb{Q}_{\ell} \sim$ $\mathrm{GL}\left(\mathrm{H}_{\ell}\right)$ induced by the comparison isomorphism $\mathrm{H}_{\sigma} \otimes \mathbb{Q}_{\ell} \sim \mathrm{H}_{\ell}$, the image of $Z_{\sigma} \otimes \mathbb{Q}_{\ell}$ contains $Z_{\ell}$ and we have an isomorphism $Z_{\sigma}^{0} \otimes \mathbb{Q}_{\ell} \stackrel{\sim}{\longrightarrow} Z_{\ell}^{0}$.

This result, which of course generalizes the result mentioned in 2.4.4, is due to Vasiu; see Vasiu [102], Theorem 1.3.1. A different proof is given by Ullmo and Yafaev in [98], Corollary 2.11. In these papers the result is stated only for abelian varieties, but it is easy to deduce from this the same conclusion for abelian motives.

Which motives are abelian? Consider motives over an algebraically closed field $K$ of characteristic 0 . It is clear from the definition of the category of abelian motives that any submotive of a product of curves and abelian varieties lies in this category. (Note that the $\mathbf{H}^{1}$ of a curve is isomorphic to the $\mathbf{H}^{1}$ of its Jacobian and hence lies in $\operatorname{Mot}^{\mathrm{Ab}}(K)$.) We refer to [73] for a beautiful study of how big this class is. Let us note, for instance, that ruled surfaces, unirational varieties and Fermat hypersurfaces are all dominated by products of curves. On the other hand, "most" motives do not lie in $\operatorname{Mot}^{\mathrm{Ab}}(K)$. As remarked by Deligne at the end of his paper [32], from the structure of the Mumford-Tate group one can sometimes see that a Hodge structure does not lie in the Tannakian subcategory of $\mathrm{HS}_{\mathbb{Q}}$ generated by all abelian varieties. (This observation lies at the basis of the results in [73].) For instance, for $n \geq 2$ the motive of a very general hypersurface $X \subset \mathbb{P}_{\mathbb{C}}^{n+1}$ of degree $\geq n+3$ is known not to lie in $\operatorname{Mot}^{\mathrm{Ab}}(\mathbb{C})$.

On the positive side, there are some non-trivial examples of abelian motives. For instance, [4], Theorem 1.5.1, contains as particular instances the fact that the motive of a complex K3 surface, and more generally the $\mathbf{H}^{2}$ of any complex hyperkähler variety with second Betti number $B_{2}>3$, lies in $\operatorname{Mot}^{\mathrm{Ab}}(\mathbb{C})$. We will say more about the results obtained in [4] in Section 4.4. 
Dependence on $\sigma$ and $\ell$. Let us now address the question how the conjectures stated in Section 3.2 depend on $\sigma$ (or $\tilde{\sigma}$ ) and $\ell$. The brief answer is that these conjectures are known to be independent of $\sigma$ and $\ell$ for abelian motives, and that for general motives not much seems known.

First let us consider the dependence on $\sigma$ or $\tilde{\sigma}$. As we have already seen, for abelian motives this is not an issue. For more general motives, the problem that we run into is the following. Suppose we have a nonsingular projective variety $Y$ over $\mathbb{C}$ and an automorphism $\gamma$ of $\mathbb{C}$. We may then form ${ }^{\gamma} Y$, the pull-back of $Y$ via $\operatorname{Spec}(\gamma): \operatorname{Spec}(\mathbb{C}) \rightarrow \operatorname{Spec}(\mathbb{C})$, and a morphism of schemes ${ }^{\gamma} Y \rightarrow Y$, which however is not a morphism of schemes over $\mathbb{C}$, unless $\gamma$ is the identity. As explained for instance in [25], on de Rham cohomology the latter map induces a $\gamma$-linear isomorphism $\mathrm{dR}(\gamma): \mathrm{H}_{\mathrm{dR}}(Y / \mathbb{C}) \stackrel{\sim}{\sim} \mathrm{H}_{\mathrm{dR}}\left({ }^{\gamma} Y / \mathbb{C}\right)$ that is compatible with the Hodge filtrations.

If we start with a motivated cycle $\alpha$ in $\mathrm{H}(Y, \mathbb{Q})$, and if we write $\alpha_{\mathrm{dR}}$ for its image in $\mathrm{H}_{\mathrm{dR}}(Y / \mathbb{C})$, then $\mathrm{dR}(\gamma)\left(\alpha_{\mathrm{dR}}\right)$ is again a motivated cycle, i.e., $\mathrm{dR}(\gamma)\left(\alpha_{\mathrm{dR}}\right)=\beta_{\mathrm{dR}}$ for a motivated cycle $\beta$ on ${ }^{\gamma} Y$. This property just means that motivated cycles are absolute Hodge classes. (Cf. [5], Proposition 2.5.1.)

Now suppose $Y=X_{\sigma}$ for $X$ in $\operatorname{SmPr}_{K}$ and $\sigma: K \rightarrow \mathbb{C}$, in which case ${ }^{\gamma} Y=X_{\tau}$ with $\tau=\gamma \circ \sigma$. If we assume that conjecture $\mathrm{HM}_{\sigma}$ is true for $X$ then all Hodge classes $\alpha$ on $X_{\sigma}$ are motivated cycles; hence, by the recipe just explained, they can be transported to motivated cycles on $X_{\tau}$. This gives a collection of Hodge classes on $X_{\tau}$ of which we know they are motivated. But a priori there could be more Hodge classes on $X_{\tau}$, which prevents us from concluding that $\mathrm{HM}_{\sigma}$ implies $\mathrm{HM}_{\tau}$. For Conjecture $\mathrm{MTC}_{\tilde{\sigma}, \ell}$ a similar problem occurs. (If $K$ is a number field, it is easy to see that $\mathrm{MTC}_{\tilde{\sigma}, \ell}$ only depends on $\sigma$ and not on the choice of an embedding $\tilde{\sigma}$ extending it.)

Next let us discuss the dependence of conjectures $\mathrm{TM}_{\ell}$ and $\mathrm{MTC}_{\tilde{\sigma}, \ell}$ on $\ell$. For abelian motives, it is known that these conjectures (which, as discussed above, for abelian motives are equivalent) are independent of $\ell$. This uses some facts that are not known for arbitrary motives. To explain what is going on, let us sketch the argument for a motive $\mathbf{M}=\mathbf{H}^{1}(X)$, where $X$ is an abelian variety. There are three key ingredients. (1) We know that the system of $\ell$-adic Galois representations $\left\{\rho_{\ell, X}\right\}$ is a strictly compatible system. (Cf. Remark 2.2.10.) By a result of Serre ([81], Section 3, or [51], Proposition 6.12) this implies that the rank of $G_{\ell}^{0}(X)$ is independent of $\ell$. (2) As discussed above, $G_{\ell}^{0}(X) \subset \mathrm{MT}\left(X_{\sigma}\right) \otimes \mathbb{Q}_{\ell}$. (3) As we have seen in Theorem 3.3.2, $Z_{\ell} \subset\left(Z_{\sigma} \otimes \mathbb{Q}_{\ell}\right)$. Now suppose the MTC is true for some $\ell$. Then by (1) and (2), for every $\ell$ the rank of $G_{\ell}^{0}(X)$ equals the rank of $\operatorname{MT}\left(X_{\sigma}\right)$, and by an application of the Borel-de Siebenthal theorem it follows from (3) that $G_{\ell}^{0}(X)=\operatorname{MT}\left(X_{\sigma}\right) \otimes \mathbb{Q}_{\ell}$. (A modern reference for the Borel-de Siebenthal theorem is $[65]$.

Even for motives of the form $\mathbf{M}=\mathbf{H}^{n}(X)$ with $X$ a nonsingular projective variety (for which (1) is known), attempts to generalize this get stuck on the fact that properties (2) and (3) are not known in general. If we restrict our attention to abelian motives $\mathbf{M}$ then (2) and (3) are still valid but property (1) is not known in general. 
Fortunately, Commelin [27] has been able to prove, for an abelian motive, that the system of Galois representations $\left\{\rho_{\ell, \mathbf{M}}\right\}$ satisfies a slightly weaker compatibility property, which suffices to conclude that the rank of $G_{\ell}^{0}(\mathbf{M})$ is independent of $\ell$. (There are related results of Laskar [53].) This gives the desired conclusion for abelian motives; see [28], Corollary 7.6.

Product situations. Suppose $H_{1}$ and $H_{2}$ are Hodge structures of which we know the Mumford-Tate groups. In general there is no easy recipe for the Mumford-Tate group of $H_{1} \oplus H_{2}$. As we have seen in 2.1.4(iii), $\mathrm{MT}\left(H_{1} \oplus H_{2}\right)$ is an algebraic subgroup of $\operatorname{MT}\left(H_{1}\right) \times \mathrm{MT}\left(H_{2}\right)$ that maps surjectively onto both factors, but only based on this information we cannot determine $\mathrm{MT}\left(H_{1} \oplus H_{2}\right)$. Of course, there can be reasons why this group has to be "small"; e.g., if there exists an isomorphism of Hodge structures $f: H_{1} \stackrel{\sim}{\longrightarrow} H_{2}$ then $\mathrm{MT}\left(H_{1} \oplus H_{2}\right)$ is the graph $\Gamma_{f} \subset \mathrm{MT}\left(H_{1}\right) \times \mathrm{MT}\left(H_{2}\right)$ of the induced isomorphism $\mathrm{MT}\left(H_{1}\right) \stackrel{\sim}{\longrightarrow} \mathrm{MT}\left(H_{2}\right)$. (Cf. 2.1.4(iv).) At the other extreme, suppose $X$ and $Y$ are abelian varieties such that all simple factors of the endomorphism algebra $\operatorname{End}^{0}(X)$ are of Albert Type I, II or III, and such that $Y$ is of CM type. Then the special Mumford-Tate group $\operatorname{SMT}(X)$ is semisimple (see 2.4.3), whereas $\operatorname{SMT}(Y)$ is a torus; using this one finds that $\operatorname{SMT}(X \times Y)=\operatorname{SMT}(X) \times \operatorname{SMT}(Y)$. (We have to use special Mumford-Tate groups here: as discussed in Remark 2.1.12 we have $\mathrm{MT}=\left(\mathbb{G}_{\mathrm{m}} \cdot \mathrm{id}\right) \cdot \mathrm{SMT}$ but $\mathrm{MT}(X \times Y)$ does not contain $\left(\mathbb{G}_{\mathrm{m}} \cdot \mathrm{id}\right) \times\left(\mathbb{G}_{\mathrm{m}} \cdot \mathrm{id}\right)$. $)$

For $\ell$-adic algebraic Galois groups and motivic Galois groups, analogous remarks can be made. This has as consequence that if we know one of the conjectures 3.2.23.2.4 for motives $\mathbf{M}$ and $\mathbf{N}$, in general there is no easy way to deduce that same conjecture for $\mathbf{M} \oplus \mathbf{N}$.

For abelian varieties, under additional hypotheses more can be said. We refer the reader to [42], [69], [54], [59] and the references contained therein, and also to [102], Theorem 1.3.7. As an example of what comes out, let us mention that the HC, TC and MTC are true for any complex abelian variety whose simple factors all have dimension $\leq 2$. Commelin [27] recently proved the Mumford-Tate conjecture for any product of abelian motives of K3 type. It is hoped that his methods can be extended to handle many more product situations, assuming the MTC for the factors.

\section{Behaviour in families}

\subsection{Variation of Hodge structure}

Algebraic monodromy groups. Let $S$ be an irreducible nonsingular complex algebraic variety. We denote by $S^{\text {an }}$ the associated complex manifold. If $\mathrm{Q}$ is a coefficient field of characteristic 0 , let $\mathrm{LS}\left(S^{\mathrm{an}}\right)_{\mathrm{Q}}$ denote the category of local systems of Q-vector spaces on $S^{\text {an }}$. There is an obvious tensor product on $\operatorname{LS}\left(S^{\text {an }}\right)_{\mathrm{Q}}$, making it a Q-linear neutral Tannakian category. If $b \in S$ is a base point, the functor $\mathrm{Fib}_{b}: \operatorname{LS}\left(S^{\mathrm{an}}\right)_{\mathrm{Q}} \rightarrow \operatorname{Vec}_{\mathrm{Q}}$ given by sending a local system $\mathscr{V}$ to its fibre $V=\mathscr{V}_{b}$ at $b$ 
is a fibre functor. We have a monodromy representation

$$
\rho=\rho_{\mathscr{V}}: \pi_{1}\left(S^{\mathrm{an}}, b\right) \rightarrow \mathrm{GL}(V)
$$

that completely determines $\mathscr{V}$. The automorphism group of the fibre functor $\mathrm{Fib}_{b}$ on the Tannakian subcategory $\langle\mathscr{V}\rangle \subset \mathrm{LS}\left(S^{\mathrm{an}}\right)_{\mathrm{Q}}$ generated by $\mathscr{V}$ is the algebraic monodromy group

$$
G_{\text {mono }}(\mathscr{V} / S) \subset \mathrm{GL}(V)
$$

obtained as the Zariski closure of the image of $\rho$. This is an algebraic group over $\mathbf{Q}$. By Tannakian theory we have an equivalence of tensor categories between $\langle\mathscr{V}\rangle$ and the category $\operatorname{Rep}\left(G_{\text {mono }}(\mathscr{V} / S)\right.$ ) of (finite dimensional, algebraic) representations of $G_{\text {mono }}(\mathscr{V} / S)$ over Q.

Let us also recall that $\operatorname{LS}\left(S^{\text {an }}\right)_{\mathbb{C}}$ is equivalent to the category $\operatorname{MIC}\left(S^{\text {an }}\right)$ of flat holomorphic vector bundles $(\mathscr{V}, \nabla)$ on $S^{\text {an }}$. (MIC is for module with integrable connection.) The latter, in turn, is equivalent to the category $\operatorname{MIC}^{\mathrm{reg}}(S)$ of algebraic flat vector bundles with regular singularities; see [30], Théorème II.5.9.

The algebraic monodromy group $G_{\text {mono }}(\mathscr{V} / S)$ is not connected, in general. If $f: S^{\prime} \rightarrow S$ is a generically finite dominant morphism, the algebraic monodromy group of $f^{*}(\mathscr{V})$ over $S^{\prime}$ may be smaller than $G_{\text {mono }}(\mathscr{V} / S)$ but the two have the same identity component. The inverse image of the identity component $G_{\text {mono }}^{0}(\mathscr{V} / S) \subset$ $G_{\text {mono }}(\mathscr{V} / S)$ in $\pi_{1}(S, b)$ corresponds to a connected étale cover $\nu: S^{\operatorname{conn}}(\mathscr{V}) \rightarrow S$ such that the algebraic monodromy group of $\nu^{*}(\mathscr{V})$ is connected. This cover plays a role analogous to the field extension $K \subset K^{\text {conn }}(\rho)$ associated with a Galois representation $\rho$.

Remark 4.1.1. In what follows we will sometimes consider local systems of algebraic groups $G \subset \operatorname{GL}(\mathscr{V})$. If $b$ is a base point and $V=\mathscr{V}_{b}$, the fibre $G_{b} \subset \mathrm{GL}(V)$ is an algebraic subgroup that is normalized by $G_{\text {mono }}(\mathscr{V} / S)$ (or, what is equivalent, by the action of $\left.\pi_{1}\left(S^{\text {an }}, b\right)\right)$. Conversely, if we have an algebraic subgroup $G_{b} \subset \mathrm{GL}(V)$ that is normalized by $G_{\text {mono }}(\mathscr{V} / S)$, it is the fibre at $b$ of a uniquely determined local system of algebraic groups $G \subset \mathrm{GL}(\mathscr{V})$. We may, for instance, view the algebraic monodromy group itself as a local system $G_{\text {mono }}(\mathscr{V} / S) \subset \mathrm{GL}(\mathscr{V})$ whose fibre at any point $s$ is the image of $\pi_{1}\left(S^{\text {an }}, s\right)$ in its monodromy representation on $\mathscr{V}_{s}$.

The generic Mumford-Tate group of a VHS. Let us recall that, with $S$ as above, a $\mathbb{Q}$-variation of Hodge structure (abbreviated VHS) of weight $n$ over $S$ is given by a $\mathbb{Q}$-local system $\mathscr{V}$ on $S^{\text {an }}$ together with a finite descending filtration Fil of $\mathscr{V} \otimes_{\mathbb{Q}} \mathscr{O}_{S}$ by holomorphic subbundles such that:

- for every $s \in S$, the filtration $\mathrm{Fil}_{s}^{\bullet}$ on the fibre $\mathscr{V}_{s} \otimes \mathbb{C}$ defines a Hodge structure of weight $n$;

- for all indices $i$ we have $\nabla\left(\mathrm{Fil}^{i}\right) \subset \Omega_{S}^{1} \otimes \mathrm{Fil}^{i-1}$ (Griffiths transversality).

We refer to [66], Chapter 10, for a much more detailed discussion. In what follows we denote by $\overline{\mathscr{V}}$ the local system underlying the VHS $\mathscr{V}$. We denote the category of $\mathbb{Q}$-VHS over $S$ by $\operatorname{VHS}(S)_{\mathbb{Q}}$. As in the case of pointwise Hodge structures, we have Tate twists and the notion of a polarization. All variations that are of interest 
to us are polarizable, and the subcategory $\operatorname{VHS}^{\text {pol }}(S)_{\mathbb{Q}}$ of polarizable variations is semisimple. There is an obvious tensor product on $\operatorname{VHS}^{\text {pol }}(S)_{\mathbb{Q}}$, making it a neutral $\mathbb{Q}$-linear Tannakian category. The functor $\mathrm{Fib}_{b}$ given by $\mathscr{V} \mapsto \mathscr{V}_{b}$ is again a fibre functor. We shall describe the algebraic group corresponding with $\langle\mathscr{V}\rangle \subset \operatorname{VHS}^{\text {pol }}(S)_{\mathbb{Q}}$ after Theorem 4.1.3 below.

For the purpose of this paper we mostly care about variations of Hodge structure $\mathscr{V}$ with $\mathbb{Q}$-coefficients. There are some results, however, in which it is important that $\mathscr{V}$ comes from a variation with $\mathbb{Z}$-coefficients. For $b \in S$ a base point and $V=\mathscr{V}_{b}$, this is equivalent to the condition that there is a lattice $V_{\mathbb{Z}} \subset V$ that is stable under the action of $\pi_{1}\left(S^{\mathrm{an}}, b\right)$.

If $\mathscr{V}$ is a $\mathbb{Q}$-VHS over $S$ then for every $s \in S$ we have a Hodge structure on the fibre $\mathscr{V}_{s}$ and an associated Mumford-Tate group $\operatorname{MT}\left(\mathscr{V}_{s}\right) \subset \operatorname{GL}\left(\mathscr{V}_{s}\right)$. The following result gives important information on how this Mumford-Tate group varies with $s$.

Theorem 4.1.2. Let $S$ be an irreducible nonsingular complex algebraic variety. Let $\mathscr{V}$ be a polarizable $\mathbb{Q}$-VHS on $S$ that admits a $\mathbb{Z}$-structure, and denote by $\overline{\mathcal{V}}$ the underlying $\mathbb{Q}$-local system. Then there exist a local system of algebraic subgroups $M=\operatorname{MT}(\mathscr{V} / S) \subset \operatorname{GL}(\overline{\mathscr{V}})$ and a subset $\operatorname{Exc}(\mathscr{V}) \subset S$ that is a countable union of algebraic subvarieties $S^{\prime} \subsetneq S$, such that:

(a) for every $s \in S$ we have $\operatorname{MT}\left(\mathscr{V}_{s}\right) \subset M_{s}$;

(b) the inclusion $\operatorname{MT}\left(\mathscr{V}_{s}\right) \subset M_{s}$ is strict if and only if $s \in \operatorname{Exc}(\mathscr{V})$.

The points in $\operatorname{Hgen}(\mathscr{V})=S \backslash \operatorname{Exc}(\mathscr{V})$ are called the Hodge-generic points for the variation $\mathscr{V}$. If the context requires it, we write $\operatorname{Hgen}(\mathscr{V} / S)$. By definition, $\operatorname{MT}(\mathscr{V} / S)_{s}=\operatorname{MT}\left(\mathscr{V}_{s}\right)$ for all Hodge-generic points. We call $\operatorname{MT}(\mathscr{V} / S)$ the generic Mumford-Tate group of $\mathscr{V}$. In practice, we often choose a base point $b \in S$ and work with the fibre $\operatorname{MT}(\mathscr{V} / S)_{b} \subset \mathrm{GL}(V)$, where $V=\mathscr{V}$. By Remark 4.1.1, this fibre completely determines $\operatorname{MT}(\mathscr{V} / S)$. We again use the notation $\operatorname{MT}(\mathscr{V} / S)$ for this fibre.

It is easiest to describe the exceptional locus by working in a situation where the underlying local system $\overline{\mathscr{V}}$ is trivialized. As Mumford-Tate groups control tensor invariants, we need to consider not only $\mathscr{V}$ but also all tensor spaces $\mathscr{W}=T(\mathscr{V})$ built from it. If $u: \tilde{S} \rightarrow S$ is the universal cover and $\tilde{b} \in \tilde{S}$ lies above $b \in S$, we have a trivialization $u^{*} \overline{\mathcal{V}} \cong V \times \tilde{S}$, with $V=\mathscr{V}_{b}$. Likewise, for any tensor construction $\mathscr{W}$ we have $u^{*} \overline{\mathscr{W}} \cong W \times \tilde{S}$, with $W=\mathscr{W}_{b}=T(V)$. For every $\tilde{s} \in \tilde{S}$ we have a Hodge structure on $W \times\{\tilde{s}\}$. Any $\xi(\tilde{s}) \in W \times\{\tilde{s}\}$ uniquely extends to a section $\xi$ over $\tilde{S}$. Given such $\xi \in \Gamma\left(\tilde{S}, u^{*} \overline{\mathscr{W}}\right)$, one may consider the subset $\Sigma(\xi) \subset \tilde{S}$ of those points $\tilde{t} \in \tilde{S}$ for which $\xi(\tilde{t})$ is a Hodge class. By construction, $\xi(\tilde{t})$ is an element of the rational vector space $W \times\{\tilde{t}\}$, so it is a Hodge class if and only if its image in the vector bundle $u^{*} \mathscr{W} \otimes_{\mathbb{Q}} \mathscr{O}_{\tilde{S}}$ lies in the holomorphic subbundle $\mathrm{Fil}^{0}$. In this way we see that $\Sigma(\xi)$ is a countable union of irreducible analytic subvarieties of $\tilde{S}$. If we start with a Hodge class $\xi(\tilde{s})$, it may happen that $\Sigma(\xi)=S$; this is equivalent to saying that at every point $\tilde{t} \in \tilde{S}$, the class $\xi(\tilde{t}) \in W \times\{\tilde{t}\}$ that is obtained from $\xi(\tilde{s})$ by horizontal transport is again a Hodge class. One obtains the exceptional locus $\operatorname{Exc}(\mathscr{V}) \subset S$ as the image in $S$ of the union of all loci $\Sigma(\xi)$ (for all 
tensor contructions $\mathscr{W}$ and all $\left.\xi \in \Gamma\left(\tilde{S}, u^{*} \bar{W}\right)\right)$ for which $\Sigma(\xi)$ is strictly contained in $\tilde{S}$. The fact that the components of the exceptional locus are in fact algebraic subvarieties of $S$ is a deep result of Cattani, Deligne and Kaplan [24].

We now turn to the relation with the algebraic monodromy group, which, as we will see, is one of the main reasons why working with families of varieties (or motives) is so effective.

Theorem 4.1.3. Let $S$ be an irreducible nonsingular complex algebraic variety with a base point $b \in S$. Let $\mathscr{V}$ be a polarizable $\mathbb{Q}$-VHS on $S$ that admits a $\mathbb{Z}$-structure. Let $V=\mathscr{V}_{b}$, let $G_{\text {mono }}(\overline{\mathscr{V}} / S) \subset \mathrm{GL}(V)$ be the algebraic monodromy group, and let $\operatorname{MT}(\mathscr{V} / S) \subset \mathrm{GL}(V)$ be the generic Mumford-Tate group of $\mathscr{V}$.

(i) The connected algebraic monodromy group $G_{\text {mono }}^{0}(\overline{\mathscr{V}} / S)$ is a semisimple normal subgroup of $\operatorname{MT}(\mathscr{V} / S)$.

(ii) If there is an $s \in S$ for which the Hodge structure $\mathscr{V}_{s}$ is of CM type then $G_{\text {mono }}^{0}(\overline{\mathscr{V}} / S)=\operatorname{MT}^{\mathrm{der}}(\mathscr{V} / S)$.

Part (i), except for the normality statement, is due to Deligne; see [31], Théorème 4.2.6 and [32], Proposition 7.5. The remaining assertions are due to André and can be found, in a more general form, in Sections 5-6 of [3]. In deriving this theorem, the Theorem of the Fixed Part plays an essential role. (See [71], Section 7 or, in a geometric setting, [31], Section 4.) We will come back to the latter in Theorem 4.3.1 below.

The automorphism group of the fibre functor $\mathrm{Fib}_{b}$ on the Tannakian subcategory $\langle\mathscr{V}\rangle \subset \operatorname{VHS}^{\text {pol }}(S)_{\mathbb{Q}}$ is the algebraic subgroup of $\mathrm{GL}(V)$ generated by the (fibre at $b$ of $)$ the generic Mumford-Tate group $\operatorname{MT}(\mathscr{V} / S) \subset \mathrm{GL}(V)$ of $\mathscr{V}$ together with the algebraic monodromy group $G_{\text {mono }}(\overline{\mathscr{V}} / S) \subset \mathrm{GL}(V)$ (which normalizes $\operatorname{MT}(\mathscr{V} / S)$ ). In particular, if $G_{\text {mono }}(\overline{\mathscr{V}} / S)$ is connected then this group is just $\operatorname{MT}(\mathscr{V} / S)$ and we obtain an equivalence of tensor categories between $\langle\mathscr{V}\rangle$ and $\operatorname{Rep}(\operatorname{MT}(\mathscr{V} / S))$.

The proof of part (ii) of the theorem is not hard to understand and closer inspection of the argument in fact gives something a little stronger. Namely, if $Z$ is the centre of $\operatorname{MT}(\mathscr{V} / S)$ and $M_{1}, \ldots, M_{r}$ are its $\mathbb{Q}$-simple factors, ordered in such a way that we have $G_{\text {mono }}(\mathscr{V} / S)=M_{t+1} \cdot M_{t+2} \cdots M_{r}$ for some $t \geq 0$, then for every $s \in S$ the Mumford-Tate group of $\mathscr{V}_{s}$ contains a normal subgroup isogenous to $Z \cdot M_{1} \cdots M_{t}$. See also the first assertion in Theorem 4.3 .8 below.

\subsection{Families of Galois representations}

We now discuss some $\ell$-adic analogues of the above results about variations of Hodge structure. Consider a nonsingular and geometrically connected variety $S$ over a field $K$ of characteristic 0 . Fix an algebraic closure $K \subset \bar{K}$ and a geometric base point $b$ of $S_{\bar{K}}$. We use the same notation $b$ for the induced base points of $S$ and of $\operatorname{Spec}(K)$. In what follows, $\pi_{1}(S, b)$ denotes the Grothendieck fundamental group.

In their barest form, the families of Galois representations that we will consider are just the lisse $\mathbb{Q}_{\ell}$-sheaves on $S$ that admit an integral structure. Such a sheaf $\mathscr{V}_{\ell}$ is given by a continuous representation

$$
\rho_{\ell}: \pi_{1}(S, b) \rightarrow \mathrm{GL}\left(V_{\ell}\right)
$$


where $V_{\ell}=\mathscr{V}_{\ell, b}=b^{*}\left(\mathscr{V}_{\ell}\right)$ is the fibre of $\mathscr{V}_{\ell}$ at $b$. Note that by compactness of the fundamental group, $V_{\ell}$ contains a $\mathbb{Z}_{\ell}$-lattice that is stable under the action of $\pi_{1}(S, b)$. We define $G_{\ell}\left(\mathscr{V}_{\ell} / S\right) \subset \mathrm{GL}\left(V_{\ell}\right)$ to be the Zariski closure of the image of $\rho_{\ell}$. For $S=\operatorname{Spec}(K)$ we recover the groups $G_{\ell}(H)$ that we have studied in Section 2.2.

We denote by $\overline{\mathscr{V}}_{\ell}$ the restriction of $\mathscr{V}_{\ell}$ to $S_{\bar{K}}$. It is sometimes useful to view $G_{\ell}\left(\mathscr{V}_{\ell} / S\right)$ as an $\ell$-adic local system of algebraic subgroups of $\operatorname{GL}\left(\overline{\mathscr{V}_{\ell}}\right)$, whose fibre at any geometric point $\bar{s}$ is the image of $\pi_{1}(S, \bar{s})$ in its monodromy representation on $\mathscr{V}_{\ell, \bar{s}}$. (Remark 4.1.1 has an obvious $\ell$-adic analogue.) For reasons to become clear (see Remark 4.2.3), we refer to $G_{\ell}\left(\mathscr{V}_{\ell} / S\right)$ as the generic $\ell$-adic algebraic Galois group of $\mathscr{V}_{\ell}$ over $S$.

Galois-generic points. Recall that we have a short exact sequence

$$
1 \longrightarrow \pi_{1}\left(S_{\bar{K}}, b\right) \longrightarrow \pi_{1}(S, b) \longrightarrow \Gamma_{K} \longrightarrow 1
$$

with $\Gamma_{K}=\operatorname{Gal}(\bar{K} / K)=\pi_{1}(\operatorname{Spec}(K), b)$. We define $G_{\text {mono }}\left(\overline{\mathscr{V}}_{\ell} / S_{\bar{K}}\right) \subset \operatorname{GL}\left(V_{\ell}\right)$ to be the Zariski closure of $\rho_{\ell}\left(\pi_{1}\left(S_{\bar{K}}, b\right)\right)$. By construction it is a normal algebraic subgroup of $G_{\ell}\left(\mathscr{V}_{\ell} / S\right)$. Note that this monodromy group only depends on $\overline{\mathscr{V}}_{\ell} / S_{\bar{K}}$, so the notation is justified. It is sometimes more convenient for us to work with the corresponding local systems on $S_{\bar{K}}$ of algebraic groups

$$
G_{\text {mono }}\left(\overline{\mathscr{V}_{\ell}} / S_{\bar{K}}\right) \triangleleft G_{\ell}\left(\mathscr{V}_{\ell} / S\right) \subset \operatorname{GL}\left(\overline{\mathscr{V}_{\ell}}\right) .
$$

For a point $s \in S$ with residue field $\kappa(s)$ we can complete (4.1) to a commutative diagram

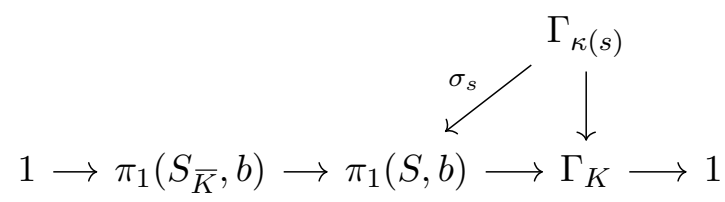

in which $\sigma_{s}$ is independent of choices only up to conjugacy by an element of $\pi_{1}\left(S_{\bar{K}}, b\right)$. For the discussion that follows, it is important to note that $\Gamma_{\kappa(s)} \rightarrow \Gamma_{K}$ has open image.

Definition 4.2.1. A point $s \in S$ is said to be Galois-generic (with respect to the lisse $\mathbb{Q}_{\ell}$-sheaf $\left.\mathscr{V}_{\ell}\right)$ if the image of $\rho_{\ell} \circ \sigma_{s}$ is open in the image of $\rho_{\ell}$. A point $s \in S(\bar{K})$ is said to be Galois-generic (with respect to $\mathscr{V}_{\ell}$ ) if its image point in $S$ is Galois-generic in the previous sense.

Note that we here consider the actual images of $\rho_{\ell}$ and $\rho_{\ell} \circ \sigma_{s}$, and not their Zariski closures. See however Remark 4.2.3. We write $\operatorname{Ggen}\left(\mathscr{V}_{\ell}\right)$ or $\operatorname{Ggen}\left(\mathscr{V}_{\ell} / S\right)$ for the set of Galois-generic points and $\operatorname{Exc}\left(\mathscr{V}_{\ell}\right) \subset S$ for its complement.

Some useful basic properties concerning this notion can be found in Section 3.2 of [20]. Apart from the fact that the generic point $\eta$ of $S$ is Galois-generic, it is not clear a priori if there are any other Galois-generic points. One can say more if the field $K$ is hilbertian, which is the case, for instance, if $K$ is finitely generated over $\mathbb{Q}$. (See [85], Section 9.5.) In that case, it follows from the results in [85], Section 10.6, that for $d$ large enough there are infinitely many Galois-generic points $s \in S$ with 
$[\kappa(s): K] \leq d$. If additionally $S$ is a curve, we have the following much more precise result of Cadoret and Tamagawa. As explained in [22], Section 5.2, the technical condition that $\mathfrak{g}^{\mathrm{ab}}=0$ that appears in the statement is always satisfied in the examples that are of interest to us.

Theorem 4.2.2 (Cadoret-Tamagawa). Let $S$ be a geometrically connected nonsingular curve over a field $K$ that is finitely generated over $\mathbb{Q}$. As above, let $\mathscr{V}_{\ell}$ be a lisse $\mathbb{Q}_{\ell}$ sheaf on $S$ that admits a $\mathbb{Z}_{\ell}$-structure. Let $\mathfrak{g}$ be the Lie algebra of $\rho_{\ell}\left(\pi_{1}\left(S_{\bar{K}}, b\right)\right)$, and assume that $\mathfrak{g}^{\mathrm{ab}}=0$. Then for every $d \geq 1$ the set

$$
\operatorname{Exc}\left(\mathscr{V}_{\ell}\right)^{\leq d}=\left\{s \in \operatorname{Exc}\left(\mathscr{V}_{\ell}\right) \mid[\kappa(s): K] \leq d\right\}
$$

is finite, and there is an integer $B_{d}\left(\mathscr{V}_{\ell}\right)$ such that for all $s \in \operatorname{Ggen}\left(\mathscr{V}_{\ell} / S\right)$ with $[\kappa(s): K] \leq d$ we have

$$
\left[\operatorname{Im}\left(\rho_{\ell}\right): \operatorname{Im}\left(\rho_{\ell} \circ \sigma_{s}\right)\right] \leq B_{d}\left(\mathscr{V}_{\ell}\right)
$$

This result is Theorem 1.1 in [23]. It is an open problem how to extend such a result to lisse $\mathbb{Q}_{\ell}$-sheaves over a base variety of higher dimension. (It is not even clear what is the expected statement.)

Remark 4.2.3. The lisse $\ell$-adic sheaves in which we are interested are those coming from families of motives; see the next section. These have the property that for every point $s \in S$ the image of $\rho_{\ell} \circ \sigma_{s}$ is open in the group of $\mathbb{Q}_{\ell}$-points of its Zariski closure. To see this one needs the following. If $K$ is a number field then the $\ell$-adic Galois representation associated with a motive over $K$ has the property that it is a Hodge-Tate representation at all places above $\ell$; this follows from a result of Faltings (see [46], Corollaire 2.1.3) together with the fact that any subquotient of a Hodge-Tate representation is again Hodge-Tate. By a result of Bogomolov [14], this property implies that the image of such a representation is open in the $\mathbb{Q}_{\ell^{-}}$ points of its Zariski closure. This extends to fields $K$ that are finitely generated over $\mathbb{Q}$ by using Hilbert's irreducibility theorem, as discussed above; see again [85], Section 10.6, or [81].

For such $\ell$-adic sheaves it follows that $s \in S$ is Galois-generic if and only if the inclusion $G_{\ell}^{0}\left(\mathscr{V}_{\ell, s}\right) \subset G_{\ell}^{0}\left(\mathscr{V}_{\ell} / S\right)$ is an equality. This property, together with the abundance of Galois-generic points (assuming the base variety $S$ to be defined over a finitely generated field $K)$ justifies calling $G_{\ell}^{0}\left(\mathscr{V}_{\ell} / S\right)$ the generic $\ell$-adic Galois group of $\mathscr{V}_{\ell} / S$.

\subsection{Families of motives}

Again let $S$ be a nonsingular geometrically connected variety over a field $K$ of characteristic 0 . Let $f: X \rightarrow S$ be a projective smooth morphism. Fixing $i \geq 0$ and $n \in \mathbb{Z}$, consider the lisse $\mathbb{Q}_{\ell}$-sheaf $\mathscr{H}_{\ell}=R^{i} f_{*}\left(\mathbb{Q}_{\ell}(n)\right)$. If $K=\mathbb{C}$ we may also consider $\mathscr{H}=R^{i} f_{*}(\mathbb{Q}(n))$, which is a variation of Hodge structure on $S^{\text {an }}$. Let $\xi \in \mathrm{H}^{0}\left(S_{\bar{K}}, \mathscr{H}_{\ell}\right)$ (respectively $\xi \in \mathrm{H}^{0}(S, \mathscr{H})$ ) be a global section. If $b \in S(\bar{K})$ is a geometric base point, $\xi$ may also be given as a $\pi_{1}\left(S_{\bar{K}}, b\right)$-invariant class in the fibre $\mathrm{H}_{\ell}=\mathscr{H}_{\ell, b}=\mathrm{H}^{i}\left(X_{b}, \mathbb{Q}_{\ell}\right)$ (resp. $\mathrm{H}=\mathscr{H}_{b}=\mathrm{H}^{i}\left(X_{b}, \mathbb{Q}\right)$ ). If $\mathrm{P}$ is a property of 
such cohomology classes, one may wonder if this property is stable under parallel transport. In other words: if $\xi(s)$ has property $\mathrm{P}$ for some point $s$, does it follow that all $\xi(t)$ have this property $\mathrm{P}$ ?

Theorem 4.3.1. Notation and assumptions as above.

(i) With $K=\mathbb{C}$, if $\xi(s) \in \mathrm{H}^{i}\left(X_{s}, \mathbb{Q}(n)\right)$ is a Hodge class for some $s \in S(\mathbb{C})$ then $\xi(t)$ is a Hodge class for every $t \in S(\mathbb{C})$.

(ii) If $\xi(s) \in \mathrm{H}^{i}\left(X_{s}, \mathbb{Q}_{\ell}(n)\right)$ is a Tate class for some closed point $s \in S$ then $\xi(t)$ is a Tate class for every closed point $t$.

(iii) If $\xi(s) \in \mathrm{H}^{i}\left(X_{s}, \mathbb{Q}_{\ell}(n)\right)$ is a motivated cycle for some point $s \in S$ then $\xi(t)$ is a motivated cycle for every point $t$.

Part (i) follows from, and is essentially equivalent to, the Theorem of the Fixed Part; see [71], Corollary 7.23. (Of course, the existence of nonzero Hodge classes implies that $i=2 n$.) Part (ii) is essentially trivial: since by assumption $\xi(s) \in$ $\mathrm{H}^{i}\left(X_{s}, \mathbb{Q}_{\ell}(n)\right)$ is invariant under the action of $\pi_{1}\left(S_{\bar{K}}, b\right)$, if $\xi(s)$ is a Tate class then it is invariant under an open subgroup of $\pi_{1}(S, b)$. This implies that the same is true at every closed point $t$, hence $\xi(t)$ is again a Tate class. Part (iii), which is much deeper, is one of the main results (Théorème 0.5) of André's article [5].

Remark 4.3.2. Take $K=\mathbb{C}$. If for "P" we take the stronger property of being the cohomology class of an algebraic cycle, we arrive at the Variational Hodge Conjecture (VHC) as formulated by Grothendieck in [40], footnote 13 on page 103 . With $\xi \in$ $\mathrm{H}^{0}\left(S, R^{i} f_{*} \mathbb{Q}(n)\right)$ as above, this is the assertion that if $\xi(s) \in \mathrm{H}^{i}\left(X_{s}, \mathbb{Q}(n)\right)$ is an algebraic cycle class for some $s \in S(\mathbb{C})$, the same is true for all $\xi(t)$. It follows from Theorem 4.3.1(i) that the Hodge conjecture implies the Variational Hodge Conjecture, and the latter should in fact be viewed as a key (conjectural) step towards the Hodge conjecture. It is known that the VHC for abelian schemes implies the Hodge conjecture for abelian varieties; see [1], Section 6 or [5], Section 6.

Combining Theorem 4.3.1(iii) with Remark 3.1.5, we see that the Standard Conjecture B implies the VHC. See [7] for a partial extension of this to families in positive characteristic.

Definition 4.3.3. Let $S$ be a nonsingular geometrically connected variety over a field $K$ of characteristic 0 . By a family of motives over $S$ we mean a triple $\mathbf{M}=$ $(X, e, n)$ with

- $f: X \rightarrow S$ a projective smooth morphism with connected fibres,

- $e$ a global section of $R^{2 d}(f \times f)_{*} \mathbb{Q}_{\ell, X \times{ }_{S} X}(d)$, where $d$ is the relative dimension of $X / S$,

- $n$ an integer,

such that for every $s \in S$ (or, equivalently, some $s \in S$ ) the value $e(s) \in \mathrm{H}^{2 d}\left(X_{s} \times_{\kappa(s)}\right.$ $\left.X_{s}, \mathbb{Q}_{\ell}(d)\right)$ is a projector in $\operatorname{Corr}_{\text {mot }}^{0}\left(X_{s}, X_{s}\right)=A_{\text {mot }}^{d}\left(X_{s} \times_{\kappa(s)} X_{s}\right)$, as in Section 3.1.

Let us note that, a priori, such families are more general than those considered in [5], Section 5.2. With $\mathbf{M}$ as in the definition, the fibre $\mathbf{M}_{s}=\left(X_{s}, e_{s}, n\right)$ at a point 
$s \in S$ is a motive over $\kappa(s)$. If the base field is $\mathbb{C}$ we may also realize the projector $e$ as a global section of $R^{2 d}(f \times f)_{*} \mathbb{Q}_{X \times{ }_{S} X}(d)$.

Realizations. Assume the base field $K$ is finitely generated over $\mathbb{Q}$. Let $\mathbf{M}=(X, e, n)$ be a family of motives over $S$. As in Section 3.2 we can consider the Hodge realizations and $\ell$-adic realizations of this family. Given a complex embedding $\sigma: K \rightarrow \mathbb{C}$, we define $\mathscr{H}_{\sigma}(\mathbf{M})$ to be the variation of Hodge structure on $S_{\sigma}^{\text {an }}=\left(S \otimes_{K, \sigma} \mathbb{C}\right)^{\text {an }}$ that is obtained as

$$
\mathscr{H}_{\sigma}(\mathbf{M})=e \cdot\left(\bigoplus_{i \geq 0} R^{i} f_{\sigma, *}^{\text {an }} \mathbb{Q}\right)(n)
$$

where $f_{\sigma}: X_{\sigma} \rightarrow S_{\sigma}$ is the morphism obtained from $f$ by base change. We denote the underlying local system by $\overline{\mathscr{H}_{\sigma}}(\mathbf{M})$. Similarly, for $\ell$ a prime number, let

$$
\mathscr{H}_{\ell}(\mathbf{M})=e \cdot\left(\bigoplus_{i \geq 0} R^{i} f_{*} \mathbb{Q}_{\ell}\right)(n)
$$

which is a lisse $\mathbb{Q}_{\ell}$-sheaf on $S$. We denote by $\overline{\mathscr{H}}_{\ell}(\mathbf{M})$ its restriction to $S_{\bar{K}}$. To understand the role of this local system, note that in working with a single motive over a field $K$, we have used the symbol $\mathrm{H}_{\ell}(\mathbf{M})$ for both the associated Galois representation and its underlying vector space. One should think of the Galois representation as an $\ell$-adic sheaf on $\operatorname{Spec}(K)$; the underlying vector space is then the pull-back of this sheaf to $\operatorname{Spec}(\bar{K})$. In that situation, using the same notation $\mathrm{H}_{\ell}(\mathbf{M})$ is not likely to cause any confusion. When working over a base variety $S$, however, the underlying vector space gets replaced by a local system on $S_{\bar{K}}$, and it seems a good idea to use a special notation for this object, namely $\overline{\mathscr{H}}_{\ell}(\mathbf{M})$.

The local systems $\overline{\mathscr{H}}_{\sigma}(\mathbf{M})$ on $S_{\sigma}^{\text {an }}$ and $\overline{\mathscr{H}}_{\ell}(\mathbf{M})$ on $S_{\bar{K}}$ may be compared once we choose an embedding $\tilde{\sigma}: \bar{K} \rightarrow \mathbb{C}$ with $\left.\tilde{\sigma}\right|_{K}=\sigma$. To express this, take a base point $b \in S(\bar{K})$. We again write $b$ for the induced $\mathbb{C}$-valued point of $S_{\sigma}$. The morphism $S_{\sigma} \rightarrow S_{\bar{K}}$ induces an isomorphism $\pi_{1}\left(S_{\bar{K}}, b\right) \cong \pi_{1}\left(S_{\sigma}, b\right)$, and the latter group is the pro-finite completion of $\pi_{1}\left(S_{\sigma}^{\text {an }}, b\right)$. Writing $\mathrm{H}_{\sigma}=\mathscr{H}_{\sigma}(\mathbf{M})_{b}$ and $\mathrm{H}_{\ell}=\overline{\mathscr{H}}_{\ell}(\mathbf{M})_{b}$, we have a comparison isomorphism $I_{\tilde{\sigma}, \ell}: \mathrm{H}_{\sigma} \otimes \mathbb{Q}_{\ell} \sim \mathrm{H}_{\ell}$. The comparison of local systems then takes the form of a commutative diagram

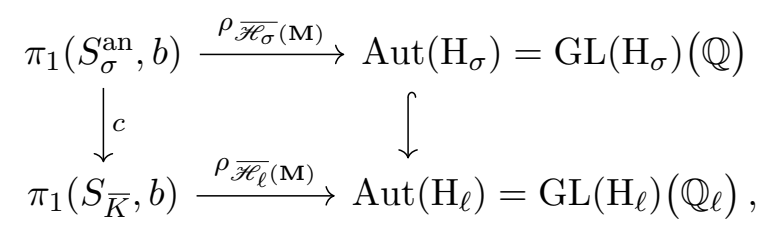

in which $c$ is the natural map from $\pi_{1}\left(S_{\sigma}^{\mathrm{an}}, b\right)$ to its pro-finite completion, which is $\pi_{1}\left(S_{\sigma}, b\right)$, followed by the isomorphism $\pi_{1}\left(S_{\sigma}, b\right) \stackrel{\sim}{\longrightarrow} \pi_{1}\left(S_{\bar{K}}, b\right)$. The following result is well-known but as it is important for the discussion, let us make it explicit.

Lemma 4.3.4. The isomorphism $\mathrm{GL}\left(\mathrm{H}_{\sigma}\right) \otimes \mathbb{Q}_{\ell} \sim \mathrm{GL}\left(\mathrm{H}_{\ell}\right)$ that is induced by $I_{\tilde{\sigma}, \ell}$ restricts to an isomorphism

$$
G_{\text {mono }}\left(\overline{\mathscr{H}_{\sigma}}(\mathbf{M}) / S_{\sigma}\right) \otimes \mathbb{Q}_{\ell} \sim G_{\text {mono }}\left(\overline{\mathscr{H}}_{\ell}(\mathbf{M}) / S_{\bar{K}}\right)
$$


Proof. The image of $c$ is dense in $\pi_{1}\left(S_{\bar{K}}, b\right)$, so $G_{\text {mono }}\left(\overline{\mathscr{H}}_{\ell}(\mathbf{M}) / S_{\bar{K}}\right)$ is the Zariski closure of the image of $\pi_{1}\left(S_{\sigma}^{\mathrm{an}}, b\right)$ in $\mathrm{GL}\left(\mathrm{H}_{\ell}\right)$. As this image is contained in $\mathrm{GL}\left(\mathrm{H}_{\sigma}\right)(\mathbb{Q})$, the assertion follows.

Remark 4.3.5. The above gives a "pointwise" comparison of local systems: we compare fibres of the local systems $\overline{\mathscr{H}}_{\sigma}(\mathbf{M})$ and $\overline{\mathscr{H}}_{\ell}(\mathbf{M})$ as representations of the fundamental group. For what follows it is more natural to directly compare $\overline{\mathscr{H}_{\sigma}}(\mathbf{M}) \otimes \mathbb{Q}_{\ell}$ and $\overline{\mathscr{H}}_{\ell}(\mathbf{M})$. This of course requires some care, as the first is a local system on $S_{\sigma}^{\text {an }}$ whereas the second is an $\ell$-adic local system on $S_{\bar{K}}$. To compare the two, one uses that there is a morphism of topoi $\left(\varepsilon^{*}, \varepsilon_{*}\right): S_{\sigma}^{\text {an }} \rightarrow S_{\sigma, \text { ét }}$ (compare [11], Section 6.1); the assertion is then that $\overline{\mathscr{H}_{\sigma}}(\mathbf{M}) \otimes \mathbb{Q}_{\ell} \cong \varepsilon^{*}\left(\sigma^{*}\left(\mathscr{H}_{\ell}(\mathbf{M})\right)\right)$, where $\sigma^{*}\left(\mathscr{H}_{\ell}(\mathbf{M})\right)=$ $\tilde{\sigma}^{*}\left(\overline{\mathscr{H}}_{\ell}(\mathbf{M})\right)$ is the pull-back of $\mathscr{H}_{\ell}(\mathbf{M})$ to an $\ell$-adic local system on $S_{\sigma}=\left(S_{\bar{K}}\right)_{\tilde{\sigma}}$.

The generic motivic Galois group. The following result of André (see [5], Théorème 5.2) gives an analogue of Theorem 4.1.2 for motivic Galois groups. We again consider a family of motives $\mathbf{M} / S$ with $S$ a geometrically connected nonsingular variety over a field $K$ that is finitely generated over $\mathbb{Q}$. If $\sigma$ is a complex embedding of $K$ we write $\operatorname{MT}\left(\mathbf{M}_{\sigma} / S_{\sigma}\right)$ for the generic Mumford-Tate group of the VHS $\mathscr{H}_{\sigma}(\mathbf{M})$ on $S_{\sigma}^{\text {an }}$, and we view it as a locally constant subgroup

$$
\operatorname{MT}\left(\mathbf{M}_{\sigma} / S_{\sigma}\right) \subset \operatorname{GL}\left(\overline{\mathscr{H}_{\sigma}}(\mathbf{M})\right) .
$$

If $\ell$ is a prime number, we write $G_{\ell}(\mathbf{M} / S)$ for the generic $\ell$-adic algebraic Galois group $G_{\ell}\left(\mathscr{H}_{\ell}(\mathbf{M}) / S\right)$ of the lisse $\ell$-adic sheaf $\mathscr{H}_{\ell}(\mathbf{M})$ on $S$, and we view it as a locally constant subgroup

$$
G_{\ell}(\mathbf{M} / S) \subset \operatorname{GL}\left(\overline{\mathscr{H}_{\ell}}(\mathbf{M})\right)
$$

Theorem 4.3.6 (André). Notation and assumptions as above. There exists a countable collection of reduced closed subschemes $\mathscr{E}_{i} \subsetneq S$ such that the following are true.

(i) For $\sigma: K \rightarrow \mathbb{C}$ a complex embedding, there is a locally constant subgroup scheme $\mathscr{G}_{\sigma}=G_{\mathrm{mot}, \sigma}(\mathbf{M} / S) \subset \mathrm{GL}\left(\overline{\mathscr{H}}_{\sigma}(\mathbf{M})\right)$ on $S_{\sigma}^{\text {an }}$ such that

(a) for every $s \in S_{\sigma}(\mathbb{C})$ we have $G_{\operatorname{mot}, \mathrm{B}}\left(\mathbf{M}_{s}\right) \subset \mathscr{G}_{\sigma, s}$;

(b) the inclusion $G_{\text {mot,B }}\left(\mathbf{M}_{s}\right) \subset \mathscr{G}_{\sigma, s}$ is strict if and only if $s \in \mathscr{E}_{i}(\mathbb{C})$ for some $i$.

(ii) For $\ell$ a prime number, there is a locally constant subgroup scheme $\mathscr{G}_{\ell}=$ $G_{\text {mot }, \ell}(\mathbf{M} / S) \subset \mathrm{GL}\left(\overline{\mathscr{H}}_{\ell}(\mathbf{M})\right)$ on $S_{\bar{K}}$ such that

(a) for every $s \in S(\bar{K})$ we have $G_{\operatorname{mot}, \ell}\left(\mathbf{M}_{s}\right) \subset \mathscr{G}_{\ell, s}$;

(b) the inclusion $G_{\text {mot, } \ell}\left(\mathbf{M}_{s}\right) \subset \mathscr{G}_{\ell, s}$ is strict if and only if $s \in \mathscr{E}_{i}(\bar{K})$ for some $i$.

See Remark 4.3.12 for some comments about the proof. In (i)(a), recall from Section 3.2 that $G_{\text {mot,B }}$ denotes the motivic Galois group of a motive over $\mathbb{C}$ with respect to the Betti fibre functor.

Remarks 4.3.7. (i) We denote the union of the subschemes $\mathscr{E}_{i}$ by $\operatorname{Exc}(\mathbf{M} / S)$ and we define the motivic generic locus $\operatorname{Mgen}(\mathbf{M} / S)$ to be the complement. Taking the union of the $\mathscr{E}_{i}$ has to be interpreted on the level of $\mathbb{C}$-valued or $\bar{K}$-valued points; e.g., $s \in S(\mathbb{C})$ is said to be a motivic generic point for $\mathbf{M} / S$ if $s \notin \cup_{i} \mathscr{E}_{i}(\mathbb{C})$. 
(ii) We call $G_{\text {mot }, \sigma}(\mathbf{M} / S)$ (Betti incarnation) or $G_{\text {mot }, \ell}(\mathbf{M} / S)$ ( $\ell$-adic incarnation) the generic motivic Galois group of $\mathbf{M} / S$. These two are "the same": with notation as in the discussion preceding Lemma 4.3.4, the isomorphism $\mathrm{GL}\left(\mathrm{H}_{\sigma}\right) \otimes \mathbb{Q}_{\ell} \stackrel{\sim}{\longrightarrow}$ $\mathrm{GL}\left(\mathrm{H}_{\ell}\right)$ on fibres at a point $b$ restricts to an isomorphism $G_{\operatorname{mot}, \sigma}(\mathbf{M} / S)_{b} \otimes \mathbb{Q}_{\ell} \stackrel{\sim}{\longrightarrow}$ $G_{\operatorname{mot}, \ell}(\mathbf{M} / S)_{b}$. We can also formulate the comparison in a more global way: with notation as in Remark 4.3.5, the local system of algebraic groups $G_{\operatorname{mot}, \sigma}(\mathbf{M} / S) \otimes \mathbb{Q}_{\ell}$ is isomorphic to $\varepsilon^{*}\left(G_{\operatorname{mot}, \ell}(\mathbf{M} / S)\right)$.

(iii) In our formulation of the theorem we take $\mathbf{M} / S$ as the primary object, whereas most assertions are about either the family of motives $\mathbf{M}_{\sigma}$ over $S_{\sigma}$ or the family $\mathbf{M}_{\bar{K}}$ over $S_{\bar{K}}$. It should be understood that the conclusions of the theorem apply to any family of motives $\mathbf{N} / T$ with $T$ a nonsingular variety over an algebraically closed field of characteristic 0 , as one can always find a model $\mathbf{M} / S$ of $\mathbf{N} / T$ over a finitely generated field $K$. Whether to view $\mathbf{N} / T$ as the principal object or $\mathbf{M} / S$ is a matter of choice. This may be compared to the two ways in which we have presented the Mumford-Tate conjecture, in 2.3.1 and 3.2.4: apart from the fact that in Section 2.3 we had not yet generalized the conjecture to motives, the two versions are different formulations of the same mathematical problem.

With $\mathbf{M} / S$ a family of motives as in the above discussion, the following result gives a nice connection between the various "generic loci" (Hodge, Galois, motivic) and the monodromy action. If we choose a complex embedding $\sigma: K \rightarrow \mathbb{C}$ we obtain a VHS $\mathscr{H}_{\sigma}(\mathbf{M})$ on $S_{\sigma}^{\text {an }}$ and, abbreviating $\overline{\mathscr{H}_{\sigma}}(\mathbf{M})$ to $\overline{\mathscr{H}_{\sigma}}$, we have local systems of algebraic groups

$$
G_{\text {mono }}^{0}\left(\overline{\mathscr{H}_{\sigma}} / S_{\sigma}\right) \subset \operatorname{MT}\left(\mathbf{M}_{\sigma} / S_{\sigma}\right) \subset G_{\text {mot }, \sigma}(\mathbf{M} / S) \subset \operatorname{GL}\left(\overline{\mathscr{H}_{\sigma}}\right) .
$$

For $s \in S(\mathbb{C})$ we have the Mumford-Tate group $\operatorname{MT}\left(\mathbf{M}_{s}\right) \subset \operatorname{MT}\left(\mathbf{M}_{\sigma} / S_{\sigma}\right)_{s}$ and the motivic Galois group $G_{\text {mot,B }}\left(\mathbf{M}_{s}\right) \subset G_{\text {mot }, \sigma}(\mathbf{M} / S)_{s}$ of the fibre $\mathbf{M}_{s}$. Similarly, we may choose a prime number $\ell$ and, abbreviating $\overline{\mathscr{H}}_{\ell}(\mathbf{M})$ to $\overline{\mathscr{H}}_{\ell}$, consider the local systems of algebraic groups on $S_{\bar{K}}$ :

$$
G_{\text {mono }}^{0}\left(\overline{\mathscr{H}}_{\ell} / S_{\bar{K}}\right) \subset G_{\ell}^{0}(\mathbf{M} / S) \subset G_{\text {mot }, \ell}(\mathbf{M} / S) \subset \mathrm{GL}\left(\overline{\mathscr{H}}_{\ell}\right) .
$$

For $s \in S(\bar{K})$ we have the $\ell$-adic algebraic Galois group $G_{\ell}^{0}\left(\mathbf{M}_{s}\right) \subset G_{\ell}^{0}(\mathbf{M} / S)_{s}$ and the motivic Galois group $G_{\text {mot }, \ell}\left(\mathbf{M}_{s}\right) \subset G_{\text {mot }, \ell}(\mathbf{M} / S)_{s}$ of $\mathbf{M}_{s}$.

Theorem 4.3.8. Notation and assumptions as above.

(i) For $\sigma$ a complex embedding of $K$, the connected algebraic monodromy group $G_{\text {mono }, \sigma}^{0}=G_{\text {mono }}^{0}\left(\overline{\mathscr{H}_{\sigma}} / S_{\sigma}\right)$ is a normal subgroup of the generic motivic Galois group $G_{\text {mot }, \sigma}(\mathbf{M} / S)$. For every $s \in S(\mathbb{C})$ we have

$$
G_{\mathrm{mono}, \sigma}^{0} \cdot \operatorname{MT}\left(\mathbf{M}_{s}\right)=\operatorname{MT}(\mathbf{M} / S)_{s}
$$

and

$$
G_{\mathrm{mono}, \sigma}^{0} \cdot G_{\mathrm{mot}, \sigma}\left(\mathbf{M}_{s}\right)=G_{\mathrm{mot}, \sigma}(\mathbf{M} / S)_{s} .
$$

(ii) Let $\ell$ be a prime number. Then the connected algebraic monodromy group $G_{\text {mono, } \ell}^{0}=G_{\text {mono }}^{0}\left(\overline{\mathscr{H}}_{\ell} / S\right)$ is a normal subgroup of the generic motivic Galois 
group $G_{\operatorname{mot}, \ell}(\mathbf{M} / S)$. For every $s \in S(\bar{K})$ we have

$$
G_{\mathrm{mono}, \ell}^{0} \cdot G_{\mathrm{mot}, \ell}\left(\mathbf{M}_{s}\right)=G_{\mathrm{mot}, \ell}(\mathbf{M} / S)_{s} .
$$

If moreover $G_{\ell}\left(\mathbf{M}_{s}\right)$ is reductive then

$$
G_{\mathrm{mono}, \ell}^{0} \cdot G_{\ell}\left(\mathbf{M}_{s}\right)=G_{\ell}(\mathbf{M} / S)_{s} .
$$

Proof. The normality of $G_{\text {mono }}^{0}$ in the generic motivic Galois group is part of [5], Théorème 0.6.4 (where it is stated at the level of Lie algebras). For $s \in S(\mathbb{C})$, let $Q=G_{\text {mono }}^{0} \cdot \operatorname{MT}\left(\mathbf{M}_{s}\right) \subset \mathrm{MT}(\mathbf{M} / S)_{s}$, and write $\mathrm{H}_{s}=\mathrm{H}_{\mathrm{B}}\left(\mathbf{M}_{s}\right)$ for the fibre of $\overline{\mathscr{H}_{\sigma}}$ at $s$. If $T_{s}=T\left(H_{s}\right)$ is any tensor space built from $H_{s}$, every $Q$-invariant tensor $\xi(s) \in T_{s}$ extends to a flat section $\xi$ of $T\left(\overline{\mathscr{H}_{\sigma}}\right)$. Moreover, since $\operatorname{MT}\left(\mathbf{M}_{s}\right) \subset Q$, the value of $\xi$ at $s$ is a Hodge class. Theorem 4.3.1(i) implies that $\xi(t)$ is a Hodge class for every $t \in S(\mathbb{C})$; hence $\xi(s)$ is invariant under $\operatorname{MT}(\mathbf{M} / S)_{s}$. As $Q \subset \operatorname{MT}(\mathbf{M} / S)_{s}$ is an inclusion of reductive groups and both have the same tensor invariants, it follows that $Q=\operatorname{MT}(\mathbf{M} / S)_{s}$. (Use [35], Proposition 3.1.(c) and cf. Remark 2.1.8.)

For the remaining assertions, the argument is the same, using parts (ii) and (iii) of Theorem 4.3.1. Note however, that for the version about $G_{\ell}\left(\mathbf{M}_{s}\right)$ we need to assume reductiveness for the argument to work.

Corollary 4.3.9. Notation and assumptions as above.

(i) For $s \in S(\mathbb{C})$ we have

$$
\begin{aligned}
s \text { is Hodge-generic } & \Longleftrightarrow G_{\text {mono }}^{0}\left(\overline{\mathscr{H}_{\sigma}} / S_{\sigma}\right)_{s} \subset \mathrm{MT}\left(\mathbf{M}_{s}\right) ; \\
s \text { is motivically generic } & \Longleftrightarrow G_{\text {mono }}^{0}\left(\overline{\mathscr{H}_{\sigma}} / S_{\sigma}\right)_{s} \subset G_{\text {mot }, \mathrm{B}}\left(\mathbf{M}_{s}\right) .
\end{aligned}
$$

(ii) For $s \in S(\bar{K})$ we have

$$
s \text { is Galois-generic } \Longrightarrow G_{\text {mono }}^{0}\left(\overline{\mathscr{H}}_{\ell} / S_{\bar{K}}\right)_{s} \subset G_{\ell}^{0}\left(\mathbf{M}_{s}\right),
$$

and if $G_{\ell}^{0}\left(\mathbf{M}_{s}\right)$ is reductive, the reverse implication is also true. Furthermore,

$$
s \text { is motivically generic } \Longleftrightarrow G_{\text {mono }}^{0}\left(\overline{\mathscr{H}}_{\ell} / S_{\bar{K}}\right)_{s} \subset G_{\text {mot }, \ell}\left(\mathbf{M}_{s}\right) \text {. }
$$

Proof. The direct implications follow from (4.2) and (4.3). For the reverse implications we may, passing to a finite cover of $S$, assume the algebraic monodromy group is connected; the assertions are then immediate from the previous theorem.

Corollary 4.3.10. We have

$$
\operatorname{Hgen}\left(\mathscr{H}_{\sigma}(\mathbf{M}) / S\right) \subset \operatorname{Mgen}(S(\mathbb{C})) \quad \text { and } \operatorname{Ggen}\left(\mathscr{H}_{\ell}(\mathbf{M}) / S\right) \subset \operatorname{Mgen}(S(\bar{K})) \text {. }
$$

Remark 4.3.11. We leave it to the reader to draw conclusions from Theorem 4.3.8 that are similar in spirit to Theorem 4.1.3(i). For instance, in 4.3.8(i), if there exists a point $s$ for which $G_{\operatorname{mot}, \sigma}\left(\mathbf{M}_{s}\right)$ is connected then the fibres of $G_{\operatorname{mot}, \sigma}(\mathbf{M} / S)$ are connected, and if $G_{\operatorname{mot}, \sigma}\left(\mathbf{M}_{s}\right)$ is a torus then $G_{\text {mono }, \sigma}^{0}=G_{\operatorname{mot}, \sigma}^{\text {der }}(\mathbf{M} / S)$.

Remark 4.3.12. Theorem 4.3.6 together with the last assertion of Corollary 4.3.9 is essentially the result proven by André in [5], Théorème 5.2. There are some minor differences, though. One of these is that André uses a more restricted notion of a 
family of motives. Let us sketch how one argues to deduce from André's theorem the results as we have stated them.

Over a dense open part $U \subset S$ we have a family of motives in the sense considered by André. We have the local systems of algebraic groups $G_{\text {mono }}\left(\overline{\mathscr{H}}_{\sigma}(\mathbf{M}) / S_{\sigma}\right)$ on $S_{\sigma}^{\text {an }}$. Its restriction to $U_{\sigma}$ is the algebraic monodromy group associated with the family of motives over $U$, and André's theorem gives us a local system of algebraic groups $\mathscr{G}_{U, \sigma}=G_{\text {mot, } \sigma}\left(\mathbf{M}_{U} / U\right)$. Because the monodromy groups on $U$ and $S$ are the same, this extends to a local system of algebraic groups $\mathscr{G}_{\sigma}=G_{\operatorname{mot}, \sigma}(\mathbf{M} / S) \subset$ $\operatorname{GL}\left(\overline{\mathscr{H}_{\sigma}}(\mathbf{M})\right)$ on $S_{\sigma}$. By using that the motivic Galois group can only go down under specialization (cf. [18], Sections $4-5$ ), we have $G_{\text {mot,B }}\left(\mathbf{M}_{s}\right) \subset \mathscr{G}_{\sigma, s}$ for every $s \in S_{\sigma}(\mathbb{C})$.

To define the motivic exceptional locus and show that it has the expected properties, let us fix an embedding $\tilde{\sigma}: \bar{K} \rightarrow \mathbb{C}$, as André does. We proceed by adding to $U$ a component $Z$ of the non-singular part of $S \backslash U$; this step will be iterated until we have reached $S$. We start by including in the exceptional locus on $U \cup Z$ the closure of the exceptional locus of $U$. Arguing by induction on the dimension, we may assume the theorem is true on $Z$. Now compare the local system of generic motivic Galois groups of $\mathbf{M}_{Z} / Z$ with the local system $\left.\mathscr{G}_{\sigma}\right|_{Z}$. If $G_{\text {mot, } \sigma}\left(\mathbf{M}_{Z} / Z\right)$ is strictly contained in $\left.\mathscr{G}_{\sigma}\right|_{Z}$, we add the entire $Z$ to the exceptional locus; else we only add $\operatorname{Exc}\left(\mathbf{M}_{Z} / Z\right)$. This construction gives that, for $s \in(U \cup Z)(\mathbb{C})$, the inclusion $G_{\text {mot,B }}\left(\mathbf{M}_{s}\right) \subset \mathscr{G}_{\sigma, s}$ is strict if and only if $s$ lies in the exceptional locus.

The next point is that we can also realize the generic motivic Galois group as a locally constant system $\mathscr{G}_{\ell}=G_{\text {mot }, \ell}(\mathbf{M} / S) \subset \mathrm{GL}\left(\overline{\mathscr{H}}_{\ell}(\mathbf{M})\right)$ on $S_{\bar{K}}$. This we do in two steps: (a) first go from $S_{\sigma}$ for the analytic topology to $S_{\sigma}$ with the étale topology; (b) next pass to $S_{\bar{K}}$ with the étale topology. Step (a) is essentially the same argument as in Lemma 4.3.4. For step (b) we use that the étale fundamental group does not change if we pass from $S_{\bar{K}}$ to $S_{\sigma}$. (As we are in characteristic 0, this follows from the Künneth formula for fundamental groups given in [39], Exposé XIII, Proposition 4.6.) Since we know that the "pointwise" motivic Galois groups are the same (see the isomorphism $\gamma_{\tilde{\sigma}, \ell}$ just before Conjecture 3.2.2), the properties stated in part (ii) of the Theorem 4.3.6 follow.

Finally, let us justify our claim that the exceptional locus is defined over $K$. For this, let $\alpha$ be an automorphism of $\bar{K} / K$, let $s \in S(\bar{K})$ and $t={ }^{\alpha} s$. We have to show that if $s$ is motivically generic, so is $t$. The group $G_{\operatorname{mot}, \ell}\left(\mathbf{M}_{t}\right)$ is an inner form of $G_{\text {mot }, \ell}\left(\mathbf{M}_{s}\right)$ : the latter is associated with the fibre functor on $\operatorname{Mot}(\bar{K})$ given by $\mathbf{N} \mapsto \mathrm{H}_{\ell}(\mathbf{N})$, whereas $G_{\text {mot }, \ell}\left(\mathbf{M}_{t}\right)$ is isomorphic to the motivic Galois group of $\mathbf{M}_{s}$ (sic!) associated with the fibre functor $\mathbf{N} \mapsto \mathrm{H}_{\ell}\left(\alpha^{*} \mathbf{N}\right)$. On the other hand, we have the inclusions $G_{\text {mot }, \ell}\left(\mathbf{M}_{t}\right) \subset \mathscr{G}_{\ell, t}$ and $G_{\text {mot }, \ell}\left(\mathbf{M}_{s}\right) \subset \mathscr{G}_{\ell, s}$, and the algebraic groups $\mathscr{G}_{\ell, t}$ and $\mathscr{G}_{\ell, s}$ are (non-canonically) isomorphic. Because $s$ is motivically generic, $G_{\text {mot, } \ell}\left(\mathbf{M}_{t}\right)$ has the same dimension and the same number of geometric components as $\mathscr{G}_{\ell, s}$; the same is then true for its inner twist $G_{\operatorname{mot}, \ell}\left(\mathbf{M}_{t}\right)$, and it follows that $t$ is motivically generic, too. 
Open problem 4.3.13. Prove that there are many Hodge-generic points over finite extensions of $K$. "Many" should mean here that over a 1-dimensional basis we have an analogue of the Cadoret-Tamagawa result, Theorem 4.2.2.

Applications to very general members in a family. One may try to deduce from the results that we have discussed conclusions about the motivic MTC for a very general member in a family, assuming we know it for some fibre.

Corollary 4.3.14. Let $\mathbf{M} / S$ be a family of motives, as before.

(i) Choose an embedding $\sigma: K \rightarrow \mathbb{C}$. Assume there is a point $t \in S(\mathbb{C})$ such that Conjecture $\mathrm{HM}_{\sigma}$ (see 3.2.2) for $\mathbf{M}_{t}$ is true. Then Conjecture $\mathrm{HM}_{\sigma}$ is true for $\mathbf{M}_{s}$ for all Hodge-generic points $s$.

(ii) Choose a prime number $\ell$. Assume there is a closed point $t \in S$ such that Conjecture $\mathrm{TM}_{\ell}$ (see 3.2.3) for $\mathbf{M}_{t}$ is true. Then Conjecture $\mathrm{TM}_{\ell}$ is true for $\mathbf{M}_{s}$ for all Galois-generic closed points s.

Proof. (i) Theorem 4.3.8, applied to the fibre $\mathbf{M}_{t}$, gives that $\operatorname{MT}(\mathbf{M} / S)=$ $G_{\text {mot }, \sigma}(\mathbf{M} / S)$. As $\operatorname{Hgen}\left(\mathscr{H}_{\sigma}(\mathbf{M}) / S\right) \subset \operatorname{Mgen}(S(\mathbb{C}))$, the assertion follows. For (ii) the argument is the same, where we note that if $G_{\ell}^{0}\left(\mathbf{M}_{t}\right)$ is reductive then so is $G_{\text {mono } \ell}^{0} \cdot G_{\ell}^{0}\left(\mathbf{M}_{t}\right)=G_{\ell}^{0}(\mathbf{M} / S)_{t}$.

We obtain a stronger conclusion if the fibres of $\mathbf{M} / S$ are abelian motives. Note that in this case the MTC is the same as the motivic MTC and is independent of $\sigma$ and $\ell$. (See Section 3.3.)

Corollary 4.3.15. In the situation of Corollary 4.3.14, assume the fibres $\mathbf{M}_{s}$ are abelian motives. If there is a closed point $t \in S$ such that the MTC is true for $\mathbf{M}_{t}$ then the MTC for $\mathbf{M}_{s}$ is true for all Galois-generic points s.

By Theorems 3.3.1 (in particular, its consequence (3.3)) and 3.3.2, the condition that the MTC is true for some fibre $\mathbf{M}_{t}$ is satisfied if there is a fibre with commutative Mumford-Tate group. (Such a fibre is usually called a CM fibre.)

\subsection{Some known results (3)}

Surfaces with $p_{g}=1$. It was proven by Tankeev [92], [93] and, independently, André [4] that the MTC is true for complex K3 surfaces. André's result works under some general assumptions; beyond K3 surfaces this gives the MTC for the $\mathrm{H}^{2}$ of a hyperkähler variety with second Betti number $>3$ and the $\mathrm{H}^{4}$ of a cubic fourfold. As already mentioned in Section 3.3, it is also proven that the corresponding motives lie in the category $\operatorname{Mot}^{\mathrm{Ab}}(\mathbb{C})$. The proofs use in an essential way the Kuga-Satake construction as formulated by Deligne in [32].

By a more elaborate argument that builds upon the ideas of Deligne and André, the author [55] was able to prove the MTC for arbitrary complex surfaces with $p_{g}=1$ under a mild condition on their moduli. (Recall that the geometric genus of a surface $X$ is $p_{g}(X)=\operatorname{dim}^{0}\left(X, \omega_{X}\right)=h^{2,0}(X)$.) The general form of the result is the following. 
Theorem 4.4.1. Let $Y$ be a non-singular complex projective variety of dimension d. Let $\mathbf{N} \subset \mathbf{H}^{2}(Y)$ be a submotive whose Hodge realization $\mathrm{H}_{\mathrm{B}}(\mathbf{N})$ has Hodge number $h^{2,0}=1$. Suppose there exists a connected nonsingular variety $S$, a smooth projective morphism $f: X \rightarrow S$ and a global section $e \in \Gamma\left(S, R^{2 d}(f \times f)_{*} \mathbb{Q}_{X \times X}(d)\right)$ such that

(a) $Y \cong X_{s}$ for some $s \in S(\mathbb{C})$;

(b) $e(s)$ is a projector that is of the form $e(s)=\pi_{2} \circ \operatorname{cl}(\gamma) \circ \pi_{2}$ for some $\gamma \in$ $\mathrm{CH}^{d}(X \times X) \otimes \mathbb{Q}$, where $\pi_{2}$ is the second Künneth projector on $Y$;

(c) via the isomorphism in (a), $\mathbf{N}$ is the submotive of $\mathbf{H}^{2}(Y)$ that is cut out by $e(s)$;

(d) if $\mathbf{M}=(X, e, 0)$ is the associated family of motives over $S$, the Variation of Hodge Structure $\mathscr{H}_{\mathrm{B}}(\mathbf{M})$ is not isotrivial.

Then the Tate conjecture and the Mumford-Tate conjecture for $\mathbf{N}$ are true.

The basic case of this result is of course when $\mathbf{N}=\mathbf{H}^{2}(Y)$ for a surface $Y$ with $p_{g}(Y)=1$; in that case the assumptions simply mean that $Y$ is a fibre in a family of surfaces for which the second cohomology groups give a non isotrivial VHS. Another case to which the theorem applies is when we have a surface $Y$ with $p_{g}(Y)=2$ and surjective albanese morphism $Y \rightarrow A$; in that case, $\mathbf{H}^{2}(Y)$ decomposes as the direct sum of $\mathbf{H}^{2}(A)$ and a submotive $\mathbf{N} \subset \mathbf{H}^{2}(Y)$ with Hodge number $h^{2,0}(\mathbf{N})=1$. In all such examples, in order to conclude that the TC and the MTC are true, one must find a family that satisfies the conditions of the theorem. A list of examples for which this has been carried out can be found in [55], Section 9.

Integral and adelic forms of the MTC. To simplify the exposition, let us here work over a subfield $K \subset \mathbb{C}$ that is of finite type, and define $\bar{K}$ to be the algebraic closure of $K$ in $\mathbb{C}$. (Phrased differently, the notation is the same as before but we fix an embedding $\tilde{\sigma}: \bar{K} \rightarrow \mathbb{C}$.) Also, let us restrict the discussion to motives of the form $\mathbf{M}=\mathbf{H}^{m}(Y)(n)$ for some nonsingular complete variety $Y$ over $K$, and let us assume throughout that $K=K^{\mathrm{conn}}$. (See 2.2.2(ii); as already remarked in 3.2 .1 , in the situation we consider $K^{\text {conn }}$ does not depend on $\ell$.) The integral cohomology modulo torsion gives us a lattice $\mathrm{H}_{\mathbb{Z}}$ inside $\mathrm{H}_{\mathrm{B}}=\mathrm{H}^{m}\left(Y_{\mathbb{C}}, \mathbb{Q}(n)\right)$. We may then view the Mumford-Tate group as a group scheme over $\mathbb{Z}$ by taking its integral closure inside $\mathrm{GL}\left(\mathrm{H}_{\mathbb{Z}}\right)$.

Let us assume that the MTC is true for the motive $\mathbf{H}^{m}(Y)(n)$. For every prime number $\ell$ we have a comparison isomorphism $\mathrm{H}_{\mathbb{Z}} \otimes \mathbb{Z}_{\ell} \stackrel{\sim}{\longrightarrow} \mathrm{H}_{\text {ét }}^{m}\left(Y_{\bar{K}}, \mathbb{Z}_{\ell}(n)\right)$ which we shall take as an identification. The associated $\ell$ adic Galois representation may then be viewed as a homomorphism

$$
\rho_{\ell}: \operatorname{Gal}(\bar{K} / K) \rightarrow \operatorname{MT}\left(\mathbb{Z}_{\ell}\right)
$$

and the image $\operatorname{Im}\left(\rho_{\ell}\right)$ is an open subgroup of $\mathrm{MT}\left(\mathbb{Z}_{\ell}\right)$ for the $\ell$ adic topology. (See Remark 4.2.3.) As the target group is compact, it follows that the index of $\operatorname{Im}\left(\rho_{\ell}\right)$ in $\operatorname{MT}\left(\mathbb{Z}_{\ell}\right)$ is finite.

We may also consider all prime numbers $\ell$ at the same time: writing $\hat{\mathrm{H}}=$ $\mathrm{H}_{\mathbb{Z}} \otimes \hat{\mathbb{Z}} \cong \prod_{\ell} \mathrm{H}_{\text {ét }}^{m}\left(Y_{\bar{K}}, \mathbb{Z}_{\ell}(n)\right)$, the product of the representations $\rho_{\ell}$ gives us a rep resentation

$$
\hat{\rho}: \operatorname{Gal}(\bar{K} / K) \rightarrow \operatorname{MT}(\hat{\mathbb{Z}}) .
$$


With this notation, the following two refinements of the MTC were proposed by Serre; see [79], Conjectures C.3.7 and C.3.8.

Conjecture 4.4.2. Notation and assumptions as above; in particular we assume the MTC is true for $\mathbf{H}^{m}(Y)(n)$.

(i) The index of $\operatorname{Im}\left(\rho_{\ell}\right)$ in $\mathrm{MT}\left(\mathbb{Z}_{\ell}\right)$ is bounded as $\ell$ varies. Moreover, for almost all $\ell$ the image $\operatorname{Im}\left(\rho_{\ell}\right)$ contains the commutator subgroup of $\mathrm{MT}\left(\mathbb{Z}_{\ell}\right)$, as well as all homotheties of the form $c^{m-2 n} \cdot \mathrm{id}$, for $c \in \mathbb{Z}_{\ell}^{\times}$.

(ii) If the Hodge realization $\mathrm{H}_{\mathrm{B}}$ is Hodge-maximal (see the next remark), the image of $\hat{\rho}$ is an open subgroup of $\mathrm{MT}(\hat{\mathbb{Z}})$.

By Hodge-maximality of $\mathrm{H}_{\mathrm{B}}$ we mean that there is no non-trivial isogeny of connected $\mathbb{Q}$-groups $M^{\prime} \rightarrow \mathrm{MT}_{\mathbb{Q}}$ such that the homomorphism $h: \mathbb{S} \rightarrow \mathrm{MT}_{\mathbb{R}}$ that defines the Hodge structure on $\mathrm{H}_{\mathrm{B}}$ lifts to a homomorphism $h^{\prime}: \mathbb{S} \rightarrow M_{\mathbb{R}}^{\prime}$. It is known (see [21], Remark 2.6) that Hodge-maximality is a necessary condition in order for $\operatorname{Im}(\hat{\rho})$ to be open in $\operatorname{MT}(\hat{\mathbb{Z}})$.

For elliptic curves, Serre himself proved in [78] that both parts of the conjecture are true. (In this case we know the MTC and $\mathrm{H}_{\mathrm{B}}$ is Hodge-maximal.) Much more recently, part (i) was proven for arbitrary abelian varieties (for which the usual MTC is true) by Hindry and Ratazzi [43] and, independently, Cadoret and the author [21]. In [21], also (ii) is proven for abelian varieties. For (the $\mathrm{H}^{2}$ of) $\mathrm{K} 3$ surfaces (for which, as discussed above, the MTC is true), it is shown in [21] that $\mathrm{H}_{\mathrm{B}}^{2}$ is Hodge-maximal and that the image of $\hat{\rho}$ is an open subgroup of $\operatorname{MT}(\hat{\mathbb{Z}})$.

Dependence of Ggen on $\ell$. If $\mathbf{M} / S$ is a family of motives then for every prime number $\ell$ we have, abbreviating $\mathscr{H}_{\ell}=\mathscr{H}_{\ell}(\mathbf{M})$, a (Galois-)exceptional locus $\operatorname{Exc}\left(\mathscr{H}_{\ell}\right)$ in $S$. Conjecture 3.2.3 predicts that these loci do not depend on $\ell$. If $\mathbf{M}$ is given by (the $\mathbf{H}^{1}$ of) an abelian scheme over $S$, Hui [45] proved that this is indeed true. It seems difficult to extend Hui's proof to more general cases, as it makes essential use of Faltings's theorem 2.2.7. To the author's knowledge, even for abelian motives the $\ell$-independence of $\operatorname{Exc}\left(\mathscr{H}_{\ell}\right)$ is not known in general. Cadoret [19] has shown that the $\operatorname{Exc}\left(\mathscr{H}_{\ell}\right)$ are independent of $\ell$ if the algebraic monodromy group of the family only has factors of Lie type A.

\subsection{An example}

We conclude with an application that illustrates how some of the techniques we have discussed can be used to prove results about algebraic cycles.

Theorem 4.5.1. For $d=5$ or $d=6$, let $X \subset \mathbb{P}_{\mathbb{C}}^{3}$ be a nonsingular surface given by an equation $s\left(T_{0}, T_{1}, T_{2}\right)+T_{3}^{d}=0$, with $s \in \mathbb{C}\left[T_{0}, T_{1}, T_{2}\right]_{d}$. Then the motive $\mathbf{H}(X)$ is an abelian motive and the Tate conjecture for $X$ is true.

We first prove this for $d=5$. Throughout the argument, let $E$ be the cyclotomic field $\mathbb{Q}[x] /\left(x^{4}+x^{3}+x^{2}+x+1\right)$ and write $\zeta_{5}=\exp (2 \pi i / 5)$. Let $\Sigma$ be the set of complex embeddings of $E$. In our calculations we identify it with $\mathbb{F}_{5}^{\times}$, letting $j \in \mathbb{F}_{5}^{\times}$ correspond to the embedding given by $x \mapsto \zeta_{5}^{j}$. 
Let $S \subset \mathbb{C}\left[T_{0}, T_{1}, T_{2}\right]_{5}$ be the affine subvariety of nonsingular homogeneous polynomials $s$ of degree 5 , and let $\pi: Y \rightarrow S$ be the smooth projective family of surfaces whose fibre at $s \in S$ is given by the equation $s\left(T_{0}, T_{1}, T_{2}\right)+T_{3}^{5}=0$. Let $\alpha$ be the automorphism of $Y / S$ given by $\left(y_{0}: y_{1}: y_{2}: y_{3}\right) \mapsto\left(y_{0}: y_{1}: y_{2}: \zeta_{5} \cdot y_{3}\right)$. We use the same notation $\alpha$ for the induced automorphisms of the fibres.

Via $\alpha$, the singular cohomology of each $Y_{s}$ gets the structure of an $E$-module in the category $\mathrm{HS}_{\mathbb{Q}}$. For $s \in S$ and $\sigma \in \Sigma$, write $\mathrm{H}^{p, q}\left(Y_{s}\right)(\sigma)$ for the space of $\xi \in \mathrm{H}^{p, q}\left(Y_{s}\right)$ such that $e(\xi)=\sigma(e) \cdot \xi$ for all $e \in E$. The dimensions $h^{p, q}(\sigma)$ of these spaces are independent of $s$ and may therefore be calculated on the Fermat surface of degree 5. Using [86], Theorem 1, we readily find:

$$
h^{2,0}(\overline{1})=3, \quad h^{2,0}(\overline{2})=1, \quad h^{2,0}(\overline{3})=0, \quad h^{2,0}(\overline{4})=0 .
$$

The subset $\Phi=\{\overline{1}, \overline{2}\} \subset \Sigma$ is a CM type. Consider the 2-dimensional abelian variety $B$ of $C M$ type $(E, \Phi)$; see Example 2.1.10. It is uniquely determined up to isogeny. Explicitly, $B$ can be realized as the Jacobian of the genus 2 curve given by $y^{2}=x^{5}-1$, with $E$-action induced by the automorphism $\beta$ given by $(x, y) \mapsto$ $\left(\zeta_{5} \cdot x, y\right)$. By construction,

$$
h^{1,0}(B)(\overline{1})=1, \quad h^{1,0}(B)(\overline{2})=1, \quad h^{1,0}(B)(\overline{3})=0, \quad h^{1,0}(B)(\overline{4})=0 .
$$

We use $B$ to construct "half twists" of the Hodge structures $\mathrm{H}^{2}\left(Y_{s}, \mathbb{Q}\right)$, as introduced by van Geemen in [100]. See also [101], Section 5. Our formulation of this construction is the one we introduced in [55], Section 7; see also [56], Section $3 .^{2}$ Namely, we consider the $\mathbb{Q}$-Hodge structures

$$
\underline{\operatorname{Hom}}_{E}\left(\mathrm{H}^{1}(B, \mathbb{Q}), \mathrm{H}^{2}\left(Y_{s}, \mathbb{Q}\right)\right)=\mathrm{H}^{1}(B, \mathbb{Q})^{\vee} \otimes_{E} \mathrm{H}^{2}\left(Y_{s}, \mathbb{Q}\right),
$$

which are sub-Hodge structures of $\underline{\operatorname{Hom}}\left(\mathrm{H}^{1}(B, \mathbb{Q}), \mathrm{H}^{2}\left(Y_{s}, \mathbb{Q}\right)\right)$. By our choice of $B$, these are polarizable $\mathbb{Q}$-Hodge structures of type $(1,0)+(0,1)$; hence they come from an abelian variety.

In fact, the construction can be done for the entire family over $S$. Denote by $\mathscr{H}^{2}(Y / S)$ the Variation of Hodge Structure over $S$ given by $R^{2} \pi_{*}\left(\mathbb{Q}_{Y}\right)$, and write $\mathrm{H}^{1}(B)_{S}$ for the constant VHS over $S$ with fibres $\mathrm{H}^{1}(B)=\mathrm{H}^{1}(B, \mathbb{Q})$. Both VHS have the structure of an $E$-module in the category $\vee \mathrm{HS}(S)_{\mathbb{Q}}$. Then

$$
\underline{\operatorname{Hom}}_{E}\left(\mathrm{H}^{1}(B)_{S}, \mathscr{H}^{2}(Y / S)\right)=\mathrm{H}^{1}(B)_{S}^{\vee} \otimes_{E} \mathscr{H}^{2}(Y / S)
$$

is a polarizable VHS over $S$ of type $(1,0)+(0,1)$. It admits an integral structure and also the structure of an $E$-module. Hence there exists an abelian scheme $a: A \rightarrow$ $S$ with endomorphisms by $E$ such that, writing $\mathscr{H}^{1}(A / S)$ for the VHS given by $R^{1} a_{*}\left(\mathbb{Q}_{A}\right)$,

$$
\mathscr{H}^{1}(A / S) \cong \underline{\operatorname{Hom}}_{E}\left(\mathrm{H}^{1}(B)_{S}, \mathscr{H}^{2}(Y / S)\right)
$$

It is important to note here that the abelian variety $B$ stays constant, and that $\mathrm{H}^{1}(B)$ is 1-dimensional as an $E$-vector space. By a Hom-tensor adjunction we find

\footnotetext{
${ }^{2}$ There is a misprint in the formula for $d(\sigma ;-1,0)$ that is given in [56], section 3.4. The third line in the displayed equation should say that $d(\sigma ;-1,0)=n$ if $\sigma \in \Phi \backslash\{\tau\}$.
} 
that there is an isomorphism

$$
u: \mathscr{H}^{1}(A / S) \otimes_{E} \mathrm{H}^{1}(B)_{S} \stackrel{\sim}{\longrightarrow} \mathscr{H}^{2}(Y / S) .
$$

We view the functor $-\otimes_{E} \mathrm{H}^{1}(B)$ as a "twisting" operation; note that it increases the weight by 1 and preserves the rank of the underlying local system.

Lemma 4.5.2. For every $s \in S(\mathbb{C})$ the fibre of the isomorphism $u$ at $s$ is the Betti realization of an isomorphism of motives

$$
\mathbf{u}_{s}:: \mathbf{H}^{1}\left(A_{s}\right) \otimes_{E} \mathbf{H}^{1}(B) \stackrel{\sim}{\longrightarrow} \mathbf{H}^{2}\left(Y_{s}\right) .
$$

Proof. Let $s_{0} \in S(\mathbb{C})$ be a point such that $Y_{s_{0}}$ is the Fermat surface. As the Fermat surface is an abelian motive (see the discussion in Section 3.3), the assertion is true for $s=s_{0}$. By Theorem 4.3.1(iii), it follows that $u_{s}$ is motivated for every $s$.

This proves that $\mathbf{H}^{2}\left(Y_{s}\right)$, and therefore also $\mathbf{H}\left(Y_{s}\right)=\mathbf{H}^{0}\left(Y_{s}\right) \oplus \mathbf{H}^{2}\left(Y_{s}\right) \oplus \mathbf{H}^{4}\left(Y_{s}\right)$, is an abelian motive. To deduce the Tate conjecture, we use the theorem of Faltings (Theorem 2.2.7, the case $\operatorname{char}(K)=0$ ). As $\mathbf{H}^{1}(B)(1) \cong \mathbf{H}^{1}(B)^{\vee}$ (via the choice of a polarization on $B$ ), we find that $\mathbf{H}^{2}\left(Y_{2}\right)(1)$ is isomorphic to a submotive of $\underline{\operatorname{Hom}}\left(\mathbf{H}^{1}(B), \mathbf{H}^{1}\left(A_{s}\right)\right)$, and as explained after Corollary 2.2.8, Faltings's result implies that every Tate class in the $\ell$-adic realization of the latter motive is algebraic. This concludes the proof of Theorem 4.5.1 for $d=5$.

For $d=6$ essentially the same argument works. We let $S \subset \mathbb{C}\left[T_{0}, T_{1}, T_{2}\right]_{6}$ be the affine subvariety of nonsingular homogeneous polynomials $s$ of degree 6 , and let $\pi: Y \rightarrow S$ be the smooth projective family of surfaces whose fibre at $s \in S$ is given by the equation $s\left(T_{0}, T_{1}, T_{2}\right)+T_{3}^{6}=0$. Let $\alpha$ be the automorphism of $Y / S$ given by $\left(y_{0}: y_{1}: y_{2}: y_{3}\right) \mapsto\left(y_{0}: y_{1}: y_{2}: \zeta_{6} \cdot y_{3}\right)$. The induced automorphism $\alpha^{*}$ makes the motive $\mathbf{H}^{2}\left(Y_{s}, \mathbb{Q}\right)$ a module over the group $\operatorname{ring} \mathbb{Q}[\mathbb{Z} / 6 \mathbb{Z}] \cong \mathbb{Q} \times \mathbb{Q} \times \mathbb{Q}\left(\zeta_{3}\right) \times \mathbb{Q}\left(\zeta_{6}\right)$. Accordingly we have a decomposition

$$
\mathbf{H}^{2}\left(Y_{s}, \mathbb{Q}\right)=\mathbf{M}_{1} \oplus \mathbf{M}_{2} \oplus \mathbf{M}_{3} \oplus \mathbf{M}_{6}
$$

where $\mathbf{M}_{m}$ is the summand on which $\alpha^{*}$ has order $m$. The assertion can be proven for each $\mathbf{M}_{m}$ separately. The summand $\mathbf{M}_{1}$ is the motive $i^{*} \mathbf{H}^{2}\left(\mathbb{P}^{3}\right) \cong \mathbf{1}(-1)$ where $i$ is the embedding $Y_{s} \hookrightarrow \mathbb{P}^{3}$. The summand $\mathbf{M}_{2}$ is the $\mathbf{H}^{2}$ of the K3 surface of degree 2 that is obtained as the double cover of $\mathbb{P}^{2}$ branched over the sextic curve given by $s=0$. As discussed in Section 4.4, the assertion is true for this summand. For the remaining two summands $\mathbf{M}_{3}$ and $\mathbf{M}_{6}$, we argue as in the case $d=5$. In both cases we find an abelian variety $B$ and an abelian scheme $a: A \rightarrow S$, both with an action of the cyclotomic field $E=\mathbb{Q}\left(\zeta_{m}\right)$, for $m=3$, respectively $m=6$, such that $\mathbf{M}_{m} \cong \mathbf{H}^{1}\left(A_{s}\right) \otimes_{E} \mathbf{H}^{1}(B)$, and one gets the Tate conjecture as an application of Faltings's theorem. In both cases $B$ is the elliptic curve given by $y^{2}=x^{3}-1$. The reader will have no trouble filling in the details.

Remark 4.5.3. For $d \geq 7$ the above method breaks down. The reason is that it is no longer possible to describe $\mathrm{H}^{2}\left(Y_{s}\right)$ as a "twist" of the $\mathrm{H}^{1}$ of an abelian variety. For 
instance, if we take $d=7$ and consider surfaces given by $s\left(T_{0}, T_{1}, T_{2}\right)+T_{3}^{7}=0$ then for multiplicities of the action of $\alpha^{*}$ on the holomorphic differentials we find

\begin{tabular}{c|c|c|c|c|c|c|}
$j$ & $\overline{1}$ & $\overline{2}$ & $\overline{3}$ & $\overline{4}$ & $\overline{5}$ & $\overline{6}$ \\
\hline$h^{2,0}(j)$ & 10 & 6 & 3 & 1 & 0 & 0
\end{tabular}

The obstruction to using the same method as above lies in the fact that there is a pair of complex conjugate embeddings (namely $\{\overline{3}, \overline{4}\}$ ) that both occur in the $\mathrm{H}^{2,0}$. (In [101], the authors erroneously claim that for $d=7$ one can still find a "half twist".)

\section{Acknowledgment}

I thank Johan Commelin for some helpful discussions on the topics of this paper and for his comments on a previous version.

\section{References}

[1] S. Abdulali, Algebraic cycles in families of abelian varieties. Canad. J. Math. 46 (1994), no. $6,1121-1134$.

[2] Y. André, Une remarque à propos des cycles de Hodge de type CM. In: Sém. de Théorie des Nombres, Paris, 1989-90, pp. 1-7. Progr. Math. 102, Birkhäuser, Boston, 1992.

[3] Y. André, Mumford-Tate groups of mixed Hodge structures and the theorem of the fixed part. Compositio Math. 82 (1992), no. 1, 1-24.

[4] Y. André, On the Shafarevich and Tate conjectures for hyperkähler varieties. Math. Ann. 305 (1996), no. 2, 205-248.

[5] Y. André, Pour une théorie inconditionnelle des motifs. Inst. Hautes Études Sci. Publ. Math. No. 83 (1996), 5-49.

[6] Y. André, Une introduction aux motifs (motifs purs, motifs mixtes, périodes). Panoramas et Synthèses, 17. Société Mathématique de France, Paris, 2004.

[7] Y. André, Déformation et spécialisation de cycles motivés. J. Inst. Math. Jussieu 5 (2006), no. 4, 563-603.

[8] D. Arapura, An abelian category of motivic sheaves. Adv. Math. 233 (2013), 135-195.

[9] G. Banaszak, W. Gajda, P. Krasoń, On the image of $\ell$-adic Galois representations for abelian varieties of type I and II. Doc. Math. 2006, Extra Vol., 35-75.

[10] G. Banaszak, W. Gajda, P. Krason, On the image of Galois $\ell$-adic representations for abelian varieties of type III. Tohoku Math. J. (2) 62 (2010), no. 2, 163-189.

[11] A. Beilinson, J. Bernstein, P. Deligne, Faisceaux pervers. In: Analysis and topology on singular spaces, I (Luminy, 1981), 5-171. Astérisque, 100, Soc. Math. France, Paris, 1982.

[12] B. Bhatt, P. Scholze, The pro-étale topology for schemes. Astérisque No. 369 (2015), 99-201.

[13] D. Blasius, A p-adic property of Hodge classes on abelian varieties. In: Motives (Seattle, WA, 1991), 293-308. Proc. Sympos. Pure Math., 55, Part 2, Amer. Math. Soc., Providence, RI, 1994. 
[14] F. Bogomolov, Sur l'algébricité des représentations $\ell$-adiques. C. R. Acad. Sci. Paris Sér. A-B 290 (1980), no. 15, A701-A703.

[15] M. Borovoi, The action of the Galois group on the rational cohomology classes of type $(p, p)$ of abelian varieties. (Russian) Mat. Sb. (N.S.) 94(136) (1974), 649-652, 656.

[16] N. Bourbaki, Groupes et algèbres de Lie, Chap. 7 et 8 (Nouveau tirage). Éléments de mathématique, Masson, Paris, 1990.

[17] J-L. Brylinski, "1-motifs" et formes automorphes (théorie arithmétique des domaines de Siegel). In: Conference on automorphic theory (Dijon, 1981), 43-106. Publ. Math. Univ. Paris VII, 15, Univ. Paris VII, Paris, 1983.

[18] A. Cadoret, Motivated cycles under specialization. In: Geometric and differential Galois theories, 25-55. Sémin. Congr., 27, Soc. Math. France, Paris, 2013.

[19] A. Cadoret, On $\ell$-independency in families of motivic $\ell$-adic representations. Manuscripta Math. 147 (2015), no. 3-4, 381-398.

[20] A. Cadoret, A. Kret, Galois-generic points on Shimura varieties. Algebra Number Theory 10 (2016), no. 9, 1893-1934.

[21] A. Cadoret, B. Moonen, Integral and adelic aspects of the Mumford-Tate conjecture. Preprint, https://arxiv.org/abs/1508.06426.

[22] A. Cadoret, A. Tamagawa, A uniform open image theorem for $\ell$-adic representations, I. Duke Math. J. 161 (2012), no. 13, 2605-2634.

[23] A. Cadoret, A. Tamagawa, A uniform open image theorem for $\ell$-adic representations, II. Duke Math. J. 162 (2013), no. 12, 2301-2344.

[24] E. Cattani, P. Deligne, A. Kaplan, On the locus of Hodge classes. J. Amer. Math. Soc. 8 (1995), no. 2, 483-506.

[25] F. Charles, C. Schnell, Notes on absolute Hodge classes. In: Hodge theory, 469-530, Math. Notes, 49, Princeton Univ. Press, Princeton, NJ, 2014.

[26] W. Chi, $\ell$-adic and $\lambda$-adic representations associated to abelian varieties defined over number fields. Amer. J. Math. 114 (1992), no. 2, 315-353.

[27] J. Commelin, On $\ell$-adic compatibility for abelian motives \& the Mumford-Tate conjecture for products of K3 surfaces. PhD thesis, Radboud University Nijmegen, 2017.

[28] J. Commelin, On compatibility of the $\ell$-adic realisations of an abelian motive. Preprint, https://arxiv.org/abs/1706.09444.

[29] A. de Jong, Homomorphisms of Barsotti-Tate groups and crystals in positive characteristic. Invent. Math. 134 (1998), no. 2, 301-333.

[30] P. Deligne, Équations différentielles à points singuliers réguliers. Lecture Notes in Mathematics, 163. Springer-Verlag, Berlin-New York, 1970.

[31] P. Deligne, Théorie de Hodge. II. Inst. Hautes Études Sci. Publ. Math. No. 40 (1971), $5-57$.

[32] P. Deligne, La conjecture de Weil pour les surfaces K3. Invent. Math. 15 (1972), 206226.

[33] P. Deligne, Variétés de Shimura: interprétation modulaire, et techniques de construction de modèles canoniques. In: Automorphic forms, representations and L-functions. Proc. Sympos. Pure Math. 33, Amer. Math. Soc., Providence, R.I., 1979; Part 2, pp. $247-289$. 
[34] P. Deligne, Valeurs de fonctions $L$ et périodes d'intégrales. In: Automorphic forms, representations and L-functions. Proc. Sympos. Pure Math. 33, Amer. Math. Soc., Providence, R.I., 1979; Part 2, pp. 313-346.

[35] P. Deligne, Hodge cycles on abelian varieties. (Notes by J. Milne.) In: P. Deligne, et al., Hodge cycles, motives, and Shimura varieties. Lecture Notes in Mathematics, 900. Springer-Verlag, Berlin-New York, 1982.

[36] P. Deligne, La conjecture de Weil. II. Inst. Hautes Études Sci. Publ. Math. No. 52 (1980), 137-252.

[37] P. Deligne, J. Milne, Tannakian categories. In: P. Deligne, et al., Hodge cycles, motives, and Shimura varieties. Lecture Notes in Mathematics, 900. Springer-Verlag, Berlin-New York, 1982.

[38] G. Faltings, Complements to Mordell. In: Rational points (G. Faltings, G. Wüstholz, eds.). Aspects of Math. E6, Friedr. Vieweg \& Sohn, Braunschweig, 1984.

[39] A. Grothendieck, Revêtements étales et groupe fondamental (SGA1). Séminaire de géométrie algébrique du Bois Marie 1960-61. Updated and annotated reprint of the 1971 original. Documents Math., 3. Société Mathématique de France, Paris, 2003.

[40] A. Grothendieck, On the de Rham cohomology of algebraic varieties. Inst. Hautes Études Sci. Publ. Math. No. 29 (1966), 95-103.

[41] F. Hazama, Algebraic cycles on certain abelian varieties and powers of special surfaces. J. Fac. Sci. Univ. Tokyo Sect. IA Math. 31 (1985), no. 3, 487-520.

[42] F. Hazama, Algebraic cycles on nonsimple abelian varieties. Duke Math. J. 58 (1989), no. $1,31-37$.

[43] M. Hindry, N. Ratazzi, Torsion pour les variétés abéliennes de type I et II. Algebra Number Theory 10 (2016), no. 9, 1845-1891.

[44] A. Huber, S. Müller-Stach, Periods and Nori motives. Ergebnisse der Mathematik und ihrer Grenzgebiete. 3. Folge, 65. Springer, Cham, 2017.

[45] C. Hui, Specialization of monodromy group and $\ell$-independence. C. R. Math. Acad. Sci. Paris 350 (2012), no. 1-2, 5-7.

[46] L. Illusie, Cohomologie de de Rham et cohomologie étale $p$-adique (d'après G. Faltings, J.-M. Fontaine et al.). Séminaire Bourbaki, Vol. 1989/90. Astérisque No. 189-190 (1990), Exp. No. 726, 325-374.

[47] U. Jannsen, Mixed motives and algebraic $K$-theory. Lecture Notes in Mathematics, 1400. Springer-Verlag, Berlin, 1990.

[48] U. Jannsen, Weights in arithmetic geometry. Jpn. J. Math. 5 (2010), no. 1, 73-102.

[49] P. Jossen, On the relation between Galois groups and Motivic Galois groups. Preprint, 2016, available at http://www. jossenpeter.ch/Maths.htm.

[50] J. Lewis, A survey of the Hodge conjecture (Second edition). CRM Monograph Series, 10. American Mathematical Society, Providence, RI, 1999.

[51] M. Larsen, R. Pink, On $\ell$-independence of algebraic monodromy groups in compatible systems of representations. Invent. Math. 107 (1992), no. 3, 603-636.

[52] M. Larsen, R. Pink, Abelian varieties, $\ell$-adic representations, and $\ell$-independence. Math. Ann. 302 (1995), no. 3, 561-579.

[53] A. Laskar, $\ell$-independence for a system of motivic representations. Manuscripta Math. 145 (2014), no. 1-2, 125-142. 
[54] D. Lombardo, On the $\ell$-adic Galois representations attached to nonsimple abelian varieties. Ann. Inst. Fourier (Grenoble) 66 (2016), no. 3, 1217-1245.

[55] B. Moonen, On the Tate and Mumford-Tate conjectures in codimension 1 for varieties with $h^{2,0}=1$. Duke Math. J. 166 (2017), no. 4, 739-799.

[56] B. Moonen, The Deligne-Mostow List and Special Families of Surfaces. Int. Math. Res. Not. Vol. 2017, 33pp. https://doi.org/10.1093/imrn/rnx055.

[57] B. Moonen, A remark on the Tate conjecture. Preprint, https://arxiv.org/abs/ 1709.04489.

[58] B. Moonen, Yu. Zarhin, Hodge classes and Tate classes on simple abelian fourfolds. Duke Math. J. 77 (1995), no. 3, 553-581.

[59] B. Moonen, Yu. Zarhin, Hodge classes on abelian varieties of low dimension. Math. Ann. 315 (1999), no. 4, 711-733.

[60] D. Mumford, Families of abelian varieties. In: Algebraic Groups and Discontinuous Subgroups, Proc. Sympos. Pure Math., Boulder, Colo., 1965, pp. 347-351, Amer. Math. Soc., Providence, R.I., 1966.

[61] D. Mumford, A note of Shimura's paper "Discontinuous groups and abelian varieties". Math. Ann. 181 (1969), 345-351.

[62] D. Mumford, Abelian varieties. Tata Institute of Fundamental Research Studies in Math. 5. Oxford University Press, Oxford, 1970.

[63] V.K. Murty, Algebraic cycles on abelian varieties. Duke Math. J. 50 (1983), no. 2, $487-504$.

[64] V.K. Murty, Exceptional Hodge classes on certain abelian varieties. Math. Ann. 268 (1984), no. 2, 197-206.

[65] S. Pepin Lehalleur, Subgroups of maximal rank of reductive groups. In: Autour des schémas en groupes. Vol. III, 147-172, Panor. Synthèses, 47, Soc. Math. France, Paris, 2015.

[66] C. Peters, J. Steenbrink, Mixed Hodge structures. Ergebnisse der Mathematik und ihrer Grenzgebiete. 3. Folge, 52. Springer-Verlag, Berlin, 2008.

[67] R. Pink, $\ell$-adic algebraic monodromy groups, cocharacters, and the Mumford-Tate conjecture. J. reine angew. Math. 495 (1998), 187-237.

[68] H. Pohlmann, Algebraic cycles on abelian varieties of complex multiplication type. Ann. of Math. (2) 88 (1968), 161-180.

[69] J. Ramón Marí, On the Hodge conjecture for products of certain surfaces. Collect. Math. 59 (2008), no. 1, 1-26.

[70] K. Ribet, Hodge classes on certain types of abelian varieties. Amer. J. Math. 105 (1983), no. 2, 523-538.

[71] W. Schmid, Variation of Hodge structure: the singularities of the period mapping. Invent. Math. 22 (1973), 211-319.

[72] C. Schoen, Hodge classes on self-products of a variety with an automorphism. Compositio Math. 65 (1988), no. 1, 3-32.

[73] C. Schoen, Varieties dominated by product varieties. Internat. J. Math. 7 (1996), no. 4, 541-571.

[74] C. Schoen, Addendum to [72]. Compositio Math. 114 (1998), no. 3, 329-336. 
[75] J-P. Serre, Sur les groupes de congruence des variétés abéliennes. Izv. Akad. Nauk SSSR Ser. Mat. 281964 3-20.

[76] J-P. Serre, Résumé des cours de 1965-1966. In: CEuvres, Volume II, number 71. Springer-Verlag, Berlin, 1986.

[77] J-P. Serre, Abelian $\ell$-adic representations and elliptic curves. McGill University lecture notes. Benjamin, Inc., New York-Amsterdam, 1968.

[78] J-P. Serre, Propriétés galoisiennes des points d'ordre fini des courbes elliptiques. Invent. Math. 15 (1972), no. 4, 259-331.

[79] J-P. Serre, Représentations $\ell$-adiques. In: Algebraic number theory (Kyoto Internat. Sympos., Res. Inst. Math. Sci., Univ. Kyoto, Kyoto, 1976), pp. 177-193. Japan Soc. Promotion Sci., Tokyo, 1977.

[80] J-P. Serre, Groupes algébriques associés aux modules de Hodge-Tate. In: Journées de Géom. Algébrique de Rennes. Vol. III, pp. 155-188. Astérisque, 65, Soc. Math. France, Paris, 1979.

[81] J-P. Serre, Lettres à Ken Ribet du 1/1/1981 et du 29/1/1981. In: CEuvres, Volume IV, number 133. Springer-Verlag, Berlin, 2000.

[82] J-P. Serre, Lettre à John Tate du 2/1/1985. In: Correspondance Serre-Tate. Vol. II. Documents Mathématiques, 14. Soc. Math. France, Paris, 2015.

[83] J-P. Serre, Résumé des cours de 1984-1985. In: Euvres, Volume IV, number 135. Springer-Verlag, Berlin, 2000.

[84] J-P. Serre, Propriétés conjecturales des groupes de Galois motiviques et des représentations $\ell$-adiques. In: Motives (Seattle, WA, 1991), 377-400, Proc. Sympos. Pure Math., 55, Part 1, Amer. Math. Soc., Providence, RI, 1994.

[85] J-P. Serre, Lectures on the Mordell-Weil theorem. Third edition. Aspects of Mathematics. Friedr. Vieweg \& Sohn, Braunschweig, 1997.

[86] T. Shioda, The Hodge conjecture for Fermat varieties. Math. Ann. 245 (1979), no. 2, $175-184$.

[87] T. Shioda, What is known about the Hodge conjecture? In: Algebraic varieties and analytic varieties (Tokyo, 1981), 55-68. Adv. Stud. Pure Math. 1, North-Holland, Amsterdam-New York, 1983.

[88] S. Tankeev, Algebraic cycles on abelian varieties. II. Izv. Akad. Nauk SSSR Ser. Mat. 43 (1979), no. 2, 418-429.

[89] S. Tankeev, Algebraic cycles on surfaces and abelian varieties. Izv. Akad. Nauk SSSR Ser. Mat. 45 (1981), no. 2, 398-434, 463-464.

[90] S. Tankeev, Cycles on simple abelian varieties of prime dimension. Izv. Akad. Nauk SSSR Ser. Mat. 46 (1982), no. 1, 155-170, 192.

[91] S. Tankeev, Cycles on simple abelian varieties of prime dimension over number fields. Izv. Akad. Nauk SSSR Ser. Mat. 51 (1987), no. 6, 1214-1227, 1358; translation in Math. USSR-Izv. 31 (1988), no. 3, 527-540.

[92] S. Tankeev, Surfaces of K3 type over number fields and the Mumford-Tate conjecture. Izv. Akad. Nauk SSSR Ser. Mat. 54 (1990), no. 4, 846-861; translation in Math. USSRIzv. 37 (1991), no. 1, 191-208. 
[93] S. Tankeev, Surfaces of K3 type over number fields and the Mumford-Tate conjecture. II. Izv. Ross. Akad. Nauk Ser. Mat. 59 (1995), no. 3, 179-206; translation in Izv. Math. 59 (1995), no. 3, 619-646.

[94] S. Tankeev, On the Mumford-Tate conjecture for abelian varieties. Algebraic geometry, 4. J. Math. Sci. 81 (1996), no. 3, 2719-2737.

[95] J. Tate, Endomorphisms of abelian varieties over finite fields. Invent. Math. 2 (1966), $134-144$.

[96] J. Tate, Conjectures on algebraic cycles in $\ell$-adic cohomology. In: Motives (Seattle, WA, 1991), 71-83. Proc. Sympos. Pure Math., 55, Part 1, American Mathematical Society, Providence, RI, 1994.

[97] R. Taylor, Galois representations. Ann. Fac. Sci. Toulouse Math. (6) 13 (2004), no. 1, 73-119.

[98] E. Ullmo, A. Yafaev, Mumford-Tate and generalised Shafarevich conjectures. Ann. Math. Qué. 37 (2013), no. 2, 255-284.

[99] B. van Geemen, Theta functions and cycles on some abelian fourfolds. Math. Z. 221 (1996), no. 4, 617-631.

[100] B. van Geemen, Half twists of Hodge structures of CM-type. J. Math. Soc. Japan 53 (2001), no. 4, 813-833.

[101] B. van Geemen, E. Izadi, Half twists and the cohomology of hypersurfaces. Math. Z. 242 (2002), no. 2, 279-301.

[102] A. Vasiu, Some cases of the Mumford-Tate conjecture and Shimura varieties. Indiana Univ. Math. J. 57 (2008), no. 1, 1-75.

[103] C. Voisin, Some aspects of the Hodge conjecture. Jpn. J. Math. 2 (2007), no. 2, 261296.

[104] C. Voisin, Hodge loci and absolute Hodge classes. Compos. Math. 143 (2007), no. 4, 945-958.

[105] C. Voisin, Hodge theory and complex algebraic geometry, vols. I and II. Cambridge Studies in Advanced Mathematics, vols. 76, 77. Cambridge University Press, Cambridge, 2002-03.

[106] C. Voisin, The Hodge conjecture. Open problems in mathematics, 521-543, Springer, 2016.

[107] A. Weil, Abelian varieties and the Hodge ring. Collected papers, Vol. III, [1977c], 421-429.

[108] J-P. Wintenberger, Théorème de comparaison $p$-adique pour les schémas abéliens. I. Construction de l'accouplement de périodes. In: Périodes p-adiques (Bures-sur-Yvette, 1988). Astérisque No. 223 (1994), 349-397.

[109] C. Yu, A note on the Mumford-Tate conjecture for CM abelian varieties. Taiwanese J. Math. 19 (2015), no. 4, 1073-1084.

[110] Yu. Zarhin, Endomorphisms of Abelian varieties over fields of finite characteristic. Izv. Akad. Nauk SSSR Ser. Mat. 39 (1975), no. 2, 272-277, 471.

[111] Yu. Zarhin, Abelian varieties in characteristic p. Mat. Zametki 19 (1976), no. 3, 393400. 
[112] Yu. Zarhin, Weights of simple Lie algebras in the cohomology of algebraic varieties. Izv. Akad. Nauk SSSR Ser. Mat. 48 (1984), no. 2, 264-304. (English translation: Math. USSR-Izv. 24 (1985), no. 2, 245282.)

Ben Moonen

Radboud University

IMAPP

PO Box 9010

6500GL Nijmegen

The Netherlands

e-mail: b.moonen@science.ru.nl

Lecture given at the Seminario Matematico e Fisico di Milano on April 6, 2016

Received: September 1, 2017.

Accepted: October 2, 2017.

Open Access This article is distributed under the terms of the Creative Commons Attribution 4.0 International License (http://creativecommons.org/licenses/by/4.0/), which permits unrestricted use, distribution, and reproduction in any medium, provided you give appropriate credit to the original author(s) and the source, provide a link to the Creative Commons license, and indicate if changes were made. 